\title{
40 ans d'histoire de la « lecture littéraire » au secondaire à partir de la revue Pratiques
}

A 40 Years History of the "Literary Reading" at Secondary Schools in the Journal Pratiques

\section{André Petitjean}

\section{(2) OpenEdition}

\section{Journals}

Édition électronique

URL : http://journals.openedition.org/pratiques/2155

DOI : 10.4000/pratiques. 2155

ISSN : 2425-2042

Éditeur

Centre de recherche sur les médiations (CREM)

Édition imprimée

Date de publication : 5 juin 2014

Référence électronique

André Petitjean, « 40 ans d'histoire de la « lecture littéraire » au secondaire à partir de la revue Pratiques », Pratiques [En ligne], 161-162 | 2014, mis en ligne le 05 juin 2014, consulté le 14 novembre 2019. URL : http://journals.openedition.org/pratiques/2155; DOI : 10.4000/pratiques. 2155

Ce document a été généré automatiquement le 14 novembre 2019.

(c) Tous droits réservés 


\title{
40 ans d'histoire de la « lecture littéraire » au secondaire à partir de la revue Pratiques
}

\author{
A 40 Years History of the "Literary Reading" at Secondary Schools in the Journal \\ Pratiques
}

André Petitjean

Je remercie Bertand Daunay, Jean-Louis Dufays et Yves Reuter pour leur lecture attentive de cet article.

\section{Introduction}

Dans le cadre de la $40^{e}$ année de la création de Pratiques et en attendant le colloque international intitulé «Pratiques et l'enseignement du français : 40 ans de recherches », qui aura lieu à Metz les 8, 9 et 10 avril 2015, je voudrais analyser les travaux didactiques concernant ce qu'il est convenu d'appeler la « lecture littéraire ». Pour ce faire, en écho au numéro 46 de Repères (Daunay \& Grossmann, 2012) consacré, à partir des revues, à 20 ans de recherches en didactique du français au niveau du primaire, j'ai privilégié la revue Pratiques, riche en études dont les objets et les méthodes divergent mais qui toutes apportent un éclairage particulier sur les théories littéraires et sur l'enseignement de la littérature. Ce qui signifie que je donnerai la priorité aux recherches relevant de la didactique du français langue maternelle (DFLM) et ne ferai qu'allusion à leurs homologues en didactique du français langue étrangère (FLE). Par ailleurs, je limiterai mes investigations à l'enseignement tel qu'il est organisé en France au détriment des pratiques en vigueur dans l'espace des pays francophones (voir, pour la Belgique, Dieu, Druart \& Renard, 1995 ; Dufays \& Rosier, 1999 ; Dufays, 2010 ; pour le Québec, Melançon et al., 1993 ; Lebrun \& Roy, 1999 et, pour la Suisse, Cordonier, 1997). J'ajoute qu'en complément au présent numéro, j'ai mis l'accent sur les problèmes liés à la lecture et parlerai peu du rôle de l'écriture comme moyen de donner le « goût de la 
littérature». Je précise, aussi, que ma réflexion portera essentiellement sur la didactique de la lecture au niveau du secondaire et que je n'entrerai pas dans le débat de l'opportunité ou non de l'introduction, depuis 2002, de l'enseignement de la littérature dans le premier degré (voir les numéros 37, 40 et 46 de Repères) et des dangers que signale J.-L. Chiss (2012) de la mise en avant systématique du " paradigme interprétatif » dans les classes du cycle 3, sur lequel le texte officiel de 2008 revient en partie.

2 Le fait que la littérature (je ne parle pas de celle qui s'écrit et se lit mais de celle qui s'enseigne) fasse problème n'est pas vraiment nouveau. C'est pourquoi, dans une première partie («Enseignement de la littérature au collège et au lycée ») et plus particulièrement dans sa première sous-partie ("Un enseignement problématique »), je commencerai par expliciter les raisons pour lesquelles cette discipline est en état de crise quasi structurel, comme l'ont bien analysé B. Daunay $(2006,2007)$ ou les contributeurs du numéro 118 de la revue Études de linguistique appliquée, dont M. Jey (2000).

Dans une seconde sous-partie («Brève histoire de l'enseignement de la littérature depuis sa création»), je ferai une rapide rétrospective de l'enseignement de la littérature depuis qu'à la fin du xix siècle il s'est progressivement substitué à la rhétorique, et montrerai que l'état de crise de cet enseignement a connu des variations conjoncturelles. Ce dont rendent compte les études d'A. Chervel (1998), M. Jey (1998), V. Houdart-Mérot (1998) et J.-F. Massol (2004) ou les observations de P. Aron et A. Viala (2005).

4 Dans la seconde partie ("La "lecture littéraire" : aspects théoriques et didactiques »), qui forme l'objet principal de ma réflexion, j'examinerai les apports théoriques de différents champs de référence et les réponses didactiques apportées par la revue Pratiques aux problèmes rencontrés depuis les années 70 en matière de «lecture littéraire ». Ce faisant, il m'arrivera de situer les articles publiés dans la revue dans le contexte des recherches en didactique de la littérature, qu'elles concernent le niveau du primaire (Tauveron, 2002 ; Reuter, 1996a) et surtout celui du secondaire (Dufays, 1997, 2001, 2006, 2011 ; Dufays et al., 1996a, 1996b ; Reuter, 1990, 2000 ; Canvat, 1992, 1999 ; Daunay, 1997, 1999; Rouxel, 1996 ; Chevalard-Mandroux \& Tauveron, 1998; Langlade, 2004; Langlade \& Rouxel, 2004) ou les deux ordres d'enseignement (Demougin \& Massol, 1999 ; Brillant \& Massol, 2005), voire les trois, incluant l'université (Butlen \& Houdart-Merot, 2009).

5 Dans une troisième partie, je mettrai en perspective la «lecture littéraire » tant par rapport à l'enseignement/apprentissage de l'écriture que par rapport à des théories de référence mésestimées (je pense aux travaux psychocognitifs) ou à des problématiques (textes et histoire; textes et valeurs) qui la prolongent.

6 Dans une quatrième partie, j'évoquerai l'état actuel des théories de référence ainsi que des orientations de recherches en didactique et mentionnerai quelques propositions de recherches en cours dans Pratiques.

7 Dans la conclusion, j'interrogerai la notion de compétence, qui depuis quelques années fait florès dans notre système éducatif (Allal, 1999 ; Colles et al., 2001 ; Dolz \& Ollagnier, 2002 ; Dufays, 2011) et mesurerai quelle est sa pertinence pour l'enseignement de la littérature. 


\section{Enseignement de la littérature au collège et au lycée}

\subsection{Un enseignement problématique}

8 Si par définition le mot " crise » signifie " rupture d'équilibre », ce qui présuppose un fond de stabilité, on peut s'interroger sur le statut de l'enseignement de la littérature quand on constate que l'état de crise lui semble consubstantiel. Je me réfère à $G$. Lanson (1909) qui parle de la «crise des méthodes dans l'enseignement du français» au moment où l'enseignement de la littérature s'est institué à la charnière des $\mathrm{XIX}^{\mathrm{e}} \mathrm{et} \mathrm{xx}^{\mathrm{e}}$ siècles et à P. Kuentz (1972) qui évoque la «crise de l'enseignement de la littérature » quand cette "configuration historique de pratiques discursives" a commencé à se déliter dans les années 60-70 du siècle dernier. Plus récemment, E. Fraisse et V. Houdart-Merot (2004) déclarent que « chacun s'accorde à reconnaitre que les études littéraires sont en crise", tandis qu'A. Rouxel (2005) parle de "crise de l'identité disciplinaire" que connait l'enseignement du français au collège. Au-delà des spécificités conjoncturelles de ces «crises» (voir infra), il semble bien que l'enseignement de la littérature est en état de crise permanent. Comment imaginer qu'il puisse en être autrement dans la mesure où il se voit attribuer des finalités et des objectifs pour lesquels les consensus ne sont pas évidents à trouver, à savoir :

- l'appétence pour la lecture des textes littéraires ;

- la participation à l'acquisition de compétences langagières ;

- le développement du goût d'écrire et de la compétence scripturale ;

- le développement du sens esthétique ;

- la possibilité d'un enrichissement existentiel par la découverte de situations et de comportements humains ;

- le développement de l'imaginaire et de la créativité ;

- l'exercice du jugement et l'aiguisement du sens critique ;

- la connaissance de l'histoire ;

- l'acquisition d'une culture générale.

Ce que constatait Y. Reuter (1999 : 197) qui écrit :

«La variété des objectifs et finalités assignés à l'enseignement-apprentissage de la littérature est aussi impressionnante : développer l'esprit d'analyse, développer les compétences linguistiques, développer les compétences en lecture et en écriture, développer les savoirs en littérature, développer le bagage culturel de l'élève, développer son esprit critique, lui permettre de s'approprier un patrimoine, développer son sens de l'esthétique et sa sensibilité, lui faire prendre du plaisir, participer à la formation de sa sensibilité... »

Ces finalités et ces objectifs se retrouvent tout au long des Instructions officielles (IO) de l'enseignement secondaire - voir, par exemple, Enjeux 43/44, intitulé «Littérature : les programmes francophones" ou l'ouvrage Histoire de l'enseignement du français et textes officiels (Petitjean \& Privat, 1998) - mais aussi dans un document tel Le Cadre européen commun de référence pour les langues (2008: 82) qui sert, depuis les années 90, de recommandations à l'usage de l'apprentissage/enseignement des langues vivantes :

«Les littératures nationale et étrangère apportent une contribution majeure au patrimoine culturel européen que le Conseil de l'Europe voit comme une ressource commune appréciable qu'il faut protéger et développer. Les études littéraires ont de nombreuses finalités éducatives, intellectuelles, morales et affectives, linguistiques et culturelles et pas seulement esthétiques. » 
11 Ces finalités ne sont pas de même nature et impliquent des tensions potentielles telles la nécessaire adaptation sociale de l'individu et sa faculté d'émancipation, l'affirmation de soi et le respect des différences ; le développement de l'imaginaire et l'exercice de la raison... Autant de facteurs qui font que l'enseignement de la littérature est, au sein de la discipline « français », une matière particulièrement axiologisée.

12 Il en résulte, que l'on se place au niveau des décideurs responsables des programmes, des auteurs de manuels ou de chaque enseignant, des choix permanents à effectuer. C'est ainsi, comme le montrent A. Chervel (1986) M. Jey (1998), V. Houdart-Merot (2004a) et les numéros 155 et 172 du Français aujourd'hui et 32 d'Enjeux, que la volonté de s'adapter au public scolarisé tout en défendant le principe de la conservation d'un patrimoine sous la forme d'un panthéon d'auteurs se traduit par des conflits concernant l'élargissement ou non du corpus des œuvres de référence. Autre exemple, il est corollaire au précédent: l'introduction de la littérature de jeunesse dans le premier cycle de l'enseignement secondaire fait l'objet de polémiques incessantes. Peut-on attribuer aux œuvres qui appartiennent au champ de la littérature de jeunesse la reconnaissance et l'autorité des œuvres labellisées comme littéraires et, si oui, faut-il imposer un canon? Je renvoie à l'abondante littérature critique sur le sujet, entre autres, aux travaux de B. Friot (1996) et M. Butlen (2004) qui montrent combien les textes officiels, en dehors des IO de 2000, n'intègrent qu'avec réticence la littérature de jeunesse en la réservant, au mieux, au primaire et au premier cycle du collège, pire, en la reléguant hors du champ scolaire, comme l'imposent les textes officiels pour le collège de 2008. Inversement, l'ouvrage de P.-M. Beaude, J.-M. Privat et A. Petitjean (1996), les numéros 52 d'Études de linguistique appliquée, 149 du Français aujourd'hui, et 47 et 88 de Pratiques), ainsi que les articles de C. Tauveron (2001) et A. Armand (2001), rendent compte du potentiel culturel et didactique de cette littérature.

À ces finalités s'ajoutent des objectifs plus spécifiques que l'on fixe à l'enseignement de la littérature, du type maitrise de la langue ou apprentissage du lire et de l'écrire. Il s'ensuit que le texte littéraire en situation scolaire peut être appréhendé à la fois comme un instrument un document et un monument.

14 Un "instrument» dans la mesure où il est tout à fait loisible de se servir d'extraits d'œuvres littéraires, romanesques ou théâtrales - sans souscrire à leur usage normatif à des fins grammaticales ou moralisatrices - pour travailler, par exemple, sur les temps verbaux (Adam, 1976), la progression thématique (Adam, 1977; Combettes, 1993), le point de vue (Perrin, 2004; Rabatel, 2003) ou sur les différentes formes de discours rapporté (Combettes, 1990).

15 Il est possible aussi d'aborder les textes littéraires comme des documents au sens où ils représentent des sources de savoirs, même s'ils accomplissent leur «fonction spéculative " (Macherey, 2010) et "travaillent des schèmes de pensée » sur un mode autre que celle des textes scientifiques, puisque les énoncés de fictions ne sont «ni vrais ni faux » et n'ont pas à se soumettre à l'impératif de la vérité, de la neutralité axiologique et de la falsifiabilité. C'est en ce sens que R. Barthes (2002: 433) parle de ces archives particulières qu'il appelle "monuments »: «La littérature prend en charge beaucoup de savoirs [...] Si par je ne sais quel excès de socialisme ou de barbarie, toutes nos disciplines devaient être expulsées de l'enseignement sauf une, c'est la discipline littéraire qui devrait être sauvée, car toutes les sciences sont présentes dans le monument littéraire ». Les œuvres permettent, en effet, de comprendre les modes de pensée de leur époque car, comme l'écrit G. Lanson (1904) à propos des «grandes 
personnalités littéraires »: "Ce sont des foyers qui concentrent à un moment des rayons émanés de la collectivité, et qui les renvoient ensuite, diversement combinés et modifiés, à la collectivité. [...] Ce que le génie individuel a [...] de plus grand, ce n'est pas la singularité qui l'isole, c'est dans cette singularité même, de ramasser en lui et de symboliser la vie collective d'une époque et d'un groupe ».

Le texte littéraire se prête aussi à des découvertes ultérieures de savoirs latents ou virtuels - ce que Y.Citton (2007) appelle «actualisation»-, que d'autres contextes épistémiques révèlent, qu'ils concernent les processus psychiques, le fonctionnement de la langue ou les rapports sociaux.

V. Jouve (2010 : 107) le souligne :

«L'auteur ne maitrisant pas tout ce qu'il investit dans son texte, certains contenus ne seront identifiés que très longtemps après la parution de l'œuvre, une fois que l'on aura acquis les outils théoriques permettant de les repérer. Auparavant, les savoirs en question seront simplement "ressentis", comme on peut éprouver l'effet d'une symphonie sans rien connaitre en musicologie. C. Eglin appréhende ainsi le texte littéraire comme une configuration signifiante en attente d'un cadre interprétatif qui l'éclaire ».

18 Il reste que le texte littéraire est avant tout considéré comme un monument, lié à un auteur et formant avec d'autres œuvres un patrimoine inscrit dans une mémoire collective. A. Compagnon (2000) le rappelle : « Toute personne qui écrit ou a écrit n'est pas un auteur, la différence étant celle du document et du monument. Les documents d'archives ont eu des rédacteurs; les monuments survivent. Seul le rédacteur dont les écrits sont reconnus comme des monuments par l'institution littéraire atteint l'autorité de l'auteur ».

19 C'est ainsi que M. Foucault (1969: 187) écrivait à propos du «monument» que, pour entendre la voix qui s'exprime à travers lui, tout un travail est nécessaire "qui transforme le document en monument [...] l'organise, le découpe, le distribue, l'ordonne, le répartit en niveaux, établit des séries, distingue ce qui est pertinent de ce qui ne l'est pas, repère des éléments, définit des unités, décrit des relations ». En fait, le partage entre «document» et "monument» dépend pour une large part d'une "décision de lecture ». Ce qu'affirmait J.-M. Schaeffer (1996) quand il distingue face à un texte une «attention scientifique » d'une «attention esthétique» et que confirme B. Vouilloux (2004: 252) :

«Les changements de focale induits par la modalité attentionnelle recoupent la partition bien connue, et déjà alléguée ici, même entre le "monument", comme objet d'enquête, et le "document" comme instrument d'enquête. La focalisation sur les aspects informationnels d'œuvres constitutivement ou conditionnellement littéraires revient à les manipuler comme des documents, tandis que la focalisation sur les propriétés littéraires d'un texte éventuellement régi par des principes autres le fait accéder au statut de monument ».

Pour ne prendre qu'un exemple, il est tout à fait possible, si j'en juge par certains articles de Pratiques, de lire Zola en considérant qu'il est l'auteur d'œuvres majeures du $\mathrm{XIX}^{\mathrm{e}}$ siècle (monument) et d'accompagner la lecture de ses romans d'un appareillage critique et didactique qui en apporte la preuve. Ce dernier prend la forme de savoirs et de connaissances d'ordre contextuel et institutionnel (Pagès, 2000), génétique (Scarpa, 2000), intertextuel (Tauveron, 2000), poétique (Masseron \& Petitjean, 1979; Duhamel \& Masseron, 1987). 
21 Il est enfin loisible de montrer combien les romans de Zola ainsi que ses dossiers génétiques (Mitterrand, 1986 ; Becker, 1986) sont autant de documents ou de monuments au sens barthésien, de type anthropologique, sociologique ou linguistique sur le XIX siècle. Un roman comme Germinal rend compte de la condition ouvrière dans les mines du Nord à l'époque de Zola et exemplifie les difficultés de faire entendre des « voix populaires » dans un texte écrit en français langue nationale.

22 Au total, on peut dire que, compte tenu de la multiplicité des finalités et des objectifs allouée à l'enseignement de la littérature, on comprend qu'il subisse des tensions permanentes. Avant de le démontrer, je vais me permettre une double digression en forme de complément explicatif du statut problématique de la littérature et de son enseignement.

23 La première pour constater, comme le fait T. Todorov (1978), qu'une «entité "littérature" fonctionne au niveau des relations intersubjectives et sociales " sans que l'on puisse définir de façon consensuelle le concept qui lui correspond ou encore, comme l'écrit A. Compagnon (1998: 48) : «La définition d'un terme comme littérature ne donnera jamais autre chose que l'ensemble des occurrences dans lesquelles les usagers d'une langue acceptent d'employer ce terme ».

24 Il est alors tentant, comme le fait G. Genette (1991) d'admettre que s'interroger sur la définition de la littérature est "une sotte question » et que «la vraie sagesse serait peut-être de ne pas se la poser ». Et cela d'autant plus que le terme de « littéraire », à propos des textes scolarisés, n'est finalement pas des plus judicieux dans la mesure où il qualifie plus un domaine qu'un objet, tout en référant à un mode de lecture. En effet, les textes labellisés comme étant "littéraires " sont génériquement hétérogènes (fictions, essais...), et on peut se demander ce qui permet d'intégrer dans la même classe de discours les œuvres de Saint Simon, Pascal, Beaumarchais, Balzac ou Leiris. Les réponses, car elles existent, ne sauraient être linguistiques ou sémiotiques (voir infra à propos de la « littérarité ») mais sont d'inspiration plutôt philosophique, sociologique, anthropologique, didactique ou scolaire.

D’un point de vue philosophique, P. Ricoeur (1986: 53) souligne l'enjeu existentiel des textes littéraires :

«Contrairement à la tradition du Cogito et à la prétention du sujet de se connaitre lui-même par intuition immédiate, il faut dire que nous nous comprenons que par le grand détour des signes d'humanité déposés dans les œuvres de culture. Que saurions-nous de l'amour et de la haine, des sentiments éthiques et en général, de tout ce que nous appelons le soi, si cela n'avait pas été porté au langage et articulé par la littérature?»

De même M. Merleau-Ponty (1960: 84) considère que la littérature « donne à penser » et explique que

"l'usage vivant du langage, ignoré du formalisme aussi bien que de la littérature à "sujets", est la littérature même comme recherche et acquisition. [...] Ce qui n'est pas remplaçable dans l'œuvre d'art, ce qui fait d'elle beaucoup plus qu'un moyen de plaisir: un organe de l'esprit, dont l'analogue se retrouve en toute pensée philosophique ou politique si elle est productive, c'est qu'elle contient, mieux que des idées, des matrices d'idées, qu'elle nous fournit d'emblèmes dont nous n'avons jamais fini de développer le sens, que, justement parce qu'elle s'installe et nous installe dans un monde dont nous n'avons pas la clef, elle nous apprend à voir et finalement nous donne à penser comme aucun ouvrage analytique ne peut le faire, parce que l'analyse ne trouve dans l'objet que ce que nous y avons mis. Ce qu'il y a de hasardeux dans la communication littéraire, et d'ambigu, d'irréductible à la 
thèse, dans toutes les grandes œuvres de l'art n'est pas une faiblesse provisoire, dont on pourrait espérer les affranchir, c'est le prix qu'il faut payer pour avoir une littérature, c'est-à-dire un langage conquérant, qui nous introduise à des perspectives étrangères, au lieu de nous confirmer dans les nôtres ».

Au regard de la sociologie, dans la mesure où pour P. Macherey (1970), R. Balibar (1974) ou Y. Reuter (1990), c'est la fonction qui crée l'organe et non l'inverse, le qualificatif de « littéraire " réfère moins à une propriété interne des textes qu'à une construction sociale à laquelle l'institution scolaire contribue pour une part prépondérante. C'est ainsi que R. Balibar (1974 : 44) écrit : « Est littéraire le texte reconnu comme tel, et il est reconnu comme tel précisément dans le temps, et dans la mesure où il provoque pratiquement des interprétations, des critiques, des "lectures". Ce pourquoi un texte peut très bien cesser d'être littéraire, ou de le devenir dans des conditions qui d'abord n'existaient pas. »

La dimension anthropologique de la littérature affleure dès l'instant où il s'agit de considérer les textes appelés " littéraires » comme des « discours constituants ». Ce que propose D. Maingueneau (2004:47-48), quand il écrit :

«L'expression de "discours constituant" désigne fondamentalement ces discours (littérature, philosophie, religion, mythe, science) qui se donnent comme discours d'Origine, validés par une scène d'énonciation qui s'autorise d'elle-même. [...] Les discours constituants sont en charge de ce que l'on pourrait appeler l'archéion d'une collectivité. [...] Les discours constituants sont en effet les garants de multiples genres de discours. Le journaliste aux prises avec un débat de société en appellera ainsi à l'autorité du savant, du théologien, de l'écrivain ou du philosophe, mais non l'inverse. Ils possèdent ainsi un statut singulier : zones de parole parmi d'autres et paroles qui se prétendent en surplomb de toute autre. »

En fonction de quoi, on peut justifier, d'un point de vue didactique, la place réservée à la littérature dans l'enseignement du français compte tenu de ses vertus éducatives. Ce qu'affirme J.-P. Bronckart $(2013: 22)$ :

« Nous soutiendrons que la validité éducative générale de la littérature tient à ce que celle-ci est le lieu du débat permanent sur les valeurs de tous ordres qui orientent les activités humaines, et que les citoyens en devenir que constituent les élèves doivent être aptes à entrer dans ce débat, à participer à ce travail interprétatif permanent, par lequel se constituent l'autonomie et la socialité des personnes. [...] On peut considérer ensuite que la littérature propose des situations dans lesquelles les mobiles des actions sont clarifiés ou, à tous le moins, discutés, problématisés, et que c'est en entrant dans cette production littéraire que l'humain développe, solidairement, sa socialité et sa personnalité. À ces objectifs premiers sont en outre, quasi nécessairement associés des objectifs complémentaires. D'un côté, pour autant que l'on accepte de proposer et de confronter des corpus de textes constitués des littératures universelle, nationale et régionale, cet enseignement est une occasion d'entrer dans le débat sur la diversité sociale et culturelle. D'un autre côté, les textes littéraires témoignent, dans leur structure énonciative même, de l'évolution des positionnements philosophiques à l'égard de la question du sujet, et cet enseignement est donc aussi une occasion d'entrer dans le débat philosophique, pour autant que l'on accepte de proposer et de confronter des corpus de textes qui attestent de cette évolution. Enfin, l'enseignement de la littérature est assurément aussi l'occasion d'une formation linguistique, d'une mise en évidence des possibilités multiples, voire infinies, de structuration et de restructuration des ressources de la langue, au service de l'activité communicative ».

Plus généralement, comme je l'ai montré (Petitjean, 1999), c'est l'institution scolaire qui, d'un texte officiel à l'autre, établit, à l'aide d'un « canon » variable historiquement, la frontière entre ce qui est littéraire et ce qui ne l'est pas (voir Jey, 1998, 1999; Fraisse, 
1985). Dans tous les cas, les textes sélectionnés - anthologies (Fraisse, 1997), listes officielles de "classiques » pour le baccalauréat (Veck, Robert-Lazès \& Robert, 1996), etc. - le sont dans la mesure où on leur prête une "valeur » qui elle-même dépend de critères autant fonctionnels que formels. C'est ainsi qu'au fil des textes officiels aux valeurs du Beau, du Vrai et du Bien se sont ajoutées celles de la Sensibilité, de l'Imagination et de la Littérarité.

Comme l'écrivent J.-P. Goldenstein (1983a : 4) et Y. Reuter (1995 : 70) :

«La littérature scolaire est ce qui est reconnu comme faisant partie du corpus "littérature" par l'école et conjointement le mode de consommation de ce corpus ». "Ce qui s'enseigne sous le nom de littérature et de lecture littéraire consiste d'abord, institutionnellement en la reconnaissance d'un corpus, de valeurs à lui attribuer, d'un type de relation à ce corpus et en la "naturalisation" de cette reconnaissance. En conséquence ce qui s'enseigne est à la fois une reconnaissance et une posture ".

On le voit, accorder une légitimité et une "valeur littéraire " (Lafarge, 1983) à un ensemble de textes relève de prises de décision collectives. G. Genette (1991) parle de « littérarité conditionnelle » soumises à la variation historique et à un conditionnement interprétatif (voir l'historique de l'enseignement de la littérature dont j'ai déjà parlé). Le fait que certaines œuvres bénéficient d'une "caution institutionnelle », qu'elle prenne la forme de la réputation d'un éditeur et d'une collection, du nombre de discours critiques attachés à un texte ou du volume de péritextes qui influent sur la pré-compréhension des lecteurs, est indéniable. Comme le pense B. Vouilloux (2004: 215), «à cette valorisation par présupposition (“Je m'intéresse à Balzac parce qu'il est important historiquement") se joignent les effets de la valorisation récursive, où l'attribution d'une valeur à l'objet résulte de la valorisation auto-légitimante qui s'attache au travail sur cet objet. »

31 C'est ainsi que J.-P. Goldenstein (1990) a pu montrer que l'extrait de l'annuaire téléphonique qu'A. Breton recopie quasi intégralement et présente comme un poème (PSST) en changeant de statut générique (texte utilitaire/texte artistique) entraine une modification radicale du régime de sa lecture. Ce qui confirme la justesse de la position de T. Aron (1984a), à savoir que « lire un texte comme littéraire, c'est s'attendre à ce que tout élément y fasse signe ».Témoignent aussi de la prégnance des jugements de valeur collectifs l'expérience de I. A. Richards (1929) relatée par J. Molino (1984) ou l'expérimentation de S.Fish (1980), pour ne prendre que deux exemples emblématiques. Le premier soumet à ses étudiants des poèmes pour lesquels il a enlevé toute information contextuelle (sujet, auteur, époque...) et leur demande de les commenter librement. Le second, au sortir d'un cours sur la théorie de la littérature, laisse au tableau une liste de noms de critiques à laquelle il a ajouté un cadre et un numéro de page. Au cours de la matinée, il présente aux étudiants d'un autre cours consacré à la poésie religieuse, le pseudo-poème comme étant un poème religieux datant $\mathrm{du} \mathrm{XVII}{ }^{\mathrm{e}}$ siècle et leur demande de l'interpréter. Il ressort, de l'expérience de I. A. Richards que les jugements de valeurs imprègnent profondément les lectures des étudiants et que leurs points de vue divergent quant à la sélection des faits textuels sur lesquels ils s'appuient, ou s'en dispensent, pour justifier leurs interprétations. Quant à S. Fish, son expérience tend à accréditer le fait que la situation et la consigne, sans oublier la forme du texte, induisent l'adoption d'une posture interprétative au cours de laquelle les étudiants retrouvent dans le texte les propriétés typiques du langage poétique qu'on leur a enseignées. Dans la mesure où les étudiants en question sont des 
étudiants littéraires ayant quelques années de commentaire derrière eux, ces deux expériences sont intéressantes à double titre. D'une part, elles nous rappellent que pour la lecture "ordinaire» les textes fonctionnent comme autant de «tests projectifs» qui révèlent la personnalité et la culture du lecteur, plus que l'intériorisation des normes scolaires transmises par la pratique du commentaire. D'autre part, elles confirment que le postulat de «littérarité », qui dépend pour une large part du regard subjectif du lecteur, est néanmoins surdéterminé par la " communauté interprétative " à laquelle il appartient. D'une culture à l'autre et à l'intérieur d'une même culture, ce qui change est moins le fait d'attribuer de la valeur à certains textes que les modalités mêmes (formes et contenus) de leurs modes d'interprétation.

Ce qui permet à S. Fish d'écrire :

"D'un côté, il semble qu'il n'y ait aucun fondement pour déclarer une interprétation inacceptable, mais d'un autre côté, nous nous livrons sans cesse à de telles déclarations. Cela ne constitue une impasse que si nous assumons que l'activité d'interprétation est elle-même inconditionnée. [...] En réalité, la forme de cette activité est déterminée par l'institution littéraire qui, à chaque instant donné, n'autorisera qu'un nombre fini de stratégies interprétatives ».

La seconde digression porte - et c'est un facteur supplémentaire qui explique les crises de la discipline - sur la place de l'enseignement de la littérature au sein de l'enseignement du français. Membre de la Commission Chevalier (1999-2001) puis du Groupe Technique disciplinaire responsable des Instructions Officielles pour le français au lycée (1999-2001), je mesure combien le français est une discipline qui fait l'objet de positions difficilement conciliables et soumises aux aléas des changements politiques sans que jamais l'institution ne se donne les moyens d'évaluer les réformes qu'elle engage. Il y a d'un côté ceux pour qui, comme l'argumente J.-F. Halté (1992, 1995), la matrice disciplinaire du français doit être fédérée par des «objets centraux et des objectifs décisifs » que subsume la formule "production et réception de discours oraux et écrits ». Ce qui a nécessairement pour effet sinon de réduire la place de la littérature dans l'enseignement du français, du moins d'en finaliser autrement son usage. Au cours des années 70-80, la littérature a perdu, de fait, la place hégémonique qui était la sienne, dans la mesure où le cahier des charges de la discipline a dû se modifier au sens où il lui faut désormais prendre en compte le développement des besoins langagiers (oraux et écrits) des élèves. L'espace réservé aux textes littéraires s'est aussi restreint car ils ont été concurrencés par d'autres discours, qu'ils relèvent du champ de la littérature de jeunesse, proviennent de ce que l'on appelle les "discours sociaux " (presse, images, publicités...) ou concernent d'autres pratiques artistiques (cinéma, peinture). Significatif, de ce point de vue, la vision intégrationniste de la littérature que l'on trouve dans le bilan de la DAF (Didactique et acquisition du français langue maternelle) qui recense et analyse l'ensemble des recherches sur le français langue maternelle dans les pays francophones pour la période 1970-1985 (voir Gagné, Lazure, Sprenger-Charolles \& Ropé, 1989). Comme s'en explique G. Pastiaux-Thiriat (1997), le texte littéraire ne figure pas au nombre des descripteurs du thésaurus mais est inclus dans une rubrique appelée "Textes et documents ». On y trouve " les genres littéraires (poésie, théâtre, roman), des sous-genres (littérature enfantine, roman policier, science-fiction), des documents pluri-codés (bande dessinée, roman photo), les médias (presse écrite, radio, télévision), le film, le texte publicitaire ainsi que des textes informatifs ou explicatifs (textes scientifiques, documentaires) ». S'il est aisé de trouver 
un écho dans Pratiques aux recherches concernant ces différents objets discursifs ( $n^{\circ} 18 / 19$, « Arrêts sur images »; 37, « La télé à l'école »; 47, « Littérature de jeunesse »; 54, «Mauvais genres »; 94, «Genres de la presse »), il est par contre indéniable que nous n'avons jamais délaissé l'étude de la littérature, comme on le verra, mais sur un mode autre que la sacralisation et la vénération. En revanche, il y aurait à redire sur les pratiques proposées par les manuels de collèges des années 70 qui, sous couvert d'expression et de communication, ont multiplié les références aux « textes vivants » et mis sur le même plan, par le biais du "thématisme ", des documents divers relevant de genres hétéroclites. Par la suite, la matrice disciplinaire du français a continué à évoluer et les manuels des années 90 ont progressivement intégré la perspective textuelle et discursive à la diffusion de laquelle Pratiques a fortement contribué et que les textes ministériels de 2000 ont largement officialisée.

De l'autre côté, en opposition frontale par rapport à ceux que l'on a appelé les "rénovateurs » ou les "refondateurs ", on trouve les partisans des Humanités (voir Gally, 2006). Ils estiment que la littérature et les textes qu'elle labellise comme tels doivent demeurer avec les exercices canoniques qui les accompagnent au cœur de la matrice disciplinaire. Est symptomatique, à cet égard, l'opposition qui perdure entre les statuts revendiqués de "professeur de Lettres » et d'« enseignant de français ». C'est ainsi que D. Sallenave (1995: 146) peut écrire que « c'est à la littérature que l'on devrait principalement confier le soin d'enseigner le fonctionnement du langage (car) le langage n'est qu'une application des pouvoirs de la littérature».

On reste perplexe devant une telle méconnaissance des divers usages, tant oraux qu'écrits d'une langue selon les genres de discours et surtout du fait que comme l'écrit D. Maingueneau (1986: 10), "le texte littéraire (est) un "pseudo-énoncé" qui ne communique qu'en pervertissant les contraintes de l'échange linguistique ».

Non moins sévères et intempestives sont les déclarations d'Henri Mitterand (1992), lui qui a dirigé une collection de manuels baptisés "Langue et langages ", quand il écrit que les programmes en vigueur ne font pas mention de "l'œuvre, du livre, de l'imaginaire, du rêve, de désir, de l'émotion, de la pensée, de la fiction de style, de la poésie, de génie, de l'admiration ».

C'est en voulant opérer une "refondation" sous la forme d'une synthèse entre les conceptions divergentes de la discipline, que les Instructions Officielles de 2000 pour les lycées ont provoqué l'ire des défenseurs des Humanités (voir l'analyse qu'en fait B. Daunay, 2003, 2006) sans satisfaire pour autant les partisans de la rénovation. On sait les régressions et les restaurations qui ont suivi avec le texte officiel de 2008.

Toute aussi révélatrice de ces conflits identitaires qui traversent la discipline, l'existence aujourd'hui, au sein de la communauté des didacticiens du français, de recherches en didactique de la littérature dont l'autonomisation plus ou moins relative est revendiquée par rapport à la didactique du français comme l'attestent les nombreux colloques consacrés à la didactique de la littérature, les « Rencontres des chercheurs en didactique de la littérature » aux titres significatifs (l'enseignement de la littérature, les manuels, les corpus, les valeurs, la patrimonialisation, le Texte du lecteur, les temps et les lieux de la lecture), ainsi que les thèses soutenues dont La Lettre de l'Association internationale pour la recherche en Didactique du français (l'AIRDF) rend compte régulièrement. Alors que le colloque de Lyon consacré aux « Métalangages de la classe de français » organisé sous l'égide de la DFLM (future AIRDF) et édité par R. Bouchard et J.-C. Meyer (1995) accordait une place non négligeable (contenus et fonctions des 
métalangages dans la littérature et son enseignement) aux études littéraires, il n'en va pas de même pour la journée d'étude de Poitiers (2000), intitulée "Questions d'épistémologie en didactique du français » et organisée sous le patronage de la même DFLM (voir Marquillo-Larruy, 2001). Au cours de cette rencontre, J.-L. Dufays (2001b) se plaindra à juste titre de l'absence de reconnaissance de la didactique de la littérature et souhaitera ardemment que les chercheurs en didactique apprennent à se connaitre et à travailler ensemble. Le temps a passé et l'on ne peut pas dire que ce vœu s'est réalisé. Preuve que l'institutionnel surdétermine pour une part non négligeable l'épistémologique, il est intéressant de constater un clivage disciplinaire entre ceux qui se revendiquent comme "didacticiens de la littérature » et qui sont, pour l'université française, issus essentiellement de la section 09 (Littérature) et en partie 70 (Sciences de l'éducation) alors que les promoteurs de la didactique du français appartenaient principalement aux Sciences du langage (07). C'est bien de «champ » spécifique dont parle J.-L. Dufays (2001a) à propos des recherches en didactique de la littérature dont il définit avec pertinence les contours à l'aide d'une réflexion épistémologique et méthodologique. Ce paradigme théorique ne cesse de se développer comme le montre la radiographie que font B. Daunay et J.-L. Dufays (2007) des types de recherches menées entre 2000 et 2005. Tributaires de la logique scientifique de spécialisation et de distinction, ces recherches - Y. Reuter et D. Lahanier-Reuter (2007a) le rappellent n'évitent pas toujours de prendre trop de distance par rapport aux pratiques dans les classes et menacent d'éclatement le "paradigme unifié de la discipline " (Schneuwly, 2007). Le danger est d'autant plus grand - B. Daunay (2010) s'en inquiète - que les dernières IO du primaire en France déstructurent la discipline en deux champs (" observation réfléchie de la langue » et « littérature ») et que celles du lycée de 2008 renforcent la place de la littérature au lieu de répondre aux besoins réels des élèves en matière de langue et de littératie. Pour avoir longtemps dirigé un laboratoire de recherches (le Centre d'études linguistiques des textes et des discours) réunissant sur le modèle du collectif de Pratiques, des linguistes, des littéraires et des didacticiens, je puis témoigner de l'intérêt (sans cacher les difficultés) d'un traitement pluridisciplinaire d'objets communs de recherches et m'interroge sur les enjeux et les effets de cette sectorisation disciplinaire. Je le fais d'autant plus qu'avec le recul, je mesure la responsabilité de Pratiques dans l'émiettement disciplinaire (didactique du français, de la langue, du lexique, de l'orthographe, de la lecture, de l'écriture, du récit...). Il convient donc, dans le prolongement des numéros 137/138, 145/146 et 149/50 de Pratiques, de poursuivre la réflexion sur la discipline français, les compétences langagières et culturelles qu'elle entend développer, les savoirs qu'elle transmet et sur la place de la littérature dans cette configuration. En effet, on ne manque pas d'écrits théoriques qui rendent compte de l'articulation entre la compréhension et l'interprétation dans les processus de lecture (voir infra) et sur l'inter-relation des opérations cognitives telles qu'elles sont mises en œuvre dans la lecture de tout texte. Ce qui justifie d'autant moins la démarche qui consiste à réserver le développement des capacités interprétatives des élèves à la seule approche des textes labellisés comme littéraires, et ce dès l'âge de "cinq ans ", si l'on en croit les Instructions Officielles de l'École primaire de 2002. comme je vais le montrer à l'aide d'un bref rappel historique. 


\subsection{Brève histoire de l'enseignement de la littérature depuis sa création}

Partageant avec A.Chervel (1998), M. Develay (1995) ou Y. Reuter (et al., 2007), le principe qu'une discipline scolaire peut se définir comme un ensemble composite de connaissances, de méthodes, d'activités, de tâches, de supports matériels, de modalités de contrôle qui visent des objectifs tels qu'ils sont sous-tendus par des finalités, on peut dire que deux raisons majeures expliquent qu'une discipline scolaire puisse connaitre un état de crise :

- l'obsolescence des savoirs enseignés et des méthodes ou exercices pratiqués en référence aux savoirs dits « savants 》 (crise interne de la discipline);

- l'inadaptation des contenus de la discipline au public scolarisé, à son hétérogénéité progressive et à la demande sociale d'instruction et d'éducation (crise externe).

Ce que rappelle A. Viala (2004: 10), à propos des programmes: "Tout programme d'enseignement, dans un pays comme la France où ils sont nationaux, se doit d'être à la fois réponse à une demande sociale et affirmation des exigences fondamentales de la matière concernée ».

A. Rouxel (2005: 22) tient des propos similaires quand elle réfléchit à l'identité actuelle de la discipline : «La discipline change dans ses contenus, ses objectifs et ses finalités. L'évolution diachronique est, on le sait, commandée par les transformations de la société (demande sociale et volonté politique) et par le développement des connaissances scientifiques. Nouveaux publics, nouveaux savoirs... »

Pour illustrer le phénomène de l'adaptation au nouveau public, on se référera à la " crise du français » au cours de laquelle on voit G. Lanson (1904) s'opposer aux cours d'histoire et de théories littéraires qui débouchent sur des sujets du baccalauréat du type «Vie et œuvre de Racine» ou «Faire un tableau sommaire de la littérature française au XVI ${ }^{e}$ siècle »). G. Lanson, conscient que ces cours induisent et favorisent le psittacisme, les réserve à l'université et défend la lecture des œuvres dans le but de former l'homme et le citoyen : «À vouloir persister dans notre méthode littéraire [...], on prépare le résultat qu'attestent d'innombrables compositions du baccalauréat, où de malheureux enfants dégorgent douloureusement des formules de Taine, Brunetière, Faguet dont on les a gavés en hâte et qu'ils n'ont pas digérées. »

De même, prenant leurs distances par rapport aux exercices de composition de textes de la rhétorique ("Vous êtes Jeanne d'Arc et répondez aux accusations de l'Évêque Cauchon »), F. Brunot (1895), G. Rudler (1902) et G. Lanson promeuvent ces exercices, qui deviendront canoniques, que sont l'explication de texte et la dissertation (pour une mise en perspective historique de ces exercices, voir Chervel, 2002 ; Albertini, 1987). Même s'il émet quelques réserves par rapport à la dissertation, G. Lanson affirme nettement que les exercices de rhétorique ne sont plus adaptés à un public qui n'a pas pour vocation de faire des métiers qui relèvent de la plume: "Notre enseignement classique [...] est plus mauvais que bon, pour tous ceux qui ne sont pas destinés à être vaudevillistes, romanciers, poètes, critiques ou journalistes [...] et c'est le grand nombre tout de même qui est dans ce cas ».

Un demi-siècle plus tard, ceux que l'on appelle les «modernes» rejettent la même dissertation et l'histoire littéraire sous prétexte que «cet usage de la littérature est, sauf pour quelques esprits, parfaitement insipide" (Cahiers pédagogiques, 1954). Le 
mouvement de contestation de la dissertation (l'essai littéraire) se poursuit dans les années 70 jusqu'à promouvoir, toujours au nom d'une meilleure adaptation au public scolarisé, de nouveaux exercices tels le résumé, l'argumentation, la lecture méthodique en lien, pour cette dernière, avec les théories immanentes des textes (linguistique structurale puis textuelle, sémiotique des textes, nouvelle critique). Comme l'attestent les premiers numéros de Pratiques, il s'est agi, sur des bases militantes d'une revendication de scientificité conjointe à une volonté d'innovation pédagogique, de tenter de répondre à un nouveau public scolaire, à la suite des bouleversements démographiques (si l'on en croit la Direction de l'évaluation et de la perspective du ministère de l'Éducation nationale - DEP, il n'y avait que $4,9 \%$ d'une classe d'âge à atteindre le niveau bac en 1950, le nombre passera à 9,8\% dans les années $60,19 \%$ dans les années 70, et atteindra le pic de 62,7 \% en 1995, avant de fléchir légèrement par la suite). C'est ce qui explique qu'en 1974 , on pouvait écrire dans l'éditorial du n $1 / 2 \mathrm{de}$ Pratiques (p. 4) : «On peut cependant hasarder sans grand risque que l'enseignement du français destiné traditionnellement à une élite socioculturellement reconnaissable et reconnue, rencontrait un écho auquel il ne peut plus répondre dans ses nouvelles conditions d'application. »

En remontant vers le présent, comme le rappelle M. Baconnet (2010), les données sont encore plus spectaculaires: en 1960, 1453000 élèves en collège public et privé et 3164000 en 1999; de même au lycée d'enseignement général et technique, 421000 élèves, public et privé, en 1960 et 1464500 en 1999, à quoi s'ajoutent 696000 en lycée professionnel. C'est donc le même argument de l'adaptation au public qu'utilise A. Viala (2001 : 53) pour justifier la pertinence des nouveaux programmes des lycées :

«Le changement majeur au lycée, dans les vingt dernières années, tient à la démocratisation: doublement du nombre d'élèves dans les années 80 , puis doublement de ce doublement, à nouveau, dans les années 90 . Ce fait essentiel suscite une question sociale et politique : faut-il donner la même culture à tous les lycéens, ou opter pour des différences selon les filières. [...] La démocratisation suppose une culture commune : sans quoi, elle n'est que massification ».

À propos du public scolarisé d'aujourd'hui, au lycée ou à l'Université, on est en possession d'enquêtes (De Singly, 1989 ; Baudelot \& Establet, 1992 ; Bautier \& Rochex, 1998 ; Baudelot, Cartier \& Detrez, 1999 ; Lahire, 1997 ; Dubet, 1991; Donnat, 2009...). Elles montrent que les pratiques scolaires de la lecture et de l'écriture sont fort éloignées de leurs usages sociaux ordinaires et que l'intérêt pour la lecture des textes littéraires décroit pour les garçons en premier lieu et pour les filles ensuite quand ils passent du collège au lycée - voir l'enquête (qui mériterait d'être renouvelée) de C. Baudelot et M. Cartier (1998) sur les lectures des collégiens et des lycéens. Il ressort aussi de ces travaux que la lecture littéraire - entendue au sens le plus large - est concurrencée par la multiplication des moyens de communication de masse (télévision, cinéma, internet, etc.), y compris pour les cadres, dont les enseignants. Il est incontestable, là encore des enquêtes l'attestent, que les lycéens, comme les étudiants, de plus en plus, préfèrent l'internet aux sources livresques, ne constituent guère de bibliothèque personnelle et revendent quasi systématiquement les ouvrages qui étaient au programme de l'année écoulée. Un récent numéro du Débat (2012) réactive la controverse entre les opposants à l'internet qui lui reprochent d'accorder une prime à l'opinion au détriment de la réflexion et ses partisans qui lui reconnaissent la possibilité de multiplier l'accès aux savoirs grâce à ces machines à lire et à écrire que 
sont l'Ipad, l'Iphone, l'E-book, le net-book sans parler de Google dont on sait l'usage qu'en font collégiens et lycéens.

Face à ces changements, on peut s'alarmer, comme E. Vigne (2008) ou R. Simone (2012) du fait que l'internet a suscité de nouvelles manières de lire et d'écrire qui s'inscrivent plus dans le fragmenté, le copié-collé, le zapping textuel, que dans la continuité d'une œuvre. Inversement, D. Maingueneau (2013) typologise les genres du numérique et M.A. Paveau (2013, à paraitre) rend compte de «l'inventivité technnosémiotique » de l'internet. De même, C. Becchetti-Bizot (2012) témoigne de l'apport possible des nouvelles technologies à l'enseignement de la littérature. À l'encontre de ceux qui font de la cyber-écriture (des SMS, des mails...) et d'un usage intensif de plateformes comme Facebook la cause de la baisse du niveau en orthographe ou en lecture, des études montrent que ce sont des espaces de création verbale intéressants (voir Ahr et al., 2012). Il apparait que les scripteurs ordinaires éprouvent la jouissance poétique des transgressions des rapports phonie/graphie ou que, au niveau des scripteurs-experts, les fictions (voir les nombreux romans par mail pour adolescents) exploitent les attendus du médium pour créer un nouveau genre romanesque. À quoi s'ajoutent les liens qui peuvent être établis entre les pratiques d'écriture inhérentes aux nouvelles sociabilités juvéniles (Facebook) et les genres du fragment ou du biographique - voir les sites de "fanfiction » décryptés par H. Sagnet (2009). Pour une réflexion plus générale sur l'intérêt et les craintes que suscite la culture du numérique au niveau des usages sociaux, de l'école et de la recherche, je renvoie à M. Wieviorka (2013).

Dans ce contexte de mutations en cours, s'ajoutent la perte de prestige des formations en lettres (voir l'analyse d'A. Boissinot, 2001) face aux formations techniques et scientifiques et le déclin de la littérature dans le "discours social» comme le diagnostique lucidement A. Compagnon (2000). Même si, ponctuellement, sous la forme de livres (Etchegoyen, 1990) ou d'articles (voir David Brunat, «Les littéraires ont toute leur place dans l'entreprise », Le Monde, 30/04/13), la presse et les médias nous assurent que les entreprises s'intéressent de plus en plus aux étudiants de lettres, la réalité des débouchés est tout autre.

Le seul moyen de mesurer le degré de validité des discours de déploration à propos de la baisse généralisée du niveau des élèves (Jarrety, 2000 ; Joste, 2002 ; etc.) est de mener des enquêtes ou de s'appuyer sur des travaux de type historique. Pour les premières, dont on peut regretter qu'elles ne soient pas assez nombreuses et actualisées, on aura recours aux bilans nationaux sur les Pratiques culturelles des Français, aux travaux de l'observatoire national de la lecture ou aux observations plus modestes à hauteur de plusieurs établissements ou d'une cohorte d'élèves. On se référera, pour le primaire, à P. Ceysson et F. Quet (2006); pour le collège à C. Baudelot et M. Cartier (1998), D. Manesse et I. Grellet (1994), à la synthèse de plusieurs enquêtes effectuées par S. Ahr (2005) ou à M. Rémond (2007) ; pour le lycée, à M.-P. Schmitt (2006). Dans tous les cas, ces études procurent une meilleure connaissance de la réalité sociale des lectures des Français et des rapports entre lectures scolaires et usages non scolaires des livres (Demougin \& Massol, 1999). Elles nous permettent aussi de mieux appréhender les acteurs présents dans les situations pédagogiques (canons et corpus de textes scolarisés, types de lectures pratiquées en classe, goûts et représentations des enseignants (en exercice ou en formation) et des élèves en matière de textes littéraires (Legros, M. Montballin \& Vander Brempt, 1991; Monballin \& Legros, 1994 pour la Belgique ; Massol, 2001 ; De Beaudrap, 2004 pour la France). 
Quant aux travaux historiques, on se souvient des regrets formulés par G. Genette $(1969$ : 23) il y a déjà un demi-siècle :

« À vrai dire, notre culture s'intéresse médiocrement à l'histoire des méthodes et des contenus de l'enseignement. Il suffit de considérer la façon naïve dont l'opinion se passionne autour de chaque projet de réforme pour constater qu'il s'agit toujours, dans la conscience publique, de la réforme de l'enseignement, comme s'il s'agissait de "réformer" une fois pour toutes un enseignement vieux comme le monde mais entaché de quelques "défauts" qu'il suffirait de corriger pour lui donner la perfection intemporelle et définitive qui lui revient de droit : comme s'il n'était pas de la nature et de la norme de l'enseignement d'être en réforme permanente. [...] Or il est bien évident, au contraire, que l'enseignement est une réalité historique qui n'a jamais été ni transparente ni passive: les structures de savoir et celles de l'enseignement ne coïncident jamais parfaitement [...]. D'autre part, les méthodes et les contenus de l'enseignement participent - éminemment de ce que Lucien Febvre appelait l'outillage mental d'une époque, et par là encore ils sont l'objet d'histoire. »

Heureusement, dans l'intervalle, même si les futurs enseignants de lettres sont tenus dans l'ignorance de leurs résultats, et de ce fait, incapables de surmonter leur crise d'« identité professionnelle » (Bertucci, 2004), les recherches à propos de l'histoire de la discipline se sont multipliées, comme en témoigne A. Chervel (2006), qui écrit : «Faire l'histoire d'une discipline scolaire, c'est s'attacher non seulement aux programmes et aux finalités de la discipline, non seulement aux pratiques éducatives et enseignantes qu'elle met en œuvre mais également aux effets réels et concrets qu'elle produit sur les élèves et la société qui les entoure ».

Quant à la "crise interne" de la discipline, elle se manifeste par le fait que l'enseignement du français au niveau de l'enseignement du secondaire est aujourd'hui une discipline dont la "matrice disciplinaire » a perdu de sa cohérence à la suite, comme on va le voir, des transpositions didactiques opérées à partir de nouvelles théories, issues en particulier de la linguistique. Elles vont progressivement gagner leur part de reconnaissance institutionnelle après 1968 et renouveler la «lecture littéraire ».

\section{La « lecture littéraire » : aspects théoriques et didactiques}

54 Je dirai qu'afin de mesurer la part des "continuités et des ruptures dans l'enseignement de la littérature ", pour reprendre le titre du numéro 168 du Français aujourd'hui (2010), j'ai centré l'étude qui suit sur «la lecture littéraire» en situation scolaire telle qu'elle a évolué en 40 ans. Il apparaitra, au fil de la recherche, qu'avec cette activité et les tâches qui lui sont subséquentes, l'on est en présence d'une modélisation, plus empirique que théorique et soumise à la variation, qui concerne tant les objets donnés à lire (les corpus), l'appellation de l'activité et les modalités de sa mise en œuvre (" analyse de textes », « lecture de textes », « lecture méthodique », « lecture analytique", "lecture plurielle», "lecture littéraire»). Je ne chercherai pas fondamentalement à proposer une «modélisation de la lecture littéraire » (Tauveron, 2009), ni une nouvelle conceptualisation de cette pratique dont J.-L. Dufays $(2005,2006)$ a bien montré le « flou conceptuel » de la notion et le fait qu'elle est l'objet de conflits définitionnels (d'où mon usage des guillemets quand j'y fais référence). Je vais plutôt 
tenter de rendre compte, en les problématisant, des usages scolaires d'enseignement/ apprentissage de la lecture de textes reconnus comme littéraires, car, comme l'écrit B. Daunay $(2007: 168)$ :

« Dire de la notion de lecture littéraire qu'elle est un outil didactique, c'est signaler son intérêt et sa productivité dans les recherches didactiques concernant l'enseignement de la littérature. Pour autant ce n'est pas là un concept construit de manière consensuelle dans le champ : nul ne saurait définir, sans se voir opposer une autre définition, ce qu'est une lecture littéraire. Il faut insister ici sur son statut de notion heuristique, en ce qu'elle permet d'interroger l'acte de lecture (scolaire notamment) et de concevoir un enseignable qui ne soit pas seulement le texte (et ses divers contours), mais la relation texte-lecteur.

Enseignable qui a évolué dans ses contenus comme dans ses formes, depuis les années 70-80, comme je vais le montrer maintenant. Pour ce faire, dans le prolongement de la revue de questions réalisée par J.-L. Dufays et alii (1996a, 1996b), j'effectuerai une brève synthèse des théories de référence qui ont été transposées dans Pratiques à propos de l'enseignement de la littérature et de la lecture littéraire.

\subsection{L'immanentisme textuel et ses applications pédagogiques}

La décennie 60-70 correspond à un mouvement de rénovation théorique que l'on doit à des francs-tireurs universitaires et dont témoigne la création de revues dont Littérature, qui présente Pratiques comme une "revue sœur» ou l'organisation de colloques consacrés à la littérature et à son enseignement (Doubrovsky \& Todorov, 1971; Mansuy, 1977). Au cours de ces années, s'opère en particulier une réactivation des formalistes russes des années 20 ou du cercle praguois (voir la présentation de T. Todorov, 1965) à qui l'on doit les notions de " défamiliarisation » ou "d'étrangeté " et de « désautomatisation » du langage qui rendraient compte avec la « réflexivité » des procédés de littérarisation. C'est à cette époque, au nom du critère de la « littérarité » et de l'« autotélie » de l'œuvre littéraire, que la stylistique littéraire va entrer en crise. Il est intéressant, à cet égard, de constater - je me limite aux seuls travaux linguistiques ou apparentés - l'émergence de ce que G. Antoine (1966) appelle la "stylistique des formes", en référence à la sémantique structurale, la sémiotique narrative et la poétique. Pour différents que soient ces paradigmes, ils partagent des postulats qu'explicite P. Guiraud (1963) : le texte littéraire est un objet linguistique, qui doit faire l'objet d'une étude immanente. Il s'en suit que la notion de "littérarité ", assimilable à la notion de "diction" au sens de G. Genette, prendra des formulations diverses : «fonction poétique» (Jakobson, 1963) ; «langage de connotation» (Barthes, 1970); «clôture de l'énoncé » (Arrivé, 1972) ; "texte poly-isotopique » (Arrivé, 1973, 1975); "potentialité de polyfonctionnalité » (S.-J.Schmidt, 1973); «disponibilité poly/ diasémique " (Peytard, 1982). Ce textualisme émergent est en phase avec la stylistique anglo-saxonne de l'époque et du New Criticism anglo-américain (Wellek \& Warren). « La stylistique est une branche de la linguistique, mais une branche concernée par le traitement des variables dans un texte dans son entier » affirme R. Fowler (1966). On va alors assister à ce que M. Marghescou $(1974,2009)$ appelle la «textophilie euphorique généralisée " qui sera le fait des linguistes (R. Jakobson, A.-J. Greimas, N. Ruwet, H. Meschonnic, J. Peytard...), des poéticiens (J. Kristeva, G. Genette, M. Riffaterre, U. Eco...) ou à des représentants de l'avant-garde littéraire (Tel Quel, Promesse...). En témoignent, outre les livres de ces auteurs, la création de revues telles Langue française, Langages ou Poétique ou le numéro de La Nouvelle Critique (1969) consacré aux actes du 
colloque de Cluny (16-17 avril 1968) intitulé «Linguistique et Littérature». Cette hégémonie grandissante de la linguistique structurale parmi les sciences humaines sera bientôt suivie par les travaux relevant de la narratologie (Propp, Greimas, Brémond, Genette, Hamon, Mitterand etc.). Au niveau de l'enseignement, les articles qui paraitront dans les revues telles Bref, Le Français aujourd'hui, Pratiques ou Les Cahiers du Crelef s'en feront l'écho. C'est ainsi que les premiers numéros de Pratiques donneront la parole à des théoriciens (R. Barthes, 1975; A.-J.Greimas, 1976) ou à des écrivains (J. Thibaudeau, 1974 ; C. Ollier, 1975 ; J. Ricardou, 1975). C'est dans ce contexte et sur ces bases théoriques que ces revues initieront les recherches concernant ce que l'on appelait alors la «pédagogie du français ». À titre d'exemple, dans le numéro 1/2 de Pratiques, sous la plume de J.-F. Halté, R. Michel et A. Petitjean (1974), on trouve une analyse dite «structurale » d'un conte de Poe (Le Chat noir) : chacun pourra constater qu'il s'agit avec cette lecture interprétative du conte, d'un bel exemple d'applicationnisme des théories structurales mâtinées de psychanalyse un peu rudimentaire. Au cours des années qui suivront les numéros de Pratiques vont multiplier les objets d'études (catégories narratives, type et genres de textes, cohésion textuelle, relations textuelles, modes et régimes énonciatifs...), accordant une place privilégiée à la fiction narrative dans le corpus des textes étudiés, tandis que prédomineront les aspects formels dans les activités de lecture et d'analyse des textes.

Au même moment se développent aussi les travaux qui relèvent du FLE (voir des revues comme les ÉLA ou Le Français dans le monde). Pour ce qui concerne plus spécifiquement la didactique de la littérature en FLE, on peut dire que les textes littéraires qui formaient le socle de base de l'enseignement patrimonial dit «traditionnel » où l'on enseigne la langue à partir de la littérature, ont été, dans un premier temps, tendanciellement exclus du domaine de l'apprentissage de la langue. Le rejet a été sans appel quand, dans les années 60 le courant SGAV (stucturo-global audio-visuel) et les méthodes audio-orales et audio-visuelles, se sont imposés, privilégiant la compétence orale et le français fondamental (voir Rivenc, 1964 et Cortier \& Parpette, 2006). Sans être exclus, les textes littéraires ont été ensuite fortement concurrencés, dans les années 80 , par des documents dits "authentiques» pluri-codés de nature diverse (journaux, publicités, images, etc.). Selon l'approche communicative et sur la base d'une conception anthropologique de la culture, ils étaient censés être mieux adaptés aux nécessités fonctionnelles et communicatives des échanges langagiers (voir Moirand, 1982; Porcher \& Zarate, 2001). Au mieux, les œuvres littéraires étaient ravalées au rang de "document», comme les appelle J.-C. Gagnon (1985) et cohabitaient avec des textes fabriqués - dialogues, sketches -, voire avec des textes littéraires réécrits sous une forme dite « simplifiée » (voir Le Candide " facile »).

Dans un second temps, comme le rappelle A.Séoud (1997), le texte littéraire a progressivement été réhabilité sous la forme d'approches formelles des textes (Peytard, 1982, 1988) et a été utilisé comme un support réservé aux classes de niveau dit " avancé » ou "niveau 3 " permettant de tester les apprentissages antérieurs qui habilitent les apprenants à lire ces textes. Comme l'écrit D. Coste en 1982, « le passage à l'appréciation littéraire n'est concevable qu'une fois établies les fondations de la langue parlée et de la langue écrite usuelle ». La littérature retrouvera ensuite toute sa place dans l'enseignement du FLE pour ses vertus "anthropologique» (Porcher, 1988) et « représentative ». C'est ainsi que J. Mounin (1976) affirme qu'elle accomplit sa fonction 
« documentaire » à propos de «la culture d'un pays donné » bien mieux que ne le fait un « document authentique » qui a l'inconvénient d'être très vite périmé.

L'intérêt affiché et revendiqué de l'apport structural immanentiste était double, à la fois critique et propositionnel.

Critique, voire polémique, au sens où les approches poétiques et linguistiques des textes ont rendu problématiques certains présupposés théoriques de la version scolaire de l'histoire littéraire : l'évidence des intentions de l'auteur, la monosémie des textes et leur transparence référentielle, l'instrumentalisation psychologique et moralisatrice des œuvres. C'est ainsi que dans le même numéro 1/2 de Pratiques (mars 1974), dans le prolongement des articles de R. Barthes (1971), J.-L. Houdebine (1971), J. Thibaudeau (1971), F. Rastier (1972) ou de P. Kuentz (1972), on trouve, signé par J.-F. Halté et A. Petitjean, un article critique d'un manuel («Le Lagarde et Michard»), représentatif de ce qu'il était alors convenu d'appeler la pédagogie traditionnelle. On lui reproche sa conception de la périodisation littéraire par siècle et une imagerie littéraire héritée du positivisme et de l'historicisme du XIX ${ }^{\mathrm{e}}$ siècle, un panthéon d'auteurs et des extraits de chefs-d'œuvre valorisés comme des modèles moraux, intellectuels et esthétiques (sélection de "belles pages »), l'aplatissement des œuvres sur la biographie (la «viœuvre »comme l'appelle A.Compagnon, 1998). À la mise en cause du manuel s'ajoute une critique non moins radicale des exercices officiels tels que la « dissertation » et l'« explication de texte» (Delesalle, 1970). Pratiques reviendra par la suite sur ces exercices canoniques liés à la pratique du commentaire, qui vont certes évoluer, mais demeurent discutables sur le fond (voir Charolles, 1990, et l'ensemble du numéro 68 de Pratiques; Daunay, 1997 ; Delcambre, 1989, 1990 ; Denizot, 2013). Ces critiques des différents aspects de la «forme scolaire» (Vincent, 1994 ; Reuter et al., 2007) de l'enseignement de la littérature sont d'autant plus justifiées dans le contexte des années 70 que l'on assiste à un début de rapprochement des deux ordres (primaire et secondaire) d'enseignement (de la réforme Capelle-Fouchet de 1963 à l'instauration en $1975 \mathrm{du}$ " collège unique »). Ce qui signifie que cet enseignement de la littérature, destiné à "une élite de jeunes bourgeois cultivés", comme l'écrit S. Delesalle, ne saurait être adapté au nouveau public qui va progressivement, des CEG (collèges d'enseignement généraux) aux futurs CES (collèges d'enseignement secondaire), accéder à ce niveau d'études et qu'il faudra repenser en profondeur la discipline. Ce que propose en 1969 le Manifeste de Charbonnières, texte fondateur de l'Association française des enseignants de français (AFPF), aux origines de l'AFEF dont le Français aujourd'hui va devenir la revue de référence. Le collectif de Pratiques, dès sa création s'associera à ce mouvement de rénovation qui se heurtera aux résistances de l'Inspection générale et de l'Université détentrice de la tenue des concours de recrutement des professeurs du second degré. C'est ainsi que dans le numéro 3/4 de Pratiques, nous publierons le texte consacré à « la lecture des textes " émanant de la commission Pierre Emmanuel et censuré par le Ministère. Se trouvent ainsi confirmés les travaux sociologiques qui dénoncent la fonction conservatrice de l'École et son fonctionnement dans la reproduction des inégalités sociales ainsi que le rôle spécifique de l'enseignement de la littérature en la matière (Baudelot \& Establet, 1971 ; Bourdieu, 1971b ; Bourdieu \& Passeron, 1964).

61 Propositionnels, les premiers travaux de didactique et les théories auxquelles ils réfèrent l'ont été dans la mesure où ils ont servi d'antidote à l'impressionnisme (y compris sous sa forme phénoménologique (G. Poulet, J.-P. Richard) ou psychanalytique 
(M. Bonaparte, C. Mauron) censée permettre d'accéder à la " pensée indéterminée » ou à la « conscience profonde » qu'expriment les œuvres. Pour ce faire, l'accent sera mis sur les indices formels susceptibles d'étayer les interprétations et qui ont l'avantage de fournir à la discipline des savoirs objectivables et des exercices évaluables, procurant, de ce fait, un regain de légitimité aux études de lettres par rapport aux disciplines scientifiques. À ce propos, en rupture avec le psychologisme néo-beuvien et par rapport au modèle lansonien dégradé de l'analyse scolaire des textes, on postule alors, comme le dénoncera plus tard P. Ricoeur (1986: 183) que «le texte serait une machine au fonctionnement purement interne auquel il ne faudrait poser aucune question réputée psychologisante -, ni en amont du côté de l'intention de l'auteur, ni en aval du côté de la réception par un auditoire, ni même dans l'épaisseur du texte du côté d'un sens, ou d'un message distinct de la forme même ».

La distance avec la méthodologie historique se traduit aussi par l'affirmation qu'il existerait un statut particulier des textes "littéraires", à savoir que la fonction référentielle s'y trouve abolie au profit de potentialités symboliques et qu'ils seraient, de ce fait, nécessairement polysémiques, comme l'affirme métaphoriquement R. Barthes (1984) : «Si, jusqu'à présent, on a vu le texte sous les espèces d'un fruit à noyau (un abricot par exemple, la pulpe étant la forme, l'amande étant le fond), il convient de le voir maintenant plutôt sous les espèces d'un oignon, agencement superposé de pelures, dont le volume ne comporte finalement aucun cœur, aucun noyau, aucun secret, aucun principe irréductible, sinon l'infini même de ses enveloppes. »

En fonction de quoi M. Naturel (1995) peut affirmer : «Il apparait donc clairement que le texte non littéraire a un sens et un seul et que le texte littéraire permet une lecture plurielle; d'une part, il peut être abordé sous différents angles d'analyse et d'autre part, il se prête à de multiples lectures et donc à de multiples interprétations ».

64 A. Séoud (1997: 15), dans son ouvrage consacré au FLE ne dit pas autre chose: "L'écrivain écrit pour l'éternité et Henri Besse fait remarquer pertinemment qu'on ne lit pas un journal vieux d'un an mais on prend du plaisir à lire tel écrivain de l'Antiquité. Cette qualité, le texte littéraire la doit à sa polysémie, à sa richesse inépuisable de sens, qui fait que, par delà même parfois des frontières de la langue, il peut parler à tout le monde ».

Cette conception de l'œuvre littéraire dont on sait la filiation avec la réflexion esthétique du romantisme allemand (voir Lacoue-Labarthe \& Nancy, 1978) fera l'objet d'un examen critique (Arrivé, 1975 ; Aron, 1984a ; etc.) et sera d'autant mieux invalidée qu'elle ne saurait rendre compte d'une spécificité des textes littéraires. Il est assez étonnant, de ce point de vue, de voir M. Thérien (1997) tenter malgré tout une définition du «littéraire» sur la base de quatre critères (polysémie, régularité de forme, intertextualité, ouverture sur l'imaginaire) qui ont l'inconvénient de convenir aussi pour certains messages publicitaires. Ce qui n'est guère satisfaisant, il faut bien le reconnaitre. Je ne suis pas plus persuadé que les quatre critères qu'avance V.Jouve (2010), à savoir que «le sens littéraire [...] est divers, il n'est pas entièrement conceptualisé, il éclaire des dimensions de l'humain, il passe en grande partie par la forme " soient plus distinctifs. Certains de ces traits sont des attributs des "discours constituants ", d'autres, un marqueur plus spécifique des fictions relevant de la sphère de la "production restreinte» au sens bourdieusien du terme. En fait, comme le propose Y. Reuter (1990: 10), à la suite de M. Charles (1985), la littérarité n'est ni le texte, ni le commentaire, mais « le rapport texte-commentaire, lui-même intégré dans 
un champ spécifique et des institutions qui lui sont liées ». Ce point de vue est partagé par M. Marghescou (1974) qui écrit :

«Face à une certaine catégorie de textes (les textes littéraires), le lecteur suppose immédiatement la présence d'un sens caché, même si le sens apparent est cohérent et clair. On se sent comme obligé, en lisant ces textes, de résoudre une énigme, de découvrir un symbole, de reconnaitre un archétype. Une longue préparation, qu'une ontologie culturelle fera remonter aux lectures en classe et une phylogénie culturelle, à l'interprétation des textes sacrés du Moyen Âge, en est responsable. »

\subsection{Les théories sociologiques pour une socio-didactique}

La sociologie est le second paradigme que Pratiques va promouvoir et qui a alimenté de nouvelles recherches en didactique de la littérature.

Il s'agit, en premier lieu de la sociologie de la littérature (Bourdieu, 1971a, 1992; Dubois, 1978, 1980 ; Charle, 1978 ; Viala, 1989) dont les travaux portent sur la « question sociale de la littérature " (Reuter, 2000). On se souvient, à cet égard, que R. Barthes (1963-1993) affirmait que "l'histoire littéraire n'est possible que si elle se fait sociologique ", c'est-à-dire que si elle prend en compte les modalités de sa "production-consommation-diffusion » de la littérature, car ces dernières relèvent de la définition même de la littérature. De ce point de vue, on doit à P. Bourdieu la notion de « champ » sous la forme d'un système d'agents (éditeurs, auteurs, critiques, lecteurs) et de positions qui les unissent ou les opposent. Ce champ est lui-même conçu comme une structure bi-polaire qui, sur la base de la production de ces biens à la fois matériels et symboliques que sont les œuvres, oppose la sphère de la production "élargie " (dominée par la rentabilité marchande) à celle de la production "restreinte » (valorisant la reconnaissance symbolique). Selon qu'ils appartiennent à un champ ou à l'autre, les écrivains privilégient la qualité littéraire de leur œuvre et leurs aspirations artistiques aux dépens de la rentabilité financière, à l'inverse de ceux qui recherchent les gros tirages en concordance avec les goûts et les attentes d'un large public. Comme je l'ai montré (Petitjean, 2011) un auteur dramatique tel B.-M. Koltès a parfaitement conscience de cette "double façon d'être écrivain", lui qui déclare - on croirait entendre Flaubert - détester "la médiocrité en art», ne pas vouloir "écrire pour les épiciers", prendre du plaisir « à aller à contre-courant », tout en soulignant le manque à gagner financier de ce choix ( Il faudrait que j'écrive des pièces policières pour la radio pour faire des sous... »). C'est là, comme l'a montré N. Heinich (2000), l'une des figures possibles de l'écrivain (le régime "inspiré») ou d'une des manières (le "joueur mordu ») de participer à ce que B. Lahire (2006) appelle le « jeu littéraire ».

Quant à la théorisation de l'institution proposée par J. Dubois et illustrée dès les années 80 , entre autres publications par les numéros 42 et 44 de Littérature, elle permet de comprendre comment l'institution littéraire fait système avec d'autres institutions (édition, presse, école...) pour participer à l'émergence, à la reconnaissance et à la consécration des auteurs. Comme le résume J.-M. Klinkenberg (1998), appréhender la littérature comme une institution

« l'arrache à la pureté idéale que s'attribue cette dernière et brise avec le mythe qui la présente comme une activité gratuite et désincarnée [...] ce mythe est lui-même une construction de l'institution littéraire du xIx siècle [...] en élaborant l'image du Poète, image qui rétablit la suprématie d'un individu sur les autres en faisant de sa pratique la marque d'une nature d'élite. Ce mythe est d'ailleurs toujours prégnant de nos jours: la littérature reste pour beaucoup une haute solitude où l'écrivain, 
mage inspiré que la société a le devoir d'écouter, n'a de compte à rendre qu'à sa

conscience et à son inspiration, laquelle débouche sur l'œuvre ». particulier scolarisés dans l'enseignement professionnel (Ledur, 1996), ont une pratique peu autonome et intensive de la lecture de textes littéraires. Les causes de leur résistance à l'acculturation littéraire sont bien connues : préférences pour les textes relevant essentiellement de la sphère «élargie » de la production (en proximité avec l'univers référentiel ou convoquant l'irrationnel) ; valorisation de la lecture psychoaffective ; difficultés à utiliser de manière cohérente les données d'un texte, insécurité interprétative face aux indéterminations des textes valorisés scolairement comme littéraires et rejet subséquent de ces derniers. Le désintérêt pour la lecture des textes littéraires va s'accroissant plus les élèves avancent dans le cursus et il est nécessaire, même s'il existe d'autres facteurs, de s'interroger sur les effets de l'enseignement en la matière.

lecteurs. C'est ainsi que G. Mauger, C. Poliak et B. Budal (1999), à l'aide de l'analyse d'entretiens, explicitent les usages sociaux de la lecture (types de bibliothèque, fonctions de la lecture, manières de lire...).

Concernant les retombées des recherches de type sociologique, on se référera aux différents articles de Pratiques qui historicisent les notions de littérature, de littérarité, d'institution littéraire, que ce soit par l'intermédiaire des notions de champ littéraire et d'instances de légitimation, d'histoire littéraire (périodisation, courants, mouvements, écoles, groupes, biographie construite, etc.). À titre d'exemples: J. Dubois (1980) ; Y. Reuter (1981) ; D. Dupont et J.-M. Rosier (1983) sur le fonctionnement de l'institution littéraire ; J.-P. Goldenstein (1983b) sur les instances qui reconnaissent et consacrent les écrivains; J.-P. Bertrand et alii (1983) sur le surréalisme et A.Pages (2000) sur le naturalisme. Voir aussi les numéros 32 («La littérature et ses institutions ») et 38 («Enseigner la littérature») de Pratiques ainsi que les numéros 50 («Les paralittératures») et 54 ("Les mauvais genres»), dans lesquels on cherche à donner 
aux élèves les moyens de réfléchir au statut (corpus, fonctions) de la littérature. On retiendra aussi les numéros ou les articles qui présentent l'intérêt, à des fins didactiques, d'interroger les représentations sociales de la figure de l'écrivain (Pratiques, $\mathrm{n}^{\circ} 27$ ) et de problématiser les notions de biographie (Pratiques, $\mathrm{n}^{\circ} 45$ ), de genre (Pratiques, $\mathrm{n}^{\circ} 62$; K. Canvat, 1992; N. Denizot, 2010) et de style (Pratiques, $\mathrm{n}^{\circ}$ 135-136).

Pour l'une des transpositions didactiques les plus abouties en matière de sociologie de la littérature et de "travail d'institution des lecteurs» (Privat, 1993), on se reportera aux deux tomes du Manuel d'histoire littéraire de D. Dupont, Y. Reuter et J.-M. Rosier (1988), dont l'objectif est d'effectuer des démarches de familiarisation culturelle du type :

- initiation aux règles institutionnelles qui régissent la structuration du champ littéraire: typologie des agents qui le constituent (écrivains, critiques, éditeurs, libraires etc.) ; modes d'organisation (cénacles, écoles, mouvements, etc.) ; instances qui participent à l'émergence, à la reconnaissance et à la consécration des auteurs; valeurs en partage (nouveauté versus répétition selon le champ de la production (restreinte ou élargie);

- problématisation du métalangage universitaire sur la littérature et des notions que l'on y utilise (littérature ; auteur, biographie, genre, style, valeur...);

- travail sur l'écrivain, son statut (écrivain vocationnel et inspiré ou grand public), ses fonctions et ses modes d'écriture selon son champ éditorial d'appartenance et les postures qu'il adopte (éveilleur de conscience, travailleur du verbe, amuseur public...) ;

- étude des péritextes (lettres, brouillons, ébauches...) comme sources d'information pour l'interprétation des textes;

- études des paratextes (préfaces, manifestes...) qui explicitent les codes génériques ou les transgressions stylistiques.

76 C'est ainsi que J.-M. Rosier (1998), désireux de prendre en compte les représentations des lycéens belges concernant l'institution littéraire et la littérature francophone de Belgique, propose des questionnaires portant sur des rubriques du type :

«a) Nos pratiques culturelles (questions du type : Qui lit quoi ? Quand ? Où ?)

b) Nos acquis scolaires (questions et définitions visant à appréhender l'appareil conceptuel scolaire déjà intériorisé: Qu'est-ce qu'un auteur? Qu'appelle-t-on littérature ? Qu'est-ce que le Romantisme ?...)

c) Notre système de valeurs en matière de hiérarchie culturelle (La littérature estelle plus importante que le cinéma ? La B.D. est-elle un genre mineur ?...)

d) Notre savoir pour tout ce qui concerne le fonctionnement du champ culturel (Combien y a-t-il d'écrivains dans notre pays ? Citez des maisons d'édition (Qu'estce qu'un prix littéraire ?) et celui du milieu culturel ambiant (La ville où se situe l'école possède-t-elle des cinémas, un théâtre, des librairies...) ».

Dans le deuxième tome du Manuel d'histoire littéraire, est ajoutée au point de vue sociologique une dimension historique qui permet de rendre compte de l'évolution du champ littéraire au cours des siècles.

Ce qui permet de ne pas se limiter à une version faible de l'ouverture du texte sur son contexte (mention d'une source ou d'une clé référentielle), mais d'initier les élèves aux règles institutionnelles qui régissent la production des œuvres. La démarche a du sens en FLM mais est aussi intéressante en FLE car elle permet à l'étudiant d'observer le fonctionnement du champ littéraire de la France et de le comparer à celui, s'il existe, de son pays d'origine. À ce propos, J.-M. Rosier (1998) suggère des questionnaires soumis aux lycéens belges, qui sont autant d'entrées en histoire de la littérature : 
"-Comment expliquer le succès des symbolistes belges à Paris?

- Quelle stratégie de légitimation est à l'œuvre dans la biographie d'Hergé ou de Simenon?

- Pourquoi Plisnier reçoit-il le Goncourt pour Faux Passeports?

- Pourquoi le théâtre-action s'est-il développé en Belgique francophone?

- Quelles raisons peuvent expliquer l'existence d'un groupe surréaliste à la

Louvières?

Ces questions (situations-problèmes) sont des titres de séquences pédagogiques, lesquelles portent sur des points d'histoire de la littérature. Ces exercices touchent aux aspects d'une lecture informée par les paramètres les plus divers: textuels, littéraires, historiques, culturels ».

\section{lycées aient supprimé la rubrique "Écrire, publier, lire » du texte officiel de 2000} renforçant de ce fait l'attitude de sacralisation du littéraire.

\subsection{Lire et interpréter les textes}

Le troisième paradigme à l'origine d'un autre regard didactique sur la lecture littéraire va consister à mettre l'accent sur l'activité du sujet lecteur. Parmi les différents travaux qui relèvent de cette problématique, on peut distinguer les positions de R. Barthes (1970) et ses différents « codes de lecture "; M. Picard (1986) et sa partition entre liseur, lu et lectant ; V. Jouve $(1992,1993,2011)$ et sa répartition entre le liseur, le lu et le lisant ainsi que sa théorisation de « l'effet-personnage »; J.-L. Dufays (2010a) et sa théorie des "phases de lecture ». Je mentionnerai aussi la distinction proposée par U. Eco (1985) entre trois types d'intention (auctoris, operis, lectoris) et les réflexions de P. Ricœur (1983) sur la lecture comme "acte de reconfiguration ». À ces différentes conceptions de la lecture, j'ajouterai l'apport plus récent des (im)pertinences théoriques de P. Bayard $(2002,2008)$ et la réflexion non moins décapante d'Y.Citton (2007). Ce troisième paradigme est surtout représenté par les théories de la réception (Jauss, 1978 ; Iser, 1986 ; Jurt, 1983) telle qu'elles se sont illustrées sur des œuvres (Labrosse, 1985) ou des auteurs particuliers (Jurt, 1980). C'est ainsi que I. Vultur (2005) décrit les différents cadres génériques mobilisés par la critique pour catégoriser et assimiler la somme romanesque de M. Proust. On doit aux théories de la réception - que ce soit le lecteur « implicite » d'Iser dont le rôle est « inscrit » dans le répertoire du texte ou le lecteur historique contraint dans sa lecture par son « horizon d'attente » (Jauss) et plus tard à la théorie des "communautés interprétatives » de S. Fish (1980-2007) -, une description de l'acte de lecture. Il est conçu comme une transaction dynamique de significations, un processus dialogique qui met en relation, d'un côté le texte, tel qu'il a été élaboré dans un contexte donné sous l'autorité d'un auteur et dont le sens est à la fois prescrit et indéterminé, et, de l'autre côté, le lecteur. Ce dernier investit le texte de significations différentes selon les enjeux de sa lecture, ses connaissances encyclopédiques, sa compétence linguistique, ses sentiments et ses affects, son système axiologique et plus généralement son identité socioculturelle. Cette conception interactive de la lecture, dans la relation qui unit le texte à un lecteur, met l'accent sur le « pouvoir créatif » du lecteur (Michel, 2011) et sur le fait, comme le souligne Y. Citton (2007), que "toute lecture implique une forte activité projective de la part de l'interprète ». Ce rôle du lecteur comme "maitre du sens ", nombreux sont les auteurs qui ont en conscience de Montaigne à Borges ou Gide, pour ne prendre que trois exemples : 
"Un suffisant lecteur descouvre ès escrits des perfections autres que celles que l'auteur y a mises et aperçueues, et y preste des sens et des visages plus riches » (Montaigne, Essais, T. II).

«La littérature est chose inépuisable, pour la raison suffisante et simple qu'un seul livre l'est. Le livre n'est pas une entité close : c'est une relation, c'est un centre d'innombrables relations. Une littérature diffère d'une autre, postérieure ou antérieure à elle, moins par le texte que par la façon dont elle est lue » (Borges, Enquêtes).

«Avant d'expliquer aux autres mon livre, j'attends que d'autres me l'expliquent. Vouloir l'expliquer d'abord c'est en restreindre aussitôt le sens ; car si nous savions ce que nous voulions dire, nous ne savons pas si nous ne disons que cela. - On dit toujours plus que CELA. - Et ce qui surtout m'y intéresse, c'est ce que j'y ai mis sans le savoir, - cette part d'inconscient, que je voudrais appeler la part de Dieu. - Un livre est toujours une collaboration, et tant plus le livre vaut-il, que la part du scribe y est petite, plus l'accueil de Dieu sera grand. - Attendons de partout la révélation des choses ; du public, la révélation de nos œuvres » (Gide, Paludes).

81 Qu'elle soit individuelle - chacun peut faire l'expérience qu'en lisant une même œuvre à différentes époques de sa vie (enfant, adolescent, adulte), il ne lui accorde pas forcément les mêmes significations - ou collective, elle est soumise. à la variation historique. Écoutons H.-G. Gadamer (1996 : 139) :

«Toute époque comprend nécessairement à sa manière le texte transmis [...]. Le véritable sens d'un texte, tel qu'il s'adresse à l'interprète, ne dépend précisément pas de ces données occasionnelles que représentent l'auteur et son premier public. Du moins, il ne s'y épuise pas. Car la situation historique de l'interprète et, par conséquent, la totalité du cours objectif de l'histoire contribuent sans cesse à le déterminer [...]. Le sens d'un texte ne dépasse pas son auteur, non pas occasionnellement, mais toujours. C'est pourquoi la compréhension est une attitude non pas uniquement reproductive, mais toujours productive. "

À propos de ce déport herméneutique, qu'il soit dû à la distance historique ou au contexte culturel, R. Lamrany (2004) a bien montré qu'un lecteur du xxI siècle et un lecteur du XIX ${ }^{\mathrm{e}}$ siècle ne construisent pas la même représentation mentale d'un poème de Baudelaire, pas plus qu'un lecteur étranger et un lecteur français. On se référera aussi à J. Leenhardt et P. Jósza (1999) qui ont étudié la réception des Choses de G. Perec en Hongrie et en France. Il en va, a fortiori, de même pour le professeur lettré et pour les apprentis lecteurs que sont les élèves. On comprend que se soient développées dans les années 80-90, du côté du FLE, outre la pédagogie communicative et l'approche pragmatique des textes, des recherches prenant en compte la "perspective du lecteur ", pour reprendre le titre d'un numéro spécial du Français dans le monde (1988) ainsi que des travaux sur l'interculturalité (voir Besse, 1988 ; Collès, 1994 ; AbdallahPretceille, 1995 ; Blanchet, 2010, pour un bilan). Ces études montrent que les difficultés de compréhension des textes littéraires, au-delà de la langue, tiennent au fait que l'on ne perçoit l'Autre ou la culture de l'Autre qu'à travers la sienne propre. De même qu'il existe des « cribles linguistiques » qui font que l'on a du mal à entendre les sons d'une langue autre qui n'existent pas dans la sienne ou le système des temps verbaux, par exemple, de même l'enseignement de la littérature se heurte aux phénomènes de «cribles culturels » (Besse, 1984).

$83 \mathrm{Au}$ sein du français langue maternelle (FLM), on assiste progressivement, à la même époque, à une évolution qui va consister à prendre de la distance par rapport à la seule étude interne des textes pour s'intéresser au sujet lecteur. Je renvoie aux numéros de revues de didactique qui témoignent de cette inflexion : Pratiques, nº 76, 1992 ; Repères, 
$\mathrm{n}^{\circ}$ 13, 1996, $\mathrm{n}^{\circ}$ 19, 1999; Le Français aujourd'hui, $\mathrm{n}^{\circ} 137,2002$. Ces changements s'expliquent pour une part, en lien avec la rénovation des collèges et, dans une moindre mesure, avec la réforme des lycées, par l'intérêt croissant pour les démarches d'enseignement et la centration pédagogique sur le sujet «élève », comme l'attestent les thématiques du type pédagogie "par objectifs ", " en projet » (Pratiques, $\mathrm{n}^{\circ} 36$ ), « différenciée » (Pratiques, $\left.n^{\circ} 53\right)$, etc.

Plus précisément, on peut dire que les théories de la lecture, au-delà de leurs incompatibilités paradigmatiques (qu'elles soient d'inspiration sociologique ou herméneutique) et du radicalisme de certains modèles (je songe à la déconstruction et à la dissémination du sens de J. Derrida, 1967, 1972), ont permis de promouvoir une conception interactive de la lecture au sens où elle est une " rencontre entre une offre et une demande » (Goulemot, 1985). Encore faut-il que le professeur mette les élèves dans des situations qui favorisent cette demande à l'aide de situations fonctionnelles de lecture. Ce qui implique de prolonger au collège l'équivalent du coin lecture et de la bibliothèque de classe du primaire et de promouvoir le développement au sein de la classe de sociabilités autour du livre. À titre d'exemple, je citerai l'article de B. Duhamel (1993), qui met en place des dispositifs d'échanges de livres et de lectures, monte des entretiens au cours desquels les élèves prennent conscience de ce qu'implique une lecture autonome. Je renvoie aussi aux activités proposées par M.-C. Vinson et D. Lelièvre-Portalier (1993) sur les médiations culturelles (familiarisation avec les lieux et la culture du livre à l'aide du dispositif qu'elles appellent « la bouquinerie »). Pour le lycée, je fais référence à la création du Goncourt des lycéens (Burgos \& Privat, 1993 ; Burgos, 1994) ainsi qu'aux nombreux concours d'écriture.

Dans le cadre de l'institution (programmes et pratiques pédagogiques), ces travaux, avec le temps, ont pu infléchir les Instructions Officielles depuis 2000 sous la forme de ce que les prescripteurs appellent les «lectures cursives", dont l'objectif est de favoriser le " plaisir » de lire des élèves et de les familiariser avec l'univers des livres, bref de leur permettre d'acquérir une pratique et une culture de l'écrit. Ils les opposent aux «lectures méthodiques" ou "analytiques", mais en faisant le pari, comme le proposent aussi les partisans du « carnet de lecteurs » (Joole, 2009; Ahr \& Joole, 2010) ou des "comités de lecture» (Lebrun, 2004) qu'il est possible de faire inter-agir progressivement les deux types de lecture (Petitjean, 2001a; Rouxel, 2001 ; Langlade, 2001a).

86 Ce que confirme J.-L. Chiss (2011: 107) :

«Si en $6^{\mathrm{e}}$ les programmes privilégient des exercices classiques (questions, reformulations, résumé oral des lectures faites en classe), la porte est ouverte, dès la $5^{\mathrm{e}}$, à des activités plus "culturelles" [...] compte rendu de lecture, manipulation du livre, balayage de la $1^{\text {re }}$ et de la $4^{\mathrm{e}}$ de couverture, présentation orale d'un ouvrage par un élève à la classe, affiche, bande-annonce, catalogue de sélections de livre, exposition collective sur un ouvrage...). La tenue de "journaux de lecture", l'émergence de "cercles de lecture" font partie intégrante de cette lecture cursive. "

Il est vrai, concernant les lectures en classe, que l'opposition entre lectures « analytiques » et lectures « cursives » ou « lecture en classe » et « lecture personnelle » (Jordy, 2009), recoupe en partie l'opposition entre lecture "ordinaire» et lecture " lettrée » et renvoie à des débats qui agitent les didacticiens de la littérature. Le prix didactique à payer pour que la lecture littéraire ne devienne pas une activité d'un autre 
temps est de prévoir un enseignement progressif de cette compétence, du primaire au secondaire. Ce qui implique un certain nombre de contraintes :

- ajuster au mieux que l'on peut les corpus des textes étudiés aux habilités maitrisées par les élèves tout en ne les enfermant pas dans les genres et les auteurs qui leur sont familiers. De ce point de vue, la conception patrimoniale du corpus et l'ordonnancement chronologique par siècle, certes nécessaires à une conscience historique de la littérature, entrent en contradiction avec la non moins nécessaire prise en compte de l'encyclopédie des lecteurs, et de leur compétence linguistique. Ce qui signifie qu'il importe au collège, voire au lycée, et en se rappelant que le professeur de français est certes un médiateur de la culture mais aussi un technicien de la lecture au service des autres disciplines;

- de fournir les références contextuelles (auteur, intentions communicationnelles, intertextes, etc.) nécessaires à la compréhension historique des textes. Cela se fait à l'aide de notes diverses mais surtout par la constitution de dossiers documentaires qui devraient accompagner toute lecture suivie.

Le goût et le plaisir de lire peuvent se développer en alternant les régimes, les postures (Bucheton, 1999), les modes de lecture et en opérant une "dialectique » entre des activités "relevant de la participation » et d'autres "privilégiant la distanciation » (Dufays, 2005). Cela passe par des moments d'immersion dans le monde du texte, au fil d'une lecture "courante », « investie », et " oublieuse ». Cela nécessite aussi le recours à des activités liées à une métalecture impliquant des moments de réflexion, de mémoration, d'associations, de comparaisons, etc. Ils sont d'autant plus indispensables qu'un texte de fiction étant nécessairement "narcotisé » sous la forme d'un "monde extensionnellement incomplet», c'est avec l'aide de l'enseignant que les élèves pourront se livrer à un «travail d'élaboration du sens » et de colmatage des implicites ou de clarifications des connotations. Sans sous-estimer les différences statutaires entre le professeur et le groupe classe, il importe d'établir une "maïeutique interactive » et, comme l'écrit Y. Citton, de

«faire ensemble des hypothèses sur ce que dit le texte - sans le dire, dans la zone d'indétermination où il suggère sans affirmer, où il implique en nous laissant le soin d'expliciter ce que cela peut "vouloir dire". Dans une telle discussion, on n'exprime pas (directement) ses opinions, mais son observation du texte, lequel sert de terre commune où l'on peut retrouver ensemble, en dépit de nos diversités d'opinion, de présupposés et de valeurs ».

89 À l'encontre du fatalisme sociologique des uns ou du légitimisme culturel des autres, B. Lahire a bien montré que l'opposition, au niveau des modes de lecture littéraire, entre posture de type éthico-pratique et formalo-esthétique ne recouvre pas la partition lecteurs populaires/lecteurs lettrés, mais renvoie plutôt à l'opposition entre lecteurs professionnels (écrivains, enseignants) et lecteurs profanes. Il rejoint la distinction qu'opère V. Jouve (1993) entre «lecture avertie » et "lecture naïve » ou R. Rorty (1992) entre «lecture méthodique» et «lecture inspirée». Si le lecteur faiblement diplômé privilégie une lecture de type éthico-pratique (Burgos, 1992, 1993), le professionnel, quand il n'est pas en situation d'exercer sa pratique, en fait tout autant. Ce qui signifie qu'il s'agit moins d'opposer deux types scolaires de lecture (analytique et cursive) dont l'une serait plus exigeante que l'autre mais de chercher à développer les capacités interprétatives des élèves en distanciant leur rapport immédiat aux textes. Ce dont rend compte U. Eco (1985) en distinguant l'interprétation sémantique, qui «résulte du procédé par lequel le lecteur, placé devant une manifestation linéaire du texte, la remplit d'un sens donné ", et l'interprétation critique, 
qui est une "activité métalinguistique visant à décrire et à expliquer pour quelles raisons formelles un texte donné produit une réponse donnée ». Tout lecteur ordinaire, aussi «faible lecteur" soit-il, est nécessairement capable d'une interprétation sémantique car dès l'instant où « le lecteur impliqué prend corps dans le lecteur réel » (Ricœur, 1985) s'opère un acte de reconfiguration qui dépend à la fois de la compétence linguistique de ce dernier mais aussi de l'univers encyclopédique et culturel de son expérience et de son degré de familiarité avec les conventions esthétiques et le contrat générique du texte. Par contre, c'est dans l'espace scolaire, selon les situations interlocutives dans lesquelles il est placé, qu'il soit amené à justifier sont intérêt pour un livre lu ou sommé de l'expliquer, que le lecteur, en l'occurrence l'élève, doit être capable de défendre une interprétation critique. Pour ce faire, comme le veut la tradition de la philologie herméneutique, on lui conseillera de chercher à déterminer le sens de l'œuvre en ayant recours à l'intention de l'auteur, elle-même déterminée par les éléments du contexte de la production du texte (biographie, commentaires auctoriaux, état de la langue, horizon générique...). L'exercice n'a rien d'évident car, comme en apporte la preuve la génétique des textes (De Biasi, 2011) ou les historiens du livre (Chartier, 2008), les textes sont des réalités instables, sujettes à des variations génétiques et soumises à des décisions éditoriales. Ce qu'Y.Citton appelle la "détextualisation ». J'ajouterai que la transmission de savoirs latéraux externes n'est pas aisée en situation scolaire et s'il est vrai qu'interpréter un texte c'est nécessairement imputer ce qui est écrit à une instance énonciative, cela ne signifie pas qu'il faille utiliser le dessein ou l'intention de l'auteur comme seule norme acceptable d'interprétation. Cela est d'autant plus problématique que les enjeux communicationnels inhérents à toute production d'un texte, sont difficiles à théoriser, même si l'on ne manque plus de notions en ce domaine (posture, vision, registre, dispositions intentionnelles). À propos de ces dernières, si l'on en croit les pragmaticiens (Grice, 1979; Searle, 1985 ; Sperber \& Wilson, 1989), au cours d'un échange verbal, «il existe une propriété unique - la pertinence - qui détermine quelle information particulière retiendra l'attention d'un individu à un moment donné. " Je cite encore D. Sperber et D. Wilson : "Communiquer par ostentation, c'est produire un certain stimulus afin de réaliser une intention informative avec, en outre, l'intention communicative de rendre mutuellement manifeste au destinataire et au communicateur que le communicateur a cette intention manifeste ».

90 Ce bon fonctionnement des échanges présuppose, outre un savoir (général ou circonstanciel) partagé par les deux interactants, qu'ils sachent évaluer l'ajustement des énoncés au contexte. Ce n'est pas toujours simple pour les échanges ordinaires mais encore plus complexe pour les fictions littéraires. En effet, elles correspondent à une communication différée dont les buts sont souvent indécidables - à des degrés moindres pour le roman "à thèses » (Suleiman, 1983), la littérature dite " engagée " (Denis, 2000) ou ce que Ph. Hamon (1973) appelle le « discours contraint » du roman réaliste. Compte tenu de la présence d'une multitude de voix et du fait que les textes survivent à la disparition du contexte de leur production, cela signifie qu'il n'est certes pas nécessaire de renoncer à l'intentionnalité (il y a bien un auteur qui se dit à travers son discours) mais que, comme l'écrit J.-M. Schaeffer (2011: 93), l'intention de l'auteur est enchâssée dans la compréhension comme acte: "Ce que nous lisons, nous le saisissons, sans autre forme de procès, comme "ce qu'a voulu dire", et, que pour autant que notre acte de lecture donne naissance à une compréhension cohérente pour nous, la signification en question vaut pour comme signification de l'auteur ». 
91 Il reste que le texte ne résulte pas seulement d'un vouloir dire mais possède, par sa cohérence, une "capacité régulatrice" du sens qui oriente nécessairement les inférences du lecteur. Ce qui amène U. Eco à parler d'intentionnalité textuelle. En fait, comme le suggère J.-M. Schaeffer (ibid., 102), un texte ne saurait avoir une intention mais son pouvoir de signifier résulte de l'articulation dialectique entre deux intentionnalités : «En tant que structure intentionnelle, un texte est, en effet à la fois ce qui est causé par le vouloir dire d'un auteur, qui s'y exprime, et à partir de quoi chaque lecteur va construire ce qui de facto sera sa signification - à savoir le résultat de son activité de compréhension ».

On retrouve ici la distinction que fait R. Barthes (1972) entre ces deux intentionnalités que sont le «pour soi » de l'auteur et le «pour moi » du lecteur ou le modèle de la «triple mimésis » de P. Ricœur qui distingue mimésis 1 (le vouloir dire de l'auteur), mimésis 2 (l'incarnation de ce vouloir dire dans une structure textuelle ») et mimésis 3 (la réactivation de cette structure par le lecteur). On rejoint aussi la réflexion de F. Rastier (1989) pour qui le sens n'est ni entièrement dans le texte ni totalement dans l'acte de lecture, mais dans l'interaction entre les deux. U. Eco (2013 : 43) le rappelle : «Quand on a un texte à questionner, il est hors de propos d'interroger l'auteur. En même temps, le lecteur ne peut choisir n'importe quelle interprétation fondée simplement sur sa fantaisie : il doit s'assurer que le texte, d'une façon ou d'une autre, légitime et même encourage une lecture particulière. [...]. En d'autres termes, (il y a une) dialectique entre les droits des textes et les droits de leurs interprètes ». Ce que confirme A. Compagnon (2008: 194) qui, à la conclusion de son chapitre sur « Le lecteur » écrit :

«Une fois de plus, entre les deux thèses polaires qui ont pour elles une certaine consistance théorique, mais qui sont manifestement excessives et intenables l'autorité de l'auteur ou du texte permet d'instituer un discours objectif (positiviste ou formel) sur la littérature, et l'autorité du lecteur, d'instituer un discours subjectif - toutes les positions moyennes semblent fragiles et difficiles à défendre. [...] Mais pratiquement, nous vivons (et nous lisons) dans l'entre-deux. L'expérience de la lecture, comme toute expérience humaine, est immanquablement une expérience double, ambiguë, déchirée: entre comprendre et aimer, entre la philologie et l'allégorie, entre la liberté et la contrainte, entre l'attention à l'autre et le souci de soi."

En conséquence, il est de notre responsabilité de multiplier les exercices qui développent les capacités inférentielles et interprétatives des élèves et de montrer aux apprentis lecteurs que la validité de l'interprétation se mesure aux types et aux nombres d'aspects du texte dont elle est capable de rendre compte en fonction des contraintes déterminées par le texte. D. Olson (1998) le souligne : «Penser le texte exige $\mathrm{du}$ lecteur qu'il apprenne comment on peut interpréter un texte de différentes manières et qu'il détermine et mette en œuvre l'interprétation la plus pertinente, en fonction des indices que le texte lui propose. La lecture critique consiste à reconnaitre qu'un texte peut être compris de plusieurs façons, puis d'en tirer les conséquences en fonction des preuves apportées par le texte. »

Il reste qu'il y a des niveaux d'interprétation et, comme l'écrit J. Molino (1989 : 48-49) :

«Pas plus que les stratégies de fabrication, les stratégies de réception ne sauraient servir de norme d'interprétation (certes) du côté de l'interprète, aucune barrière ne saurait être mise pour limiter la liberté du commentaire. Interpréter, c'est toujours, selon la formule d'Heidegger, faire violence au texte et rien ne fixe les bornes de cette violence. [...] C'est ici qu'apparait un nouveau personnage, l'analyste. Fondamentalement, ce dernier est du même côté que l'interprète, du côté de la 
réception - de l'esthétique - mais son attitude est différente : il cherche non à interpréter le texte mais à en rendre compte. Se produit alors le décrochage entre interprétation et analyse [...]. Et ce décrochage se manifeste en ce que j'ai non seulement à étudier le texte, mais aussi les stratégies de production qui lui ont donné naissance et la diversité des interprétations auxquelles elles ont donné lieu. »

On retrouve cette même opposition chez J.-M.Schaeffer (1996: 164) qui fait la distinction entre "conduite esthétique » et «investigation savante » non pas sur le mode d'une opposition radicale mais en termes de niveau de traitement cognitif :

"Dans la mesure où la relation cognitive "basique" est indéniablement première par rapport à l'investigation scientifique et plus généralement savante, on peut expliciter la différence en termes de niveaux d'activité "cognitive" : on dira que l'investigation savante d'une œuvre d'art, de même que l'investigation scientifique du monde naturel sont des activités de niveau 2, alors que la saisie cognitive de ces mêmes objets dans la vie de tous les jours met en œuvre une relation cognitive de niveau $1 »$.

Passer du statut d'« interprète » à celui d'« analyste » nécessite la mise en place de protocoles du type de celui que propose Y. Reuter (1992) à propos d'une nouvelle de Pascal Mérigeau. Il consiste à faire réfléchir les élèves aux indices textuels sur lesquels ils s'appuient pour justifier leurs lectures (« réaliste-psychologique » ou "policière ») d'un même texte.

F. Rastier (2013: 196) le rappelle : « Les chemins de l'interprétation demeurent ainsi des chemins d'enquête qui varient avec les moments et les tâches et relèvent de diverses campagnes de lecture qui ont chaque fois un objectif différent et une entrée textuelle différente ".

Se pose néanmoins la question de savoir s'il existe de bonnes ou de mauvaises interprétations d'un texte et si le professeur est forcément le détenteur de l'unique clé interprétative. Reconnaissons avec C. Kerbrat-Orecchioni $(1980: 182)$ qu'il y a sûrement de « mauvaises lectures »,

« celle qui, par exemple, consiste à infliger à n'importe quel texte un traitement paragrammatique arbitraire et qui, faute de parvenir à endiguer les débordements $\mathrm{du}$ sens, aboutit par des voies opposées au même résultat que la lecture monologique: la négation du texte. Car si l'on peut lire n'importe quoi sous n'importe quel texte (et il serait facile de démontrer que tout texte soumis à une lecture paragrammatique incontrôlée, devient infiniment polysémique), alors tous les textes deviennent synonymes, et leur matériau signifiant indifférent. Lire ce n'est ni se soumettre corps et âme à la tyrannie des codes émetteurs, mais ce n'est pas non plus donner libre cours aux caprices de son propre désir/délire interprétatif.s ».

Ce dont témoigne aussi M.-L. Elalouf (2009) quand elle constate que l'insuffisante connaissance des formes grammaticales par les étudiants qui se destinent pourtant au professorat de Lettres, est source de contresens quand ils sont amenés à expliquer des textes poétiques. On sait combien les élèves connaissent le même type de difficultés.

Pour se prémunir de ce danger, l'autorité interprétative (qu'elle provienne du manuel ou du professeur) est un rempart nécessaire. À condition, cependant, de savoir écouter et entendre les réactions des élèves et de rendre acceptable la part subjective inhérente à toute lecture. Pour ce faire, comme le souligne V. Jouve (2003), il importe de respecter trois principes :

«1. [...] ne pas contredire les données objectives du texte (comme) les données factuelles qu'on n'a pas le droit de négliger. 
2. [...] annoncer le code de lecture utilisé (ce qui) revient à définir sa méthode et sa démarche en acceptant les limites et la relativité de toute analyse.

3. [...] respecter la logique du code utilisé [car] c'est respecter l'instrument d'analyse ».

101 C'est pourquoi l'enseignant incite les élèves à expliciter les arguments qu'ils utilisent pour justifier leurs interprétations premières, soit qu'il les confirme, soit qu'il les confronte à d'autres interprétations dont il explicite les présupposés. Le retour réflexif est important car il permet d'amener les élèves à prendre conscience des stratégies qu'ils utilisent et de transformer leurs connaissances déclaratives en connaissances procédurales (par exemple: prendre garde aux indices de généricité, prendre l'habitude, pour régler des problèmes de compréhension, d'inférer à partir du contexte, utiliser le bon dictionnaire, consulter un référentiel ou relire un passage, etc.). L'intérêt d'expliciter les connaissances déclaratives est que cela oblige les élèves à exercer leur métacognition (ne pas oublier de...) jusqu'à ce que ces connaissances deviennent inconscientes et routinisées. On sait, ici, l'importance des questionnaires ou des entretiens qui interrogent les élèves sur leurs représentations en matière de lecture, leurs attitudes face à la lecture, leurs goûts et réactions de lecteurs ou les accompagnent avec une visée formative dans leurs lectures (Nonnon, 1992). En ce sens, il ne suffit pas, comme le propose G. Langlade (2004a), de rappeler la part subjective inhérente à toute lecture et de faire "l'apologie du braconnage littéraire ». La valorisation de l'élève en tant que "sujet interprète » dans les textes officiels de 2002 pour le primaire (voir Bishop, 2009) ou de 2009 pour les lycées professionnels (voir A. Belhadjin, 2009) ne devrait pas omettre de souligner qu'il importe d'échelonner tout au long de la scolarité - du primaire au lycée - ces activités. Elles présentent un double intérêt. D'une part, ne pas réduire les apprentissages formels de la lecture à des tâches du type "répondre à des questions posées » et au lycée à la pratique des exercices canoniques que sont la dissertation et le commentaire. D'autre part, ne pas encourager les dérives interprétatives qu'autorise l'accent mis sur les lectures singulières. Il semble plus judicieux, tout au long de la scolarité, sous la forme de séquences d'enseignement, impliquant lecture et écriture, de permettre aux élèves de prendre conscience des paliers d'organisation du sens dans les textes et de la pluralité possible des lectures d'un même texte. À titre d'exemples, on se reportera à la réflexion de J.-L. Dufays (1997) et aux activités qu'il décrit à propos de la "lecture plurielle» au lycée. On tiendra compte aussi des propositions de J.-M. Privat (1997) en matière de lecture ethnocritique en dernière année du collège ainsi que des analyses de type ethnocritiques de F. Menand-Doumazane (2011) et de M. Laparra (2010, 2011). Ce qui n'empêche pas qu'il faille s'interroger sur le statut épistémologique de la critique littéraire et des enjeux didactiques du commentaire de texte comme le fait N.Cordonier (1990) dans son analyse des gloses savantes de Chantre d'Apollinaire.

\subsection{De l'immanentisme structural à la linguistique textuelle}

Qu'elles aient subi la concurrence des modèles générativistes (le texte comme productivité qui change les règles) ou l'influence des théories bakhtiniennes (le texte comme "espace dialogique»), les théories immanentes des années 70 vont être concurrencées par la linguistique textuelle, les théories de l'énonciation, la pragmatique et l'analyse de discours (pour une synthèse de l'évolution de ces différents courants, et plus particulièrement de la linguistique textuelle, on se reportera à 
Charolles \& Combettes, 1999 ; Combettes, 2010 ; Adam, 2010). Il est indéniable que Pratiques, en particulier par l'intermédiaire de travaux de B. Combettes, de M. Charolles et de J.-M. Adam - voir leurs nombreux articles et les numéros consacrés aux plans d'organisation des textes (Pratiques, $n^{\circ} 57$ ), à la cohésion textuelle (Pratiques, $n^{\circ} 85$ ) aux types et aux genres de textes (Pratiques, $\mathrm{n}^{\circ} 56$ ) - a joué un rôle majeur dans ce domaine. J.-M. Adam, depuis son livre en collaboration avec J.-P. Goldenstein (1976) jusqu'aux plus récents écrits avec U. Heidmann $(2009,2010)$, s'est toujours intéressé à la littérature et rappelle inlassablement que les œuvres littéraires sont des configurations langagières et discursives. Il le prouve en se consacrant à différents genres du littéraire, que ce soit la poésie (Adam, 1985), le théâtre (Adam \& Leclerc-Adam, 1988) ou les différentes formes du récit (Adam, 1984, 2011).

Il serait fastidieux d'énumérer les numéros de Pratiques qui ont contribué au développement de la linguistique textuelle selon les genres fictionnels. Par l'intermédiaire d'activités au long cours (écritures de textes complets, par exemple), consacrées à différents genres de la fiction, il s'est agi d'associer lecture et écriture et d'apporter des savoirs linguistiques et textuels selon les besoins des élèves et les problèmes qu'ils rencontrent tant au niveau de la lecture que de la production de textes. Je ne retiendrai que quelques articles qui, dans une perspective praxéologique et à l'aide de démarches et d'exercices appropriés, ont apporté la preuve de leur efficience didactique. À titre d'exemples, pour le théâtre, A. Petitjean (1984), D. Coltier (1992), D. Bessonnat (2003); pour la poésie, J.-F. Halté (1978), J.-P.Goldenstein (1978a), K. Canvat et G. Legros (1997), R. Michel (1997). Le récit (romans ou nouvelles), en écho à la doxa scolaire des années 70, a fait l'objet d'études consacrées à des genres ou des sous-genres: C. Schnedecker (1990) pour le portrait; K. Canvat et C. Vanderdorpe (1996) pour la fable; M.-C. Kirpalani (2000) pour une poétique de la nouvelle; C. Amstutz (1993); C. Vernet (1995) pour les nouvelles policières; O. Dezutter (2002) pour l'épistolaire; C. Masseron (1990) pour les nouvelles fantastiques. Les travaux ont aussi porté sur des formes d'organisation textuelle: les scènes romanesques (Reuter, 1994 ; Cadet, 1994 ; Canvat \& Dufays, 1994) ; les désignateurs et les chaines de référence pour la textualisation des personnages (Masseron \& Schnedecker, 1988) ; les formes et les fonctions des dialogues (Bessonnat, 1990 ; Coltier, 1989) ; les formes et les fonctions de la description (Reuter, 1987 ; Vinson, 1987), etc. Ce renouvellement des enseignables s'est accompagné d'une réflexion portant sur les démarches d'enseignement. En témoigne l'intérêt de la notion de "séquence » sous la forme d'une suite d'activités progressives, planifiées et finalisées par un objectif général d'enseignement/ apprentissage et diversifiant les tâches et les supports, illustré par nos collègues de Genève (Dolz, Rosat \& Schneuwly, 1991) et par Pratiques à diverses reprises (Masseron, 1996 ; Perrin, 1996).

104 Il est indéniable que l'approche immanente des textes a provoqué une reconfiguration progressive de la discipline dans la mesure où les théories formelles ont eu une influence certaine sur les contenus à enseigner (voir les attendus de l'épreuve de stylistique du CAPES) ou la matière des manuels) et surtout sur la conception de la lecture des textes littéraires. Mais il faut prendre acte, ici, des écarts existant entre les élaborations didactiques proposées dans nombre d'articles de Pratiques et ce qu'elles deviendront quand elles seront transposées sous la forme de l'exercice baptisé la « lecture méthodique ». Il a été modélisé par les textes officiels (BO de 1987 et 1988) et relayé par les revues (voir le $n^{\circ} 90$ du Français aujourd'hui), certains ouvrages de la 
collection B. Lacoste (Descotes, 1989 ; Weiland \& Puygrenier-Renault, 1997) avant d'être généralisé par les manuels. Les partisans de la lecture méthodique ne manquent pas d'affirmer, comme le fait M. Descottes (1990) que l'acte de lire est « une activité de construction du sens mettant en jeu les opérations d'anticipation, d'élaboration d'hypothèses de sens " et qu'il faut partir des "représentations» des élèves, pour ensuite les confronter aux textes avec la médiation des savoirs procurés par l'enseignant. Tel est l'esprit de la pédagogie de la "séquence " (Armand, Descottes, Jordy \& Langlade, 1992) qui, à travers les " séances » organisées, mêle différents types de lecture dont des «lectures méthodiques». En fait, si on en juge par nombre d'ouvrages édités par les centres régionaux de documentation pédagogique (CRDP), les séquences sont formelles et la compréhension du texte est réduite à la maitrise de connaissances linguistiques et sémiotiques. C'est ainsi, pour ne prendre qu'un exemple, que J.-P. Chanteau (1990) limite l'étude, en sixième, du Mariage forcé de Molière à des exercices formels: "lecture du titre, lecture de la liste des personnages, lecture tabulaire, lecture du tableau des substituts lexicaux, lecture de la scène d'exposition, construire les schémas actantiels, dégager la structure narrative, relever les apartés et les monologues ». On sait aussi les difficultés éprouvées de travailler en séquence (au moment de leur préparation comme de leur mise en œuvre) analysées par M. Ayraud et J.-P. Benoit (1996), au point que les textes officiels de 2008 en ont supprimé le principe. Quant à la lecture méthodique, Pratiques en dénoncera très tôt les méfaits didactiques (Michel, 1998) avant que la critique ne soit relayée par les didacticiens de la littérature (Langlade, 2001b; Rouxel \& Langlade, 2004a) au nom d'une prise en compte « de la dimension subjective de la lecture » et " des réalisations effectives des sujets lecteurs ». Plus récemment, l'opposition à l'exercice est venue des partisans des humanités (voir le numéro 135 du Débat, 2005) et plus particulièrement de P. Laudet (2009). Ce dernier, inspecteur général des lettres, se livre, au cours d'un séminaire académique daté du 17 mars 2009, à une véritable charge contre les pratiques de l'explication de textes en vigueur au secondaire :

«Loin d'être seulement relativisée par les proportions excessives prises par la littérature de jeunesse, les grands textes de la littérature ont pu l'être aussi par les approches trop exclusivement formelles auxquels ils ont donné lieu. La dérive techniciste n'était d'ailleurs pas tant dans les textes officiels antérieurs qu'elle ne s'est installée peu à peu dans les classes. Que de passages magnifiques réduits à des manipulations formelles, aléatoires, dénuées de sens et d'enjeux. L'ambition des nouveaux programmes est de corriger le technicisme ambiant dans les classes par un souci plus grand d'humanisme ("fonder une culture humaniste"). Faut-il que toute séance d'étude de texte finisse dans une grille ? Chaque texte doit-il passer au lit de Procuste du tableau énonciatif? Puissent, en un sens, les nouveaux programmes faire souffler un vent révolutionnaire, et mettre un bonnet rouge au vieux schéma quinaire! Désengager les textes arraisonnés dans tous les schémas narratifs ou actantiels, au demeurant très utiles mais souvent désinvestis de toute valeur, c'est conjuguer à nouveaux frais "l'esprit de géométrie", à condition de ne pas tomber dans le seul cadastre, avec "l'esprit de finesse", dont le développement reste quand même un des objectifs majeurs de l'étude de la littérature. »

Avec le recul, on peut donc soutenir que la "dérive techniciste » dont il est question tient plus à l'insuffisante maitrise des connaissances linguistiques et des savoirs didactiques par les auteurs de manuels (voir Sitri, 2003 ou Coirault \& David, 2011) et par nombre d'enseignants, compte tenu de leur formation académique initiale, qu'aux théories elles-mêmes. C'est ainsi que la poétique et la narratologie françaises et anglosaxonnes, ont été réduites au schéma narratif ou à des problèmes de focalisation. Les 
grammaires de textes provenant des pays nordiques (T.A. Van Dijk, J.Petôfi, E. Werlich, E. Gülich, H. Isenberg...) ont engendré des typologies textuelles abstraites. Les travaux sur la cohésion et la cohérence textuelle (I. Bellert, M.A.K. Haliday et R. Hasan...) ont fini sous la forme de relevés de connecteurs ou de marqueurs textuels (pour un bilan, voir Achard-Bayle, 2010). Certes, il ne faut pas sous-estimer la part de responsabilité de certains travaux de didactique et surtout des Instructions officielles de 2000 distinguant pour les « activités grammaticales ", la « grammaire de texte ", la "grammaire du discours" opposées à la "grammaire de phrase ». J.-M. Adam (2010) reconnait que la première "est plus un obstacle méthodologique qu'un outil heuristique » et D. Maingueneau (1999) considère qu'il y a "bien des inconvénients à employer grammaire pour des phénomènes textuels et énonciatifs. »Censés apporter un intérêt didactique pour la production de texte, les phénomènes énonciatifs et transphrastiques (type d'attitudes élocutives, opposition des plans de texte, progression thématique, chaine de référence...) sont devenus inintelligibles quand ils ont servi à élaborer des grilles d'analyse des textes. La vigilance de Pratiques n'a pas toujours fait défaut en la matière et on trouvera dans E. Nonnon (1998) une mise en garde par rapport aux modes de transposition des théories du texte en formation des enseignants (changement du statut épistémique des théories de référence, formes de simplification des savoirs scolaires élaborés, modes d'appropriation par les élèves des savoirs enseignés). La "dérive techniciste " provient aussi d'une confusion entre les savoirs que devraient posséder les enseignants et ceux à transmettre aux élèves. Comme l'écrit T. Todorov $(2007: 23)$ :

«Il est vrai que le sens de l'œuvre ne se réduit pas au jugement purement subjectif de l'élève, mais relève d'un travail de connaissance. Pour s'y engager, il peut donc être utile à cet élève d'apprendre les faits d'histoire littéraire ou quelques principes issus de l'analyse structurale. Cependant, en aucun cas l'étude de ces moyens d'accès ne doit se substituer à celle du sens qui est sa fin. Un échafaudage est nécessaire pour élever un bâtiment mais il ne faudrait pas que le premier remplace le second : une fois le bâtiment construit, l'échafaudage est destiné à disparaitre. Les innovations apportées par l'approche structurale dans les décennies précédentes sont les bienvenues à condition de garder cette fonction d'outils, au lieu de devenir leur propre but ».

C'est pourquoi, il y a fort à parier que ce n'est pas en se faisant l'apôtre d'une approche hédoniste de la lecture (Pennac, 1992), ni en affirmant que ce qui s'enseigne au cours de français n'est "ni mesurable ni objectivable" (Grange \& Leroux, 2005), que l'on trouvera les solutions aux problèmes. Les propos tenus par P. Laudet (2011) sont à cet égard assez révélateurs de l'esprit régressif qui imprègne les textes officiels de 2008 :

« Tout texte, par son tissage complexe, est bien un tapis dont il faut chercher le motif secret. Son dessin ne saute pas immédiatement aux yeux et requiert une vigilance de l'intelligence [...], un art de lire au-dedans, entre les lignes. [...] Contre la raideur des lectures plaquées, retrouver donc la souplesse d'une authentique disponibilité du texte et d'une attention à sa singularité. [...] Lire n'est pas seulement la maitrise d'une compétence, mais aussi se disposer à recevoir la grâce d'un don".

C'est bien là revenir à « la religion du texte » (Kuentz, 1972) et à la pratique du " tête à texte " (Kuentz, 1974) dont on sait combien elles sont discriminantes sur le plan socioculturel. 


\section{Mise en perspective de la « lecture littéraire »}

\section{1. Écrire et produire des textes}

107 Ce serait une erreur de s'enfermer dans la seule problématique de la «lecture littéraire » sans mesurer, comme le rappelle B. Daunay (2007), qu'agir sur l'appétence des élèves pour la lecture des textes littéraires implique aussi que soit donnée aux élèves la possibilité de "faire» de la littérature et pas uniquement de la commenter. Postulat sacrilège pour les défenseurs des humanités qui, à l'instar de P. Clarac (1964), considèrent les textes littéraires comme des «témoignages sacrés » dont " professeurs et élèves ne doivent s'approcher qu'avec respect et comme en tremblant » et font de la littérature un modèle normatif $\mathrm{du}$ bon usage de la langue et de l'écriture (voir D. Sallenave, supra). Or, on sait (Dabène, 1987; Bourgain, 1989) combien de telles représentations sont sources pour les élèves d'«insécurité scripturale». Cette démystification salutaire du postulat du " génie » et de l'« inspiration », n'enlève rien à l'admiration que l'on peut porter à un auteur ou à une œuvre, bien au contraire. Il ne s'agit plus de cette « admiration pétrifiante, celle qui fait que l'on reste bouche bée face à l'œuvre sacralisée », comme l'écrit V. Houdart-Merot (2004c: 230) mais d'une «admiration irrespectueuse et créatrice, celle qui se transforme en écriture ». Sans confondre illusoirement ou avec démagogie pratiques des élèves et "métier de l'écrivain ", il s'agit de rendre les lectures des élèves créatrices et leur écriture productive. Cette ambition a été très tôt assumée par Pratiques, sous l'autorité de R. Barthes (1975 : 19). En effet, ce dernier, au cours d'un entretien, nous confiait : « Il faudrait donner aux enfants la possibilité de créer des objets complets (ce que le devoir ne peut être), dans une temporalité longue. Il faudrait presque imaginer que chaque élève va faire un livre et qu'il se pose toutes les tâches nécessaires à sa réalisation ».

C'est ainsi que, dans Pratiques, après avoir décrit les attendus et les limites de la rédaction traditionnelle (Pratiques $n^{\circ} 26$ et $n^{\circ} 29$; Charolles et al., 1989), nous avons œuvré à l'expérimentation d'activités d'écriture dites «longues » ou « en projet » au niveau du collège (Halté, 1985 ; Petitjean, 1982, 1994 ; Masseron, 1994). À propos des dites activités, J.-F. Halté (1987) pouvait écrire qu'elles permettent de transformer la classe et les élèves en "une communauté des apprentis (scripteurs), sous leurs différentes instanciations (d'écrivains, de public, de critiques, de théoriciens ». Ce n'est pas le lieu, ici, de développer l'apport de Pratiques et de nombreuses recherches en matière de « didactique de l'écriture » (Pour une synthèse, voir Barré-de Miniac, 1995 ; Reuter, 1996b) et l'élaboration successive de différentes conceptualisations du « modèle rédactionnel » et de la « compétence scripturale » proposées par la revue. Je retiens des différentes expériences que j'ai pu moi-même mener en collège, en formation et plus tard à l'université qu'il est possible de modifier profondément les rapports des élèves, comme des enseignants, à la littérature. Cela peut prendre aussi la forme de «jeux d'écriture » (Idt, 1975 ; Duchesne \& Leguay, 1984 ; Carré \& Debysser, 1978), de « jeux poétiques » (Goldenstein, 1978 b; Delas, 1983), de la pratique de la "réécriture » (Ricardou, 1978; Oriol-Boyer, 1988; Pratiques, $\left.n^{\circ} 105-106\right)$ et surtout des «ateliers d'écriture » (Pratiques, n 61 et 89, Bisénius-Penin, 2005 ; Monte, 2012; Oriol-Boyer \& Bilous, 2013). Écrire avec, dans ou contre un texte littéraire, multiplier les ravaudages scripturaux, imiter, emprunter, transposer, bref entrer dans "une coopération scripturale » concerne tout autant la lecture que l'écriture et augmente l'expertise des élèves en les incitant à se poser des questions tant éthiques, linguistiques 
qu'esthétiques. Comme l'écrit A. Rabatel (2006: 56), l'élève peut plus facilement "adopter une posture lectorale qui est à la fois dedans et dehors, empathique et distanciée [...]. Qu'aurais-je fait à la place des acteurs, de l'auteur - aurais-je sélectionné les mêmes éléments, adopté la même organisation du discours, la (les) même(s) perspective(s), le(s) même(s) registre(s), quels effets résulteraient de tel(s) ou tel(s) changements de plan d'énonciation, de référenciation ou nominale ».

Acceptées par l'institution au niveau du primaire et du collège, ces «pratiques d'écriture " se heurtent au lycée et surtout à l'université à l'hégémonie de la "rhétorique du commentaire" même si des avancées indéniables existent. (voir Houdart-Merot \& Mongenot, 2013 ; Bisénius-Penin, 2013a, 2013b ; et la thèse d'A.M. Petitjean, 2013, sur les pratiques créatives d'écriture littéraire dans les universités). C'est ainsi que l'on doit aux IO de 2000 pour les lycées un retour des exercices d'invention. Ils ont fait l'objet de débats et de polémiques dont témoignent en particulier S. Plane (2001), B. Daunay (2003), V. Houdart-Mérot (2004b), N. Denizot (2005), A. Petitjean (2005) ainsi que les numéros 127 du Français aujourd'hui (1999), 57 d' Enjeux (2003) et 127-128 de Pratiques (2005). J'ai déjà exprimé mes réticences à l'égard des formes prises par l'exercice tel que le législateur l'a formaté en tant qu'épreuve du baccalauréat et redis, par contre, l'intérêt de l'activité en situation formative. On peut lui attribuer, comme je l'ai montré (Petitjean, 2005), une double fonction : s'approprier et restituer les connaissances littéraires, qu'elles soient formelles ou historiques, et développer l'expression et la créativité. En permettant aux élèves d'échanger leurs expériences de lecture et d'écriture, qu'elles soient scolaires ou extra-scolaires (Penloup, 1999 ; Fabre, 1993), on multiplie les occasions pour les élèves de donner sens et cohérence à leur réalité quotidienne, qu'ils souhaitent s'en échapper ou éprouvent le besoin d'investir dans leurs lectures, comme dans leurs écrits, leurs problèmes d'adolescents (voir Huyhn, 2004 ; Legoff, 2004). Il y a là de quoi entrer en débat avec la pédagogie Freinet telle qu'elle a pu être exportée au secondaire (Clanché, Debarbieux \& Testanière, 1994), comme l'a montré M.-F. Bishop (2005).

\subsection{Les théories-psychocognitives}

110 Il est assez étonnant de constater à quel point les recherches en didactique de la « lecture littéraire » font l'impasse sur les théories cognitives et psycholinguistiques de la lecture qui se sont développées dans les années 80 (Denhière, Legros, 1983 ; Pagé, 1985 ; Thérien \& Fortier, 1985 ; Smith, 1975 ; Pratiques, n 35) et ont continué depuis (Giasson, 1990 ; Fayol et al., 1992 ; Coirier, Gaonac'h \& Passerault, 1996; Colder \& Gaonac'h, 1998 ; etc). On se reportera aussi, pour un état de l'art des théories $\mathrm{du}$ «traitement cognitif du récit », à J.-M. Schaeffer (2010) et pour une analyse des difficultés de compréhension des «faibles lecteurs » à R. Goigoux (2000) et L. SprengerCharolles et P. Colé (2013). Le fait est d'autant plus regrettable que la théorisation du lien entre "compréhension » et " interprétation » (Reuter, 2001) demeure insuffisante (voir, par exemple, les deux opérations telles qu'elles sont mentionnées avec profusion et de façon aléatoire dans les Instructions pour le primaire de 2002). On comprend mieux, à l'aide de ces recherches, les processus par l'intermédiaire desquels se construisent les significations d'un texte au cours des actes de lecture. 
On peut considérer que lire un texte implique :

- de posséder des connaissances linguistiques (lexique, syntaxe, super-structures textuelles) ainsi que des connaissances encyclopédiques et référentielles par rapport au domaine de référence du texte ;

- de savoir combiner (et naviguer entre) des opérations de niveaux différents, de manière ascendante (des plus petites unités linguistiques vers les unités supérieures, par compréhension locale d'informations explicites et par inférence en intégrant des informations non explicites) et de façon descendante (des macrostructures vers les unités plus petites en formulant une interprétation globale du texte qui permet de résoudre des difficultés de compréhension locales) ;

- d'être capable, grâce à sa familiarité avec le genre textuel, d'élaborer à partir des amorces textuelles des représentations mentales virtuelles, progressivement construites, flexibles et évolutives.

112 Concernant les textes de fiction, elles s'appliquent aussi bien à des existants personnifiés, des lieux et des actions, des tensions psychologiques, des conflits axiologiques, selon une intentionnalité signifiante. Le lecteur aura d'autant plus d'aisance dans sa lecture qu'il est capable d'adopter avec plasticité l'identité sémantique des différents personnages présents dans l'univers de la fiction ou de privilégier l'un d'eux, à l'ethos, au logos et au point de vue duquel le lecteur s'assujettit dans une posture d'identification.

113 Après D. R. Olson (1998), C. Tauveron (2009: 64-65) distingue différents types d'opérations cognitives qu'implique l'acte de lire :

«Dans l'ordre du nécessaire au quasi nécessaire

1. Les déductions (à partir de présupposés qui conduisent à " penser que c'est »;

2. Les inférences pragmatiques et encyclopédiques qui conduisent à "penser que cela doit être".

Dans l'ordre du conjectural

1. L'élaboration d'hypothèses interprétatives locales (sur l'intrigue) qui conduisent à "penser que cela pourrait être" ;

2. La détermination de la stratégie narrative et de la valeur d'illocution qui conduisent à "penser que cela pourrait vouloir dire".

Nous avons ajouté que le lecteur, dans le souci d'opacifier le texte maintenant compris pouvait re-problématiser le texte et ré-accomplir le parcours, à partir d'une sélection différente d'indices. »

114 Ces opérations cognitives, qu'elles soient convergentes ou divergentes, selon des processus analytiques ou analogiques, conscients ou inconscients, s'accompagnent de jugements (pertinence, possibilité, vraisemblance, reconnaissance ou révélation, etc.) ou d'évaluations d'ordre éthique ou esthétique.

115 Il convient donc de poursuivre au collège, voire au lycée, les exercices portant sur les inférences, le colmatage des implicites, les ambiguïtés sémantiques, les significations connotatives, etc. (voir Cèbe et al., 2012) en multipliant, comme le propose A. Godard (2011 : 52), des «types de lecture allant du rapide survol (skimming) ou feuilletage non sélectif, à la lecture par balayage (scanning) pour chercher une information précise, ou une lecture-action, attentive aux procédures dans la lecture de consignes, d'un mode d'emploi ou d'une recette jusqu'à la lecture critique ». 


\subsection{Textes et valeurs}

116 La question des valeurs à propos des textes littéraires et des œuvres d'art plus généralement dépend pour une large part du point de vue que l'on adopte (historique, sociologique, philosophique, esthétique, sémiotique, linguistique) pour y répondre. Il ressort de ces différents paradigmes théoriques qu'il existe des ordres de valeurs hétérogènes que l'on mobilise holistiquement dans la relation que nous entretenons avec les textes. On peut ainsi apprécier ou disqualifier un texte en fonction de valeurs éthiques (morales, religieuses, politiques, sociales) ou esthétiques. Ces dernières sont elles-mêmes non homogènes puisqu'elles sont afférentes tant au domaine de la technicité (reconnaissance de l'habilité opérale de l'auteur) qu'au degré de satisfaction et à la charge émotionnelle que procure l'œuvre. Ces ordres de valeurs peuvent potentiellement entrer en conflit comme l'attestent les querelles littéraires ou le fait qu'un auteur (je pense à Céline) peut être apprécié pour son écriture mais disqualifié pour les contenus véhiculés par certains de ses textes. On peut compléter ces deux modes de valorisation des textes par une valorisation historique qui correspond aux jugements d'expertise émis sur la base de connaissances latérales qui permettent d'apprécier un texte à l'aune des innovations opérées dans le contexte de sa production et des résonances qu'il connait dans l'histoire de sa réception. J'ajoute qu'il importe de faire la part entre les valeurs impliquées dans les textes et celles projetées par le lecteur. C'est pourquoi nous avons toujours été attentifs dans Pratiques, sans négliger pour autant les normes axiologiques des élèves, au problème de la " mise en texte des valeurs ». Dans un premier temps, elles ont été traitées essentiellement en référence aux modélisations sémiotiques d'inspiration greimasienne. C'est ainsi que J.-P Goldenstein (1976) relate son travail sur Mateo Falcone en classe de quatrième, au cours duquel, après avoir pris la mesure des « réactions affectives " des élèves à la lecture de la nouvelle, il focalise l'analyse sur le jeu d'oppositions entre les vraies valeurs du maquis familial que l'enfant trahit et celles citadines symbolisées par la "montre ", objet du désir convoité. De même, C. Masseron et B. Petitjean (1979), au cours d'un travail sur Germinal mené en classe de seconde technique et en troisième, font découvrir aux élèves de quelles valeurs le récit et plus particulièrement les personnages sont les porteurs. Par la suite, en s'appuyant sur les travaux de P. Hamon (1984) et de V. Jouve (2001), A. Leclaire-Halté (2003, 2004a) travaillera sur les dispositifs générateurs de valeurs dans un genre narratif inscrit au programme des classes de cinquième : les robinsonnades. Elle le fait avec l'objectif de permettre aux élèves, audelà du repérage des valeurs dans les discours, d'objectiver ces dernières, de participer à un débat argumentatif et de les rendre capables de formuler un jugement autonome (Masseron, 2003a). L'analyse des valeurs a aussi porté sur les foyers axiologiques tels qu'ils se déploient dans les textes non narratifs (voir le numéro 117-118, "Textes et valeurs ", dont Masseron, 2003b). Plus récemment, A. Leclaire-Halté (2010), à partir des échanges entre l'enseignante et les élèves à la suite de la lecture qu'elle leur a faite de l'album intitulé $V u$ à la télé de C. Desmarteau, examine comment s'opèrent les conflits entre les valeurs transmises par l'album et celles auxquelles adhèrent l'enseignante, d'une part, et les élèves, de l'autre. Comme l'a montré A. Rabatel (2004b, 2005), dans l'économie interne de la majorité des œuvres romanesques données à lire et à analyser, les valeurs sont « omniprésentes » mais souvent « opaques » car

«malaisément référables à une instance énonciative en cas d'enchâssements des instances ou de la polyphonie, surtout en contexte d'effacement énonciatif, peu 
fiables lorsque la source énonciative, pourtant dûment identifiée ne s'avère pas digne de confiance ou dans les cas hypothétiques de consonance ou de dissonance implicite par exemple lorsqu'on se demande si le narrateur épouse la vision du monde de ses personnages, si le sujet parlant (l'auteur) pense comme le narrateur (le locuteur). Le récit est donc problématique sous l'angle des valeurs parce qu'on n'est jamais sûr que le narrateur ne soit pas, en quelque manière, le complice des personnages négatifs et des valeurs contestables qui ne sont pas partagées par la doxa dominante ( $c f$. Flaubert, Zola, Wilde, Marcel Aymé et plus près de nous Houellebecq), malaisément assignables: où se trouvent les valeurs? Bien sûr, elles émergent dans le dire, l'être, l'agir des personnages ( $c f$. l'analyse de l'effet idéologie chez P.Hamon), mais est-on assuré d'avoir épuisé ainsi la liste des lieux d'émergence des valeurs ? Les structures narratives sont-elles neutres par rapport à l'éthique ou au contraire impliquent-elles une certaine représentation $d u$ monde?».

En fonction de quoi, il me semble judicieux, à l'encontre de la valorisation excessive du "sujet lecteur» («un texte littéraire ne peut véritablement exister que lorsqu'il est "produit" par le lecteur » affirme G. Langlade, 2004a, 2004b), de fournir aux élèves les rudiments d'analyse utiles pour se repérer dans le «maquis du sens et des valeurs». Certes, toute lecture est nécessairement subjective dans la mesure où tout texte de fiction est à des degrés divers indéterminé et narcotisé et que chaque lecteur projette dans le texte sa mémoire personnelle et les valeurs incorporées à sa socioculture. Il n'en demeure pas moins qu'il existe une perspective axiologique de l'instance énonciative et qu'il est opportun de faire la part entre les valeurs objectivables à partir des données textuelles et les valeurs subjectives investies par le lecteur. De ce point de vue, deux écueils sont à éviter, au niveau du collège et surtout du lycée. D'un côté, demander aux élèves de répondre à des questions auxquelles les spécialistes n'ont pas de réponses unanimes du type: que pense Molière de l'amoralisme de Dom Juan, comment concilier l'ethos anarchiste du narrateur du Voyage au bout de la nuit et celui de Céline par rapport à ce que l'on sait de l'individu Destouche? De l'autre, sous prétexte de rejet du technicisme dans l'étude des textes et d'un intérêt porté à la « relation intime entre l'élève et le texte » (Mathis, 2001), transformer la classe en un forum d'échanges d'opinions à propos de l'aspect sympathique ou non d'un personnage dont les élèves apprécient ou pas, d'un point de vue moral, la conduite. Il ne faudrait pas, comme un certain moralisme ambiant inclinerait à le faire penser, oublier que les textes littéraires ne sont pas soumis à des contraintes morales (voir les condamnations pluriséculaires des lectures et des lectrices de romans ou les agissements de la censure) et ne sauraient servir de modèles ou de contre-modèles. On y reviendra dans le numéro de décembre 2014 de Pratiques en préparation et intitulé « La morale en question(s) ». Ce qui signifie que bien que je partage la mise en garde de B. Daunay (2007) quand il écrit :

"Chez l'élève un rapport esthétique au texte [qui] passe, outre, les dimensions cognitives qu'institue un rapport distancié au texte, par la réévaluation de la part subjective dans l'approche des textes (où ne sont pas minorées les questions du goût, du plaisir, de l'émotion, des valeurs...) mais aussi celle de la part culturelle, où la dimension patrimoniale, sans être réifiée, est interrogée ".

118 Je demeure réticent à l'idée qu'il faille accorder la priorité au «jugement esthétique » des élèves dans l'approche des textes, et cela, pour au moins quatre raisons :

- on ne prend pas en considération le fait que les fictions littéraires scolarisées ont pour caractéristique d'être dominées par une intention esthétique et appartiennent à un «canon» (tel est le statut des «classiques») dont la valeur est présupposée par le geste 
même de canonisation. Ce qui signifie que les réactions de (dis)satisfaction des élèves face aux textes qui leur sont donnés à lire et à étudier ne manqueront pas de disqualifier ceux qui les formulent ;

- on laisse entendre que la relation esthétique, qui ne peut-être que subjective, est nécessairement frontale et binaire (j'aime ou je n'aime pas) alors qu'elle emprunte, comme le souligne B. Vouilloux (2006) « les voies de la triangulation symbolique » au sens où : « entre le sujet et l'objet, il y a toujours quelqu'un ou quelque chose : une tradition, une mémoire, des modèles, des prescripteurs, etc. Bref cet "entre" n'est autre que l'espace tiers ou médian par rapport auxquels sont définies les places du sujet et de l'objet et qui est donc un espace interprétant. De ce qu'un accord ne puisse s'établir aisément sur les propriétés de l'œuvre qui pourraient justifier le jugement que l'on porte sur elle, on ne saurait déduire que le seul jugement possible est celui qu'émet une subjectivité souveraine »;

- les descriptions des jugements subjectifs des élèves que proposent certains articles de didactique, sauf si elles permettaient d'ajuster la programmation des textes donnés à lire à leurs expériences de « jeunes » lecteurs - ce qui entrerait en contradiction avec la dimension patrimoniale de l'enseignement de la littérature - sont d'un intérêt relatif dans la mesure où elles ne font que confirmer les études sociologiques sur le sujet sans posséder la rigueur méthodologique de ces dernières ;

- si la valeur affective que les élèves accordent aux œuvres lues est pour une part essentielle liée à l'intensité des émotions qu'ils éprouvent en les lisant, il importe aussi de ne pas sousestimer les gains cognitifs qu'elles leur procurent et de leur montrer par quels procédés les textes représentent, expriment et suscitent ces émotions. On ne peut que regretter que les articles de didactique qui privilégient le " plaisir du texte littéraire », pour reprendre le titre du numéro 67 d'Enjeux, soient régressifs de ce point de vue et ne capitalisent pas les recherches antérieures.

119 Afin d'éviter de revenir à l'ineffable poétique, il serait judicieux que les recherches sur la « lecture littéraire " prennent en compte les nombreux travaux qui s'intéressent à l'ontologie de l'art, du point de vue de la philosophie de l'art (Dewey, 1997; Shusterman, 1999 ; Goodman, 1990 ; Pouivet, 1999 ; pour une synthèse, Lories, 1988) ou à la sémiotisation des valeurs dans les textes (voir Greimas, 1987 et l'École de Paris Fontanille, 1999; Geninasca, 1997). Je renvoie aussi à J.-M. Schaeffer (1996) ou G. Genette (1997) qui problématisent ce qu'ils appellent « une conduite esthétique » ou la « relation esthétique ». Je n'insiste pas sur les débats qui opposent les pragmaticiens et les philosophes analytiques - R. Michel (2005) en donne un aperçu - et retiens que les principaux apports de ces courants sont de contribuer à la théorisation de notions comme la «conduite esthétique», le «jugement de valeur» ainsi que celles d'« expérience », de " plaisir » ou de « satisfaction » esthétique. Elles mettent en débat les arguments qui opposent les objectivistes aux subjectivistes concernant la définition même de l'art et de ses artefacts ainsi que le statut de l'appréciation esthétique et méritent que l'on en tienne compte sur le plan didactique (voir infra, 4.2).

\subsection{Textes et histoire}

Dès les années 70-80, on assiste à un renouvellement en profondeur de l'histoire littéraire (Delfau \& Roche, 1977 ; Goulemot, 1979 ; Béhar \& Fayolle, 1990 ; Moisan, 1987 ; etc.) selon plusieurs voies de développement dont G. Idt (1977) précise les grandes directions. En appui avec l'histoire culturelle (P.Ariès), l'histoire des mentalités (M. Vovelle), il s'agira d'inscrire le texte dans son contexte de production, sous un angle 
synchronique et hétérogène (rapport entre le texte et les autres discours tenus à son époque) ou, d'un point de vue homogène et historique, en élaborant une histoire de la littérature qui porte sur les œuvres et l'évolution de leurs composantes (genres, formes, motifs, techniques, personnage, etc.). À quoi on peut ajouter l'apport de la sociocritique (C. Duchet, P.-V. Zima) et de ses multiples variantes qui, comme le rappelle P. Popovic (2011) dans un article de synthèse, se veut une lecture sociale des œuvres. Quant à la sociologie de la littérature, elle s'intéresse aux conditions sociales et institutionnelles de production, de diffusion et de consommation des œuvres, qu'elle soit le fait d'historiens (R. Darnton, D. Roche) ou de sociologues (R. Escarpit, R. Ponton, C. Charle).

Sur le plan didactique, il est certes utile de fournir aux élèves des tableaux chronologiques qui leur permettent d'avoir un cadre historique. Cela fait partie du travail sur les « fiches de lecture » (Bessonnat, 1996 ; Guernier, 2006) à condition qu'elle ne soit pas technicisée et uniformisée (Privat \& Vinson, 1996). Cette conscience historique et culturelle sera d'autant plus assurée qu'elle fait l'objet d'un travail interdisciplinaire avec les professeurs d'histoire, (voir, outre Le Français aujourd'hui $\mathrm{n}^{\circ} 49$ ou Pratiques $\mathrm{n}^{\circ}$ 69, les articles de J.-F. Inisan, O. Chardon et F. Roelens, 2007 ; V. Bonnefille et A. Noyère, 2007), d'arts plastiques, de musique ou de sciences économiques et sociales. Il importe surtout, comme le montrent les numéros 72 et 73 du Français aujourd'hui, de fournir aux élèves un minimum de conscience historique. Pour ce faire, il importe d'organiser des apprentissages qui se situent à des niveaux différents :

- donner aux élèves, ou leur faire chercher, les sources (paratextes, métatextes, discours critiques, correspondances d'auteurs...) qui éclaireront les conditions de la production du texte ;

- mettre les élèves dans des situations dites de résolution de problème, c'est-à-dire leur proposer des tâches caractérisées par un obstacle que l'élève ne peut surmonter sans acquérir des connaissances nouvelles (à titre d'exemples : au niveau du collège, justifier dans un petit corpus de descriptions celles qui sont à fonction mathésique ou sémiosique et réécrire un extrait descriptif en changeant de point de vue - Petitjean, 1987; au niveau du lycée, travailler sur les stéréotypes thématiques et idéologiques à partir de L'Écume des jours de B. Vian - E. Falardeau, 2003 ; réfléchir à l'aide d'un tri de textes aux indices qui permettent de situer un texte dans son contexte de production - C. Biguet, M. Mougenot, 1985 ; etc.).

Faire de l'histoire littéraire autrement, c'est aussi bénéficier des apports d'une histoire culturelle qui s'intéresse aux "modèles symboliques et aux formations imaginaires caractéristiques d'une culture donnée » (Chaperon \& Reichler, 2000). C'est ainsi, comme le suggère B. Vouilloux $(2004: 250)$ qu'une

« comédie de Molière peut être lue du strict point de vue de l'histoire des mœurs, abstraction faite de ses propriétés littéraires; elle sera alors mise sur le même plan que d'autres sources d'information, telles que la littérature de colportage, les almanachs, les pièces d'archives, etc. : l'histoire des "mentalités" (Philippe Ariès, Alain Corbin...) ne procède pas autrement ».

L'intérêt de ce type d'usage des textes littéraires, dans la mesure où les œuvres réfèrent à des représentations collectives de notre société, des modes de sentir d'une époque je songe, par exemple à «L'histoire des larmes » d'A. Vincent-Buffault (1986) -, est de mobiliser la curiosité des élèves tout en leur procurant des repères historiques. Ces derniers concernent des attitudes ou des moments culturels significatifs (artistiques, scientifiques, éducatifs, etc.) susceptibles de construire des valeurs communes tout en donnant aux élèves le sens du relatif nécessaire à leur compréhension des autres. À 
condition, toutefois, comme le souligne Y. Séité (2000) que l'historien ne dénie pas les propriétés littéraires et fictionnelles de ces textes et «croit pouvoir se dispenser de cette confrontation minimale aux textes qui a nom de lecture ». Le mode didactique de transposition de ces savoirs qui a été privilégié par l'institution est l'activité dite du "groupement de textes" (voir le numéro 97 du Français aujourd'hui) ou le plus récent dispositif de la "séquence» tel qu'il peut porter sur les problématiques du type l'« altérité » ou de l'« éducation » préconisées par les IO du lycée en vigueur. J’ai bien dit "problématique " et non pas "thématique ", dont Y. Reuter (1982) a analysé les errements passés. Comme l'écrit C. Reichler (1992: 43) :

«Tout est organisé autour d'un problème qu'on ne perd jamais de vue, et qui joue tantôt le rôle incitatif d'énigme qu'il s'agit de déchiffrer, tantôt celui d'instrument dans la construction d'une cohérence : d'un instrument tiré des œuvres mêmes et de leur regroupement orienté. Les textes fonctionnent effectivement comme des interprétations l'un de l'autre, et en même temps, ils recontextualisent les éléments repris, les réemploient dans des ensembles qui les modifient: par là deviennent perceptibles les modalités socio-culturelles qui infléchissent les significations et les insèrent dans des situations historiques nouvelles ».

Encouragées par l'Institution et par les textes officiels du collège et des lycées (voir infra l'«Organisation de l'enseignement de l'histoire des arts») mais loin d'être généralisées dans les pratiques, certaines formes de collaboration sont possibles entre les disciplines français (surtout littérature) et histoire. C'est ainsi que M. Laparra (1991) s'intéresse aux manières d'écrire l'histoire sous une forme vulgarisée et aux difficultés que rencontrent les élèves dans la lecture de ces textes. F. Audigier et C. Ronveau (2007), de leur côté, montrent à propos de La Grande Guerre (1914-1918) que si les modes d'entrée dans la question du récit sont différents (dans un cas, l'accent est plutôt porté sur la reconstruction du référent et l'exigence de vérité, dans l'autre cas, il est plutôt mis sur les procédés de construction du monde de la fiction), le dialogue peut s'avérer fructueux pour chacune des deux disciplines. Plus généralement, comme l'atteste le numéro 134-135 de Pratiques, le « récit » n'est pas qu'un type ou un genre de textes mais un mode d'intellection du réel et, ce faisant, un objet disciplinaire transdisciplinaire par excellence.

Il revient, de ce fait, à l'intervention enseignante de ne pas enfermer les élèves dans la seule « connaissance sensible» des textes mais, je le redis, de développer leur capacité interprétative par l'apport de savoirs latéraux (linguistiques, génériques, intertextuels, culturels et historiques...).

En ce sens, les nombreux travaux portant sur les pratiques de la classe ont, comme on l'a vu, le mérite de chercher des réponses à un certain nombre de questions :

- comment prendre en compte « le sujet lecteur " au sein de la classe, ses représentations par rapport à la lecture comme à l'écriture littéraire ainsi qu'aux exercices scolaires ?

- comment, dans l'espace de la classe, concilier les lectures singulières des élèves et la lecture collective d'une « communauté interprétative » telle qu'elle relève de la doxa scolaire?

- comment développer la lecture littéraire alternant des postures de participation (présentation de lectures « coup de cœur »; journal de lecture; débat « littéraire », etc.) et de distanciation (lectures analytiques, commentaires, etc.) ?

- comment développer et favoriser une pratique effective de la littérature qui implique une diversité d'activités d'écriture (réflexive ou créative) et d'échanges oraux (explications, débats, comptes rendus, etc.) ?

- comment faire dialoguer les disciplines dans le but d'élaborer une culture commune? 


\section{4. État actuel des théories de référence et orientations des recherches en didactique de la littérature}

\subsection{Du texte au discours}

«Où en est la théorie littéraire?» Tel était le titre, en 2000, du numéro 37 de la revue Textuel. Je reformulerai la question en me demandant, de façon plus restreinte, non pas où en sont les théories de la littérature dont on sait qu'elles sont historiques, anthropologiques, sociologiques, herméneutiques, philosophiques, poétiques, génétiques etc., mais que reste-t-il du paradigme formaliste et structuraliste des années 60-70 et des travaux de narratologie des années 70-80? À la lecture des revues de théorie littéraire - je me limite au domaine français - on constatera le retrait des uns (Todorov s'est tourné vers l'éthique et Genette vers l'esthétique), le scepticisme d'autres (Compagnon, 2000) quand ce n'est pas la régression polémique de certains (voir la charge critique de C. Bremond et T. Pavel, 1999, contre le S/Z de R. Barthes, qui n'a rien à envier à l'ancien pamphlet de R. Picard). Plus généralement, on peut dire que la théorie littéraire, comme le diagnostique A. Viala (2000), est entrée dans les usages universitaires et se diffuse par le truchement de recueils de textes théoriques (voir, par exemple, les ouvrages publiés dans la collection «GF-Corpus » de Flammarion et dont les titres sont évocateurs: Le genre littéraire, La fiction, L'intertextualité, Le roman, Le personnage, etc.). Plus encore, elle a été transposée dans le champ scolaire, au grand dam d'A. Compagnon (2000a) qui n'y voit «qu'une petite technique pédagogique souvent aussi desséchante que l'explication de texte à laquelle elle s'en prenait alors avec verve ». Finis les débats, voire les polémiques, du passé. On a l'impression que la boite à outils fonctionne, à travers les études d'un genre, d'une forme, d'un procédé ou d'une œuvre. On assiste à une poursuite toujours possible des "nouvelles critiques " mais sans renouvellement majeur des théories, à la différence de ce qui se passe dans les sciences du langage dont on ne peut que regretter leur séparation institutionnelle avec les études littéraires. À ce propos, analysant les « liaisons tumultueuses » entre linguistique et littérature, M.-A. Paveau (2011) nous rappelle que "la littérature est faite avec de la langue». Ce faisant, elle propose un panorama des ressources disponibles qui permettent de problématiser la «question de l'analyse linguistique des textes littéraires en contexte didactique ». On ne peut que regretter que les partisans des humanités ignorent à ce point combien les sciences du langage, demeurent un adjuvant incontournable pour la description des textes littéraires (voir Jaubert, 1990 ; Maingueneau, 2003) et les nombreux articles de la revue L'Information grammaticale. À titre d'exemple, on sait que l'un des reproches que l'on fait aux écrits d'invention des lycéens est qu'ils sont saturés d'expressions toutes faites ou idiomatiques (voir Pratiques, $\mathrm{n}^{\circ}$ 159-160) et il n'est pas inutile de leur montrer combien il est possible de prendre de la distance avec ces expressions en les détournant, comme le montrent L. Perrin (2013) et S. Milcent-Lawson (2013), à propos de Giono et de Beckett. Je pense aussi à ces études linguistiques qui s'intéressent à la manière dont les émotions sont rendues manifestes dans les textes (C. Plantin, C. Kerbrat-Orrechioni, A. Rabatel...) ainsi que ceux qui portent sur ces deux registres que sont l'humour et l'ironie (J.Bres, P. Charaudeau, A. Rabatel, etc.). Ils appartiennent à des paradigmes aussi divers que l'analyse de discours (Maingueneau, 2004 ; ainsi que Amossy \& Maingueneau, 2003) ou les théories du style (Molinier \& Cahné, 1994 ; Gouvard, 2005 ; Petitjean \& Rabatel, 2007) et plus 
précisément de l'idiolecte (Détrie, 2005 ; Neveu, 2005 ; Philippe, 2005 ; Rabatel, 2007 ; Vaudrey-Luigi, 2011). Comme je l'ai montré par ailleurs (Petitjean, 2009, 2010), les notions de genre et de style, qui sont autant de concepts intégrateurs chargés d'une longue histoire depuis la rhétorique et la poétique antiques, ont fait l'objet depuis une dizaine d'années de descriptions linguistiques susceptibles de renouveler leur traitement didactique (voir Pratiques, $\mathrm{n}^{\circ} 135-136,157-158$ ). Il en va de même, pour prendre un autre exemple, des notions de polyphonie et de dialogisme (Pratiques, $\mathrm{n}^{\circ}$ 123-124). L'intérêt heuristique de ces notions est de mieux rendre compte du fait que les textes littéraires mettent en scène des voix et des points de vue divers (voir Rabatel, 1998, 2005, 2008). Il apparait ainsi qu'en liaison avec le travail en langue, il s'avère utile pour la compréhension des textes par les élèves d'attirer leur attention sur les caractéristiques linguistiques des attitudes élocutives, les manifestations des points de vue énonciatifs ou les traces de la subjectivité énonciative (voir, par exemple, Rabatel, 2005 ; Auricchio, Masseron \& Perrin-Schirmer, 2004 ; Leclaire-Halté, 2004 ; Étienne, 2011). L'autre intérêt d'une conception dialogique des œuvres est de prendre en compte, en synchronie comme en diachronie, leur rapport à la langue et leur ouverture à d'autres textes. Au niveau de la langue, on souscrit à ce que propose D. Maingueneau (2004: 140), à savoir que :

«L'écrivain n'est pas confronté à la langue, mais en interaction de langues et d'usages, à ce que l'on appellera une interlangue. Par là, on entendra les relations, dans une conjoncture donnée, entre les variétés d'une même langue, mais aussi entre cette langue et les autres, passées ou contemporaines. C'est en jouant de cette hétéroglossie foncière, de cette forme de "dialogisme" (M. Bakhtine) que peut s'instaurer une œuvre. [...] Cette gestion de l'interlangue, on peut l'envisager sous sa face de plurilinguisme externe, c'est-à-dire dans leur relation des œuvres aux autres langues, ou sous sa face de plurilinguisme interne, dans leur relation à la diversité d'une même langue. "

Qu'une même œuvre puisse faire entendre des fragments de langues diverses, nombre de travaux en apportent la preuve. Pour ne prendre que deux exemples relevant des travaux collectifs dont j'ai eu la responsabilité, je me réfère à l'analyse de R. Michel (2004) de «l'entre-deux-langues» des romans d'Assia Djebar et à l'étude d'A. Chatton (2011) de l'usage des langues étrangères dans l'œuvre théâtrale de B.-M. Koltès. Quant à la pluriglossie interne, je renvoie aux procédés stylistiques employés par les romanciers ou par les dramaturges pour mettre en fiction et en diction les voix populaires (voir Petitjean \& Privat, 2007 ; Petitjean \& Favart, 2012 ; Philippe, 2009 ; etc.). Il ressort de ces études qu'il existe une « langue littéraire » dont G. Philippe et J. Piat (2009) contribuent à relater l'histoire en reconstituant les «faisceaux de traits langagiers" ou les "patrons discursifs» (l'usage de l'imparfait des romanciers naturalistes; la représentation du discours intérieur dans le roman de l'entre-deux-guerres; etc.) qui permettent de mettre en relation les usages communs de la langue et les pratiques singulières des styles d'auteurs. Pour décrire l'inscription d'un texte ou d'un genre dans une situation discursive, on se référera aussi à la notion de "classe généalogique " (Schaeffer, 1999), de "lignée " générique (Rastier, 1999) ou de "mémoire discursive » (Paveau, 2013b). Il s'agit de montrer combien les genres et les textes littéraires sont pris dans des jeux intertextuels/interdiscursifs (voir Paveau, 2010), hypertextuels, voire dans des relations d'inter-articité. Pour l'écriture dramatique, je renvoie à A. Petitjean (2003). Ils impliquent des sources et des reprises, une tradition et une mémoire, et expliquent, outre les phénomènes de variation et d'innovation générique, le fait que, contrairement aux sciences où le passé renvoie à des durées brèves sous la pression de 
la falsification des démonstrations antérieures, pour la littérature le passé est toujours présent, que les créations nouvelles s'en inspirent ou s'en détachent. Il s'ensuit, si l'on veut opérer la «mise en perspective des œuvres littéraires" dont parlent les programmes de 2008, qu'il importe de procurer aux élèves les savoirs historiques et contextuels, formels et esthétiques dont ils ont besoin (références culturelles et allusions intertextuelles) pour comprendre et interpréter les textes. Qu'on me permette de puiser dans mon propre fonds d'articles, mais je pense avoir montré, pour ne prendre qu'un exemple, la polyphonie énonciative et l'intertextualité du Candide de Voltaire (voir Petitjean, 1988) ainsi que ses possibles réécritures transgénériques (Dufiet \& Petitjean, 2013). Pour ce faire, on aidera les élèves à constituer des dossiers documentaires (avoir, par exemple, pour étudier un roman de Zola, un minimum de connaissances déclaratives concernant l'auteur, le réalisme/naturalisme, le contexte historique et Napoléon III). C'est à ce niveau que peut se défendre le principe de la " lecture en réseau ", comme le montre A.-M. Tauveron (2000) à propos d'une étude de L'œuvre de Zola ou M.-C. Kirpalani (2000) pour le genre de la nouvelle.

Passer des textes aux discours, c'est aussi prendre en compte l'apport de la critique génétique dont les travaux, qu'ils soient réalisés par des littéraires (P. M. de Biasi, J. Bellemin-Noël, R. Debray-Genette, J. Neefs...) ou par des linguistes (J.-L. Lebrave, A. Grésillon, I. Feneglio, S. Pétillon...) ont pour objet de rendre compte des étapes successives de la genèse d'une œuvre et des mécanismes qui sous-tendent sa production. On ne compte plus aujourd'hui, outre la revue Genesis, les numéros de revues, les livres ou les articles qui portent sur l'étude des manuscrits. Ils permettent de rendre compte de l'écriture « en mouvement» (Herschberg-Pierrot, 2005) au sens où il s'agit de constituer l'archive génétique et son contenu (notes, plans...), de décrire les matérialités du brouillon (choix des instruments d'écriture, modes d'organisation de la page - linéaire ou tabulaire), mélange ou non de verbal et d'iconique, de reconstruire les opérations scripturales (ajouts, effacements, déplacements, substitutions, reformulations, commentaires métadiscursifs), de suivre les moments et le rythme de la réécriture (variante immédiate ou après relecture différée), etc. L'intérêt de ces recherches est de montrer que les manuscrits sont à la fois des analyseurs contextuels (positionnement de l'auteur au sein d'un mouvement ou d'une esthétique, anticipation de la censure...), des révélateurs du style d'un auteur et surtout d'attester de l'existence d'une "intentionnalité processuelle» (Schaeffer, 1992) qui est celle de l'auteur. On trouvera dans Pratiques des articles théoriques de type génétique (Grésillon, 1988, 1994 ; Scarpa, 2000; Herschberg-Pierrot, 2007; Bikialo \& Pétillon, 2007) et d'autres qui confirment leur intérêt didactique pour l'acquisition de savoirs littéraires et de construction de compétences scripturales (voir le numéro 105-106 de Pratiques intitulé «La réécriture » ou Legoff, 2005). À ce propos, on appréciera que les programmes de 2008 , réaffirment, après ceux de 2000, l'intérêt de faire découvrir par les élèves la «fabrique des textes » et, de ce fait, de leur faire "prendre conscience du caractère relatif des notions d'originalité et de singularité stylistique ». On regrettera, par contre, que cet objet d'étude soit réservé aux premières $L$ alors qu'il serait plus judicieux, sauf à fétichiser les manuscrits d'écrivains, de faire un lien avec les processus rédactionnels des élèves (voir Pratiques, ${ }^{\circ} 49$; Boré, 2000, 2004 ; Plane, 2000).

Quant à la singularité stylistique, elle s'objective aussi par la pratique de l'« écriture mimétique", en particulier le pastiche et la parodie (Aron, 2004; Sangsue, 2007). Comme l'attestent les numéros 42 de Pratiques («L'écriture imitation») et 5 de la revue 
Formules («Pastiches, collages et autres réécritures») et, plus récemment, les volumes 60 et 61 de Modèles linguistiques, consacrés au pastiche, ces activités scripturales permettent de faire de l'histoire littéraire pour au moins deux raisons. Elles sont l'occasion de faire découvrir aux élèves que le pastiche (il en va de même pour la parodie) est une pratique familière aux écrivains dans leur phase d'émergence ou relève d'une forme de la critique (Reboux et Muller, Guenel, Rambaud...) et appartient de ce fait au jeu institutionnel de la littérature (voir Genette, 1982 ; Petitjean, 1984; Bilous, 2003, 2004; Bilous \& Bilous, 1988). Elles sont surtout enrichissantes pour la connaissance du style d'un auteur, un moyen, à la fois efficient et ludique, de développer les compétences en lecture comme en écriture des élèves et l'occasion de vérifier s'ils se sont approprié les savoirs historiques et textuels nécessaires à la réussite du pastiche (voir Aron, 1984b ; Bessonnat \& Schnedecker, 1990).

Pour compléter ce panorama des théories susceptibles d'être transposées, j'évoquerai les théories de la fiction (Searle, Genette, Ricoeur, Schaeffer...) à partir desquelles se pensent l'opposition entre récit factuel et récit fictionnel ainsi que les différents contrats de lecture correspondants aux genres littéraires (en particulier le roman) les mieux représentés dans l'enseignement du français.

132 La solution didactique la plus simple est de conforter l'assimilation par les élèves du domaine de la fiction à celui de l'imaginaire et de l'invention. L'inconvénient d'une telle représentation est de confondre le discours fictionnel avec son référent et surtout de faire dépendre la «fictionnalité » du discours des connaissances encyclopédiques du lecteur. Il est plus judicieux de leur montrer que la fiction est une «modélisation analogique " de la réalité et qu'elle s'incarne dans des supports symboliques aussi différents que le récit verbal, les pièces de théâtre, les histoires drôles, le cinéma, les bandes dessinées, la peinture, la photographie... Ils ont en commun une même intentionnalité («la feintise ludique partagée ») et requièrent une attentionnalité spécifique. Le problème de l'objectivation du discours fictionnel étant complexe (voir Bernard, 2000), il serait trop long de mener un examen des ces différentes conceptions, qu'elles soient ontologique (Schaeffer, 1999), textuelle (Hamburger, 1977), sémiotique (Pavel, 1988), pragmatique (Searle, 1982 ; Genette, 1991), énonciative (Philippe, 2000). Je retiens pour justifier l'intérêt didactique des fictions, en premier lieu, qu'elles réfèrent indéniablement à «nos principaux soucis sociaux ou existentiels» (Pavel, 1998 186): "La naissance, l'amour, la mort, le succès et l'échec, le pouvoir et sa perte, les révolutions et les guerres, la production et la distribution des biens, le statut social et la moralité, le sacré et le profane, les thèmes comiques de l'inadaptation et de l'isolement, les fantaisies compensatrices, etc., traversent toute l'histoire de la fiction, depuis les mythes les plus anciens jusqu'à la littérature contemporaine. »

133 Point de vue similaire que celui de V. Jouve (2010:149) qui écrit: "L'intérêt d'une lecture tient, en effet, soit à la découverte d'une dimension de nous-mêmes jusque-là inexplorée, soit au sentiment d'être confronté à une interrogation fondamentale. [...] Certaines composantes de l'existence - le désir, la souffrance, la conscience de la mort, etc. - intéressent, par la force des choses, chacun d'entre nous. »

134 L'autre intérêt des fictions et plus particulièrement des récits fictionnels est qu'elles ont des fonctions cognitives importantes, comme le rappelle J.-M. Schaeffer (1999: 130): «La simulation ludique possède au moins trois fonctions: elle permet d'apprendre un comportement sans être soumis à la sanction immédiate de la réalité ; 
elle nous apprend à modéliser des situations susceptibles de se présenter à l'avenir ; et enfin, elle nous permet de nous habituer peu à peu à des situations dysphoriques que nous devons affronter dans la vie réelle ».

Dit autrement, l'intérêt de la machine fictionnelle par rapport à l'économie psychique du lecteur réside, comme le souligne Y. Citton (2007), dans le fait que, "d'une part, elle déplace notre expérience affective d'un "contexte réel" vers un "contexte fictionnel" (délocalisation paratopique); que d'autre part, grâce à ce déplacement et aux affects de latéralisation qu'il autorise, elle réorganise nos affects en frayant de nouvelles voies de modes et de liaisons entre nos peurs, nos espoirs, nos envies et nos dégoûts ».

Si de telles opérations cognitivo-affectives sont possibles (identifier le général derrière le particulier) cela tient à la "typicité » et au caractère emblématique, exemplifiant (Goodman, 1990) et intensifiant (Elgin, 1992) des œuvres. Ce que confirme V. Jouve (2010: 156) : "C'est la typicité qui me permet de considérer Madame Bovary comme l'expression d'un mal-être métaphysique et Les Misérables comme représentatifs des dégâts humains consécutifs à l'injustice sociale. Les œuvres les plus marquantes seraient donc exemplaires de caractéristiques fondamentales de notre condition (et pas seulement de traits sociaux et psychologiques relatifs à un contexte culturel particulier ».

137 Je retiens, aussi, concernant leur réception, que les fictions induisent, génèrent, autorisent, de la part du lecteur, un comportement cognitif «bi-planaire " (Lotman, 1973), un «état scindé » (Schaeffer, 1996), en tension entre, d'un côté, une immersion dans la fiction (empathie, identification, reconnaissance, etc.) et, de l'autre, une neutralisation consciente de ses effets, voire une possible dissociation mentale sur la base d'une convocation de connaissances latérales (historiques, contextuelles, intertextuelles). L'ensemble se réalise sous des formes diverses, depuis des moments métalecturaux, au cours de la lecture, jusqu'à la production de discours métatextuels. Dans tous les cas, l'effort de "faire semblance " (Pavel, 1988) que doit accomplir le lecteur est proportionnel au niveau de « bienveillance » du texte (degré de perméabilité référentielle et de complétude informationnelle, densité de facteurs antimimétiques ou anticonventionnels, type d'élaboration et de condensation stylistique). À propos de la métatextualité, on pourra regretter que les programmes officiels ne donnent pas la possibilité aux élèves, en particulier au niveau des lycées, de leur faire découvrir les variétés du discours critique selon ses agents (de l'écrivain au professionnel), ses formes textuelles (paratextes - notes, variantes, glossaires, index - ou péritextes préfaces, postfaces, prières d'insérer...), ses genres attestés : dissertatifs (examens, discours, observations...), dialogués (entretiens - Chapelain), dialogues théâtraux critiques (Molière, Ionesco), polémiques (manifestes, pamphlets, lettres ouvertes), ludiques (pastiches de Pérec ou d'Eco), fictifs (Borges).

Faut-il rappeler à ceux qui, comme M. Jarrety (2000), pensent qu'il faudrait limiter les exercices du second cycle à la dissertation et au commentaire que les textes officiels, jusque dans les années 50, préconisaient "diverses formes de lecture ", qu'elles aient lieu en classe (lectures courantes, suivies, dirigées, expliquées), sous la forme d'extraits ou d'œuvres complètes, ou qu'elles se fassent hors classe («lectures complémentaires ») et pouvant faire l'objet d'un résumé, d'un compte rendu ou d'un exposé ? Faut-il rappeler aussi que selon ces activités ou en fonction des différentes postures commentatives (Daunay, 1997; Delcambre, 1989), les œuvres littéraires se 
prêtent à différents types de jugements qui vont de la réaction émotionnelle et de l'appréciation subjective à l'analyse distanciée et argumentée (voir infra) ?

\subsection{Orientations des recherches en didactique de la littérature et perspectives}

139 Pour poursuivre avec l'actualité des recherches en didactique de la littérature, je mentionnerai des orientations de recherche et des mesures institutionnelles qui méritent examen dans la mesure où elles « impactent », pour reprendre un technolecte en usage chez les décideurs, l'enseignement de la « lecture littéraire».

Au niveau des recherches, j'évoquerai ce qu'il est convenu d'appeler les "gestes professionnels » (Jorro \& Croccé-Spinelli, 2010) qui préparent, accompagnent et sanctionnent l'approche des textes. Cela implique de porter le regard de l'observateur sur les activités réelles des élèves et sur le travail des enseignants. Certes, s'intéresser aux pratiques d'enseignement à l'aide d'observations de classes, d'enquêtes (voir supra) et d'entretiens n'est pas nouveau (voir Manesse \& Grellet, 1994, pour le collège ; Veck, 1988, 1990, 1994, pour l'enseignement de la littérature dans les classes de lycée). Il s'agit de décrire les pratiques et d'en définir les constituants (rapports de l'enseignant aux textes officiels et aux prescriptions des programmes, modes d'utilisation des manuels, gestes scolaires qui préparent et accompagnent l'acte pédagogique). C'est ainsi que M.F. Chanfrault (2001a) observe que la pratique du surlignement tend à figer la lecture méthodique dans des relevés de marques formelles. Dans une autre étude, M.F. Chanfrault-Duchet (2001b) montre combien les enseignants se sentent en insécurité en matière d'interprétation littéraire suite à la mise en application des programmes de 2000 et des nouvelles épreuves du baccalauréat. Le constat est assez similaire pour les enseignants de cycle 3 confrontés à la réforme de 2002 en matière de littérature (Louichon, 2008). Il est vrai, cependant, que le spectre des recherches en la matière s'est étendu, comme l'attestent de récentes publications (Bucheton \& Dezutter, 2009; Chabanne \& Dezutter, 2011; Marlair \& Dufays, 2011, Jaubert \& Rebière, 2011). Il s'agit essentiellement de mener des études qualitatives du type de celles réalisées par H. Crocé-Spinelli (2007) qui rendent compte de la complexité des interactions qui se jouent entre les trois pôles que sont l'élève, l'enseignant et l'objet enseigné. Si l'on excepte l'ouvrage dirigé par J.-F. Halté (1990) et quelques articles (Sprenger-Charolles, 1983 ; Cicurel, 2011), l'étude des interactions au cours des situations scolaires d'analyses des textes littéraires n'a pas été privilégiées dans Pratiques. D'où l'intérêt des études qui se focalisent sur ce qu'A.Jorro (2002) appelle la préoccupation d'« ethos" et D. Bucheton (2008) d' " atmosphère ", à savoir ces interventions aux cours desquelles l'enseignant prépare la rencontre des élèves avec les œuvres. Comme le montrent J.L. Dumortier (2006) et J.-L. Dumortier et alii (2011), ces discours d'escorte, qu'ils appellent les "discours incitatifs», tels qu'ils portent sur un auteur, un texte ou un extrait, ont pour finalité principale de donner aux élèves l'envie de lire un texte. Que ces gestes professionnels et les représentations concernant la littérature et son enseignement soient pour une large part dépendants de la formation initiale des enseignants, différentes études l'attestent. Je pense à A. R. De Beaudrap $(2004,2005)$ qui s'intéresse aux «représentations professionnelles » des futurs professeurs de lycée et collèges de l'IUFM des Pays de la Loire, à J. Emery-Bruneau (2011) enquêtant sur les étudiants québécois en formation à l'enseignement du français au secondaire ou à E. Falardeau et D. Simard (2011) qui observent comment deux enseignantes prennent 
en compte le rapport à la culture des élèves. Selon les cas, il apparait que ces représentations divergent. Les uns privilégient les connaissances littéraires et culturelles ainsi que la réflexion et la distanciation. Les autres se représentent plutôt comme des "animateurs culturels" accordant une attention quasi exclusive aux aspects psycho-affectifs et subjectifs de la lecture des textes littéraires. Il serait nécessaire, pour prendre la mesure des pratiques actuelles des jeunes collègues sortant des masters en France de mener des enquêtes similaires telles qu'ont pu les faire A. Bounoure, M. Delclaux et J. Pastiaux (1987).

Quant aux mesures institutionnelles, je fais allusion au fait que depuis quelques années l'institution scolaire française affirme sa volonté d'instituer des «enseignements artistiques et culturels » sur la base d'activités trans- ou pluri-disciplinaires impliquant la participation d'intervenants extérieurs. Je pense aux PAE (projets d'action éducatifs) ou au TPE (travaux personnels encadrés) et, plus récemment, au décret du 11 juillet 2006 sur le «Socle commun de connaissances et de compétences». Parmi les sept connaissances mentionnées, la discipline "français » est plus spécialement concernée par deux d'entre elles : « la maitrise de la langue française » et « la culture humaniste ». La première " passe par la capacité à lire et comprendre des textes variés, la qualité de l'expression écrite, la maitrise de l'expression orale, l'apprentissage de l'orthographe et de la grammaire, l'enrichissement quotidien du vocabulaire ", confirmant ainsi le rôle $\mathrm{du}$ «français » comme «méta-discipline» pour l'acquisition de «compétences littéraciques ». La seconde, qui nécessite l'interaction d'autres disciplines, « contribue à la formation du jugement, du goût et de la sensibilité et permet d'acquérir des repères : en histoire (événements fondateurs), en géographie (paysages et territoires, populations, etc.), en littérature et en arts (les grandes œuvres). Une approche sensible des œuvres initie l'élève à l'histoire des arts. Il est engagé dans des pratiques artistiques personnelles".

142 La préconisation d'une mise en synergie entre champs disciplinaires va connaitre un seuil supplémentaire avec le décret du 28 août 2008 sur l'«Organisation de l'enseignement de l'Histoire des Arts ». Je cite :

« Aux trois niveaux du cursus scolaire, École primaire, Collège, Lycée, l'histoire des arts instaure des situations pédagogiques pluridisciplinaires. Son enseignement implique la constitution d'équipes de professeurs réunis pour une rencontre, sensible et réfléchie avec les œuvres d'art de tout pays et de toute époque. Il est aussi l'occasion de renforcer, autour d'un projet national conjoint, le partenariat entre les milieux éducatifs et les milieux artistiques et culturels.»

On ne se privera pas d'apprécier que l'institution, même si ce n'est pas son premier essai (voir le rapport Bourdieu-Gros de 1989 ou les textes Lang-Tasca des années 2000), finisse par reconnaitre la validité de ce qui a été la pratique d'enseignants novateurs comme en témoigne le fait que Pratiques a de longue date réfléchi et fait des propositions qui vont dans le sens de certains attendus du texte officiel :

- instaurer des «situations pédagogiques pluridisciplinaires et partenariales» (voir le $\mathrm{n}^{\circ} 31$, « Travailler en équipe », $\mathrm{n}^{\circ} 36$ « Travailler en projet », n 15-16 sur le « théâtre », etc.;

- favoriser le «dialogue entre les disciplines» (voir en particulier, pour le rapport texte/ histoire les numéros 69 et 133-134);

- «favoriser la créativité de l'élève » (voir les numéros 61, consacré aux ateliers d'écriture, et 127-128, à l'écriture d'invention) ; 
- «aiguiser les capacités d'analyse de l'œuvre d'art » (voir les nombreux numéros consacrés au commentaire, à l'interprétation des textes mais aussi à l'analyse de l'image $\left(\mathrm{n}^{\circ} 7-8\right.$, $\left.\mathrm{n}^{\circ} 18-19\right)$

Par ailleurs, pour avoir été responsable de la Culture à l'université Paul Verlaine-Metz et avoir impulsé, dans ce cadre, tant la politique des «ateliers de pratiques culturelles ", les activités du théâtre Bernard-Marie Koltès de l'université, reconnu "scène conventionnée » par le ministère de la Culture, que le partenariat avec le centre Pompidou-Metz, je me sens fortement concerné par cette nouvelle directive. Ce qui ne m'empêche pas de rester dubitatif quant aux conditions de sa mise en œuvre compte tenu de la formation monodisciplinaire des enseignants de collèges et de lycées et de l'aspect encyclopédique du projet. En effet, alors que le volume horaire qui lui est alloué de la seconde à la terminale est de 24 heures, les objets d'études concernent « six grands domaines artistiques : « arts de l'espace, arts du langage, arts du quotidien, arts $\mathrm{du}$ son, arts du spectacle vivant arts du visuel ». Ils sont à problématiser selon quatre «champs» (anthropologique, historique et social, technique, esthétique) et selon un empan historique qui va de l'Antiquité au $\mathrm{xx}^{\mathrm{e}}$ siècle au collège et $\mathrm{du} \mathrm{xVI}^{\mathrm{e}}$ au $\mathrm{xIX}^{\mathrm{e}} \mathrm{au}$ lycée, puisque le $\mathrm{xx}^{\mathrm{e}}$ est réservé à la terminale où le «français » n'est plus obligatoire. On présume, face à la «liste de thématiques » imposée, la parcellisation des objets d'études et la superficialité de leur traitement. On imagine aussi l'inconfort et l'insécurité des enseignants (sans formation particulière) mis dans l'obligation d'assumer une matière "transversale » et la perte de repères disciplinaires des élèves, alors qu'ils sont censés être évalués. Comme l'écrit J.-C. Chabanne (2013:55) :

\begin{abstract}
«La commande institutionnelle invite à brouiller les frontières disciplinaires traditionnelles en permettant à des enseignants de matières différentes sinon d'intervenir ensemble, du moins à coordonner leurs projets autour d'objets, de démarches, d'objectifs partagés ou coïncidents. Cette "invitation" à inventer ce que l'on pourrait appeler de l'interdidactique serait à interroger pour ce qu'elle crée d'avancées autant que de difficultés et de désarroi ».
\end{abstract}

145 Je m'interroge aussi à propos de la formulation de certains objectifs alloués à ce nouvel enseignement. Je cite :

«L'enseignement de l'histoire des arts a pour objectif :

- d'offrir à tous les élèves, de tous les âges, des situations de rencontres sensibles et réfléchies, avec des œuvres relevant de différents domaines artistiques, de différentes époques et civilisations ;

- de les amener à se construire une culture personnelle à valeur universelle fondée sur des œuvres de référence ;

- de leur permettre d'accéder progressivement au rang d'«amateurs éclairés", maniant de façon pertinente un premier vocabulaire sensible et technique, maitrisant des repères essentiels dans le temps et l'espace et appréciant le plaisir que procure la rencontre avec l'art ;

- de les aider à franchir spontanément les portes d'un musée, d'une galerie, d'une salle de concert, d'un cinéma d'art et d'essais, d'un théâtre, d'un opéra et de tout autre lieu de conservation, de création du patrimoine artistique ;

- de donner des éléments d'information sur les métiers liés aux domaines des arts et de la culture ».

Le texte officiel présentant comme une évidence l'opposition entre "sensible » et « réfléchi » reproduit, comme l'analyse J.-M. Schaeffer (1996), le préjugé hérité de la philosophie occidentale - il oppose "perception » à « intellection » et "sensibilité » à «intellect »- devenu avec le temps une pensée de sens commun. Il est regrettable de voir cette dernière jouer le rôle d'assise théorique par un texte officiel. Il aurait mieux 
valu distinguer " conduite esthétique ", qui est une activité cognitive tributaire à la fois des modes d'aspectualisation des objets artistiques et du degré d'acculturation de l'interprétant et «jugement esthétique » qui est nécessairement un acte de langage "verdictif » sous la forme d'assertions normatives et subjectives, que cette subjectivité soit individuelle ou collective. Que penser aussi de cette posture lettrée de l'" amateur éclairé » dont parle le texte et à l'ethos duquel on souhaite que l'élève puisse s'identifier, sans que l'on sache à quelle "pratique sociale de référence » (l'artiste, le journaliste, le critique, l'essayiste, l'enseignant, l'amateur, le mondain, etc.) l'assimiler? Si l'on pense qu'une « éducation esthétique » est possible, encore faudraitil faire le distinguo entre "expérience esthétique » et "connaissance des arts " et prendre la mesure de la variété des genres d'écrits sur l'art (Vaugeois, 2005) et de discours critiques, ainsi que de la multiplicité des prédicats esthétiques (Vouilloux, 2005) dont G. Genette (1997) rappelle à juste titre qu'elle est inépuisable (gracieux, fade, puissant, charmant, monotone, etc.). Ce qui pose le problème de l'évaluation de la maitrise par les élèves de tels discours.

Dans ce contexte, sur la base d'un séminaire pluridisciplinaire au sein du Centre de recherche sur les médiations (CREM) de l'université de Lorraine, nous avons initié une réflexion sur plusieurs années en vue d'un numéro de Pratiques en préparation intitulé «Didactiques des médiations de la littérature et des arts » et prévu pour 2016. Dans le prolongement des numéros 43 de Repères (2011) et 182 du Français aujourd'hui (2013), il s'agit de travailler, par l'intermédiaire du séminaire et à l'aide d'un appel à articles à venir, sur les sujets suivants :

- réfléchir au statut d'une discipline d'enseignement (Reuter, 2007a), aux problèmes de pluri/ trans/interdisciplinarité, en recherche, formation et enseignement (Charaudeau, 2010, 2012; Questions de communication, $\mathrm{n}^{\circ} 18$ ), ainsi qu'aux modes de théorisation interdisciplinaires des rapports entre texte et image, visuel et verbalisation (Vouilloux, 1992) et, symétriquement, entre lisible et figurable (Jenny, 1990) ;

- problématiser les notions de médiations et de didactique en lien avec les arts (Chabanne, Parayre \& Villagordo, 2011);

- préciser ce qu'il faut entendre par «capacités d'analyse de l'œuvre d'art » et pour ce faire, examen des concepts de "conduite esthétique ", d'« immersion fictionnelle», de "plaisir désintéressé » et de leur pertinence didactique (Michel) ;

- définir la notion de style à partir d'une approche « pansémiotique » et «transdisciplinaire » (Vouiloux, 1998);

- préciser les différences, d'un point de vue intentionnel et attentionnel, entre texte de fiction et œuvre artistique (Schaeffer) ;

- analyser le texte officiel, ses avancées mais aussi son légitimisme culturel au regard de la sociologie de l'art (Bourdieu, 1992 ; Heinich, 1998);

- examiner les conditions de la mise en œuvre du texte officiel et illustrer par des expériences réalisées dans les classes (école, collège, lycée) rendant compte de situations d'enseignement/apprentissage d'un habitus culturel (Privat);

- cartographier, comme le proposent D. Vaugeois (2005) ou C. Deronne (2011) des genres de discours (spécialisés, journalistiques, ordinaires) sur les œuvres d'art, tels qu'ils « circulent dans différents lieux sociétaux et institutionnels »;

- analyser les différentes modalités de réalisation des textes expographiques (Rigat, 2012), en particulier quand les expositions ciblent un public « jeune »;

- étudier les modalités d'initiation des élèves aux pratiques artistiques à travers les activités de médiation organisées par le musée de Saint-Denis (Ravazzolo); 
- analyser, d'un point de vue de sociologie de la culture les rapports extra-scolaires des élèves aux pratiques artistiques (lectures ordinaires, visites d'un musée, concert, théâtre, vidéo, cinéma...) et les socialités juvéniles qui les manifestent. Commander un bilan relativement actualisé des pratiques culturelles des jeunes Français selon leur appartenance socioculturelle, afin de mesurer comment prendre en compte les usages de la net génération ;

- rechercher un métalangage commun ou différent selon les enseignements littéraires et artistiques en analysant leurs modes de transmission et d'utilisation selon les genres de discours scolaires (parole professorale, travaux d'élèves...) ;

- étudier les gestes professionnels (enseignants, programmes, panneaux des musées...) qui préparent la rencontre avec les œuvres et analyser les modalités d'intervention des partenaires de la culture extérieurs au système éducatif (Lahuerta);

- prendre en compte les objets culturels les plus légitimés mais aussi les séries télévisées, les jeux vidéo...;

- rendre compte de pratiques d'ateliers d'écriture à l'université et des dispositifs culturels susceptibles d'opérer des médiations culturelles (Bisénius-Penin, à paraitre).

\section{Pour ne pas conclure}

L'enseignement du français, compte tenu des difficultés nombreuses que génère, à tous les niveaux du cursus, la scolarisation de masse, implique que l'on ajuste la formation des professeurs aux besoins qui sont les leurs dans l'exercice de leur métier. Ce qui signifie que l'on rompe avec l'idéologie de la grâce culturelle et de la communion lectorale sur laquelle repose la formation du professeur de lettres et que l'on prenne conscience qu'il se doit d'être à la fois un médiateur culturel et un technicien des méthodes. Technicien, dans la mesure où il lui incombe d'initier les élèves aux méthodes de travail qui leur permettent de s'approprier et de restituer les savoirs en français et dans les autres disciplines (Pratiques, $\mathrm{n}^{\circ} 90$ ). Médiateur, au sens où il lui revient de développer le goût de la lecture littéraire et des pratiques artistiques.

Au terme de ce bilan de 40 années de recherches, on peut dire, avec un regard surplombant et rétrospectif, qu'elles se sont diversifiées selon qu'elles sont historiques, descriptives, théoriques, praxéologiques et s'appuient sur des savoirs de référence aussi divers que la sociologie de la littérature, la sociologie des pratiques culturelles et des lecteurs, les théories de la réception, les travaux des historiens du livre, des historiens de l'éducation, ou, encore, sur les sciences du langage, la psychologie cognitive, la génétique et les travaux des littéraires eux-mêmes.

L'une des formes qu'a prise la transposition, et surtout l'élaboration de ces théories, est l'intérêt porté depuis quelques années à la problématique de la " compétence » (Allal, 2002 ; Canvat, 2005), qu'elle soit en lecture (Lafontaine, 2002) ou en écriture (Petitjean, 1998b). Comme en témoignent les nombreux travaux, que j'ai décrits précédemment, il s'agit de compétences complexes, et pour la lecture, un « art de faire » (Certeau, 1980) impliquant diverses dimensions :

- socioculturelle (la compétence présuppose la familiarisation avec les usages de la lecture et de l'intérêt pour la lecture) ;

- psychocognitive (elle implique la maitrise des opérations de traitement des informations);

- sémiolinguistique (elle nécessite des connaissances linguistiques et textuelles); 
- culturelle (elle est facilitée par les connaissances encyclopédiques et culturelles nécessaires au passage de la compréhension à l'interprétation).

\section{dialectiques entre lecture et écriture (on a besoin de lire pour écrire, lire les autres, se lire pour réécrire) et de profiter ainsi d'apprentissages incidents pour la lecture. Précisément :}

- de savoir observer les textes littéraires comme des réservoirs de solutions aux problèmes d'écriture des élèves ;

- de donner aux élèves l'occasion et l'autorisation de produire régulièrement des textes relevant de genres diversifiés et de ne pas réduire leur rapport aux textes par le biais des seuls exercices formels (qu'on les appelle explication de textes, lecture méthodique, lecture analytique) et, pour les écrits, commentaire (Delcambre, 1989) et dissertation (Le Goff, 2009);

- de travailler sur la langue et les discours en fonction des besoins scripturaux des élèves ;

- de leur apporter des connaissances procédurales en matière de lecture et d'écriture.

Psychologues cognitivistes, herméneutes de la réception, sociologues de la lecture, tous ces paradigmes théoriques, malgré leur incompatibilité, convergent pour montrer qu'il existe des mécanismes généraux qui caractérisent l'acte de lecture. Lire est une activité qui présuppose une attention mémorielle sélective, une projection subjective, une « rétention » indispensable mais aussi des phénomènes d'anticipation et de rétroaction que chaque lecteur met en œuvre au cours de ses lectures. Ce qui nécessite la mise en place, en particulier au primaire et au collège, d'exercices de lecture susceptibles de développer les habilités requises pour lire des textes longs ou difficiles. Je fais référence, outre aux activités déjà évoquées, à ce que J.-M. Privat et M.-C. Vinson (2008) ou M.-C. Vinson (2006) appellent les "médiations textuelles» (surligner, annoter, indexer, généraliser, comparer, évaluer, accélérer ou ralentir sa lecture...). Ce sont 
autant d'apprentissages «artisanaux » qui peuvent s'effectuer dès la classe de cours préparatoire (Borowski, 2003).

En attendant de développer les nouvelles perspectives de recherche mentionnées précédemment, je dirai, pour mettre un terme à cette réflexion sur la «lecture littéraire ", que je rejoindrais volontiers la typologie que propose D. Maingueneau (2011), qui distingue :

- la «lecture", activité ordinaire qui «se contente d'accéder à un texte en suivant les contraintes imposées par le genre auquel il est affecté quand on le lit » (journal, recette de cuisine, roman sentimental...) et qui ne préjuge pas des intérêts du lecteur réel ;

- la «lecture hyperprotectrice » qui consiste à lire un livre qui fait "partie d'un Thésaurus partagé par une communauté » et possède de ce fait, qu'il relève de la littérature (Balzac, Flaubert...) ou des sciences humaines, une valorisation certaine ;

- la «superlecture » qui consiste à lire pour rendre compte de sa lecture sous la forme d'un texte oral ou écrit, "conforme à un certain nombre de normes fixées par un cadre institutionnel»;

- l'«hyperlecture » qui vise, comme la précédente, à produire un commentaire «mais à l'intérieur d'un cadre herméneutique ».

"Celui qui propose une stimulante interprétation d'un texte littéraire montre, à la fois qu'il est compétent, qu'il occupe légitimement la place que l'institution lui a reconnue, que le texte est riche de sens, et que la littérature est bien un discours constituant, qui dit des choses essentielles » (ibid. : 55).

Il revient à la didactique de la littérature d'agir de façon à ce que les élèves deviennent de vrais lecteurs, en pratiquant des superlectures, telles qu'elles sont collectives, imposées et guidées, elles-mêmes enrichies par les hyperlectures mais en évitant que l'éclectisme des dernières et la technicité des secondes n'entravent la réalisation des premières.

\section{BIBLIOGRAPHIE}

\section{Ouvrages et articles cités}

ABASTADO, C. (1981) : « La composition française et l'ordre du discours », Pratiques, 29, p. 3-21.

Abdallah-Pretceille, M. (1995) : L'Éducation interculturelle, Paris, Presses universitaires de France (coll. «Que sais-je ?»).

ACHARD-BAYLE, G. (coord.) (2010) : «Linguistique textuelle : états des lieux », Verbum, 2 (XXXII), Presses universitaires de Nancy.

AdAm, J.-M. (1976) : « Langue et texte : imparfait/passé simple », Pratiques, 10, p. 49-68.

- (1977) : « Ordre du texte, ordre du discours », Pratiques, 13, p. 103-111.

- (1984-2011) : Les textes : types et prototypes, $3^{\mathrm{e}}$ éd., Paris, A. Colin (coll. « Cursus »).

- (1985) : Pour lire le poème, Bruxelles, De Boek/Duculot. 
- (1991) : Langue et littérature. Analyses pragmatiques et textuelles, Paris, Hachette.

- (2010) : «L'émergence de la linguistique textuelle en France (1975-2010). Parcours bibliographique en 100 titres », Verbum, 2 (XxxII), p. 237-261.

- (2011) : Genres de récits. Narrativité et généricité des textes, Louvain-la-Neuve, Académia/ Éd. L'Harmattan.

ADAM, J.-M. \& GOLDENSTEIN, J.-P. (1976) : Linguistique et discours littéraire, Paris, Larousse.

ADAM, J.-M. \& LE CLERC-ADAM, B. (1988) : « Un genre du récit : le monologue narratif au théâtre », Pratiques, 59, p. 51-71.

ADAM, J.-M. \& HEIDMANN, U. (2009) : Le texte littéraire. Pour une approche interdisciplinaire, Louvain-laNeuve, Academia/Bruylant.

- (2011) : Textualité et intertextualité des contes, Paris, Classiques Garnier.

AHR, S. (2005) : L'enseignement de la littérature au collège, Paris, Éd. L'Harmattan.

AHR, S. \& Joole, P. (2010) : « Débats et carnets de lecteurs, de l'école au collège », Le Français aujourd'hui, 168, p. 69-82.

Ahr, S., ButLen, M. \& ElAlouf, M.-L. (2012) : « Lectures sur écran, lectures sur papier, discours et représentations des élèves de 15 ans ", Le Français aujourd'hui, 178, p. 66-76.

AlBeRTINI, P. (1987) : « Les mutations de l'enseignement secondaire depuis 1960 », Textuel, 7, p. 68-81.

Allal, L. (1999) : « Acquisitions et évaluations des compétences en situation scolaire », in : J. Dolz \& E. Olagnier (2002), L'énigme de la compétence en éducation, Bruxelles, De Boeck (coll. « Raisons éducatives), p. 77-93.

Amossy, R. \& MAINGUENEAU, D. (dirs) (2003) : L'analyse du discours dans les études littéraires, actes du colloque de Cerisy, sept. 2002, Toulouse, Presses universitaires du Mirail.

AMSTUTZ, C. (1993) : « Des aides à l'écriture narrative (ou comment lancer des élèves en grande difficulté dans l'écriture d'une nouvelle policière ", Pratiques, 78, p. 5-38.

ANToine, G. (1966) : «Stylistique des formes et stylistique des thèmes, ou le stylisticien face à l'ancienne et à la nouvelle critique ", in : G. Poulet (dir.), Les chemins actuels de la critique, Paris, Plon (coll. 10/18), p. 159-168.

ARMAnD, A. (2001) : "Littérature de jeunesse et lecture littéraire », in : Perspectives actuelles de l'enseignement $d u$ français, actes du séminaire national organisé par la direction de l'Enseignement scolaire, ministère de l'Enseignement scolaire, p. 201-210.

Armand, A., Descottes, M., JoRdy, J. \& LANGLADE, G. (1992) : La séquence didactique en français, Toulouse, Bertand-Lacoste (coll. « Didactiques »).

ARON, P. (éd.) (2004) : Du pastiche, de la parodie et de quelques notions connexes, Paris, Éd. Nota bene.

ARON, P. \& Viala, A. (2005) : L'enseignement littéraire, Paris, PUF (coll. « Que sais-je ?»).

ARON, T. (1984a) : Littérature et littérarité : un essai de mise au point, Paris, Éd. Les Belles Lettres (coll. « Annales littéraires de l'université de Besançon »).

- (1984b) : « D'après Flaubert, avec Proust, via Queneau. Pratiques du pastiche à l'université », Pratiques, 42, p. 41-61.

ARRIVÉ, M. (1972) : Les langages de Jarry, Paris, Klincksieck.

- (1973) : « Pour une théorie des textes poly-isotopiques », Langages, 31, p. 53-63.

- (1975) : « Lire, dé-lire », Pratiques, 7-8, p. 7-20. 
AudigieR, F. \& RonveAux, C. (2007) : « Récit et histoire, récit et fiction, Exemple de l'expérience de la guerre ", Pratiques, 133-134, p. 55-75.

Auricchio, A., MAsseron, C. \& Perrin-Schirmer, C. (2004) : « La polyphonie des discours argumentatifs : propositions didactiques », Pratiques, 171-211, p. 171-211.

AYRAUD, M. \& BENOIT, J.-P. (1996) : « La séquence en stage : représentations et pratiques déclarées ", Pratiques, 92, p. 107-123.

BAConNet, M. (2001) : « Points de vue sur l'enseignement du français », in : Perspectives actuelles de l'enseignement du français, actes du séminaire national organisé par la direction de l'Enseignement scolaire, ministère de l'Enseignement scolaire, p. 15-23.

BALIBAR, R. (1974) : Les français fictifs, Paris, Hachette.

BARTHES, R. (1970) : S/Z, Paris, Éd. Le Seuil.

- (1971-2002) : «Réflexions sur un manuel », in : Doubrowsky, S. \& Todorov, T. (éds),

L'enseignement de la littérature,actes du colloque de Cerisy-la-Salle, Paris, Plon, repris dans Euvres Complètes, Paris, Éd. Le Seuil, t. 3, p. 945-951.

- (1972) : Nouveaux essais critiques, Paris, Éd. Le Seuil.

- (1978/2002) : Leçons, in : Euvres complètes, t. V, Paris, Éd. Le Seuil.

- (1975) : « Littérature et enseignement (entretien) », Pratiques, 5, p. 15-21.

- (1984) : Bruissement de la langue, Paris, Éd. Le Seuil.

BARRÉ-DE MINIAC, C. (1995) : « La didactique de l'écriture, nouveaux éclairages pluri-disciplinaires et état de la recherche », Revue française de pédagogie, 113, p. 93-133.

BAudelot, C. \& Establet, J.-C. (1971) : L'École capitaliste en France, Paris, F. Maspero.

- (1992) : Allez les filles, Paris, Éd. Le Seuil.

BAUDELOT, C. \& CARTIER, E. (1998) : «Lire au collège et au lycée », Actes de la recherche en sciences sociales, 123, p. 25-44.

Baudelot, C., CARTier, E. \& Detrez, C. (1999) : Et pourtant ils lisent, Paris, Éd. Le Seuil.

BAUTIER, E. \& ROCHEX, J.-Y. (1998) : L'expérience scolaire des nouveaux lycéens, Paris, A. Colin.

BAYARD, P. (2002) : Enquête sur Hamlet. Le dialogue de sourds, Paris, Éd. de Minuit.

- (2008) : Comment parler des livres qu'on n'a pas lus ?, Paris, Éd. de Minuit.

Beaude, P.-M., Petitjean, A. \& Privat, J.-M., (dirs) (1996) : La scolarisation de la littérature de jeunesse, Metz, Cresef, (coll. « Didactique des textes »).

BecchetTti-Bizot, C. (2012) : « La question du numérique. Enjeux, défis et perspectives pour l'enseignement des Lettres ", Le Français aujourd'hui, 178, p. 41-51.

BÉHAR, H. \& FAYOLLE, R. (1990) : L'histoire littéraire aujourd'hui, Paris, A. Colin.

BENOIT, J.-P. (1992) : « Des propositions pour observer et développer les compétences interprétatives des élèves ", Pratiques, 76, p. 79-99.

- (1999) : «Le rôle des Instructions Officielles dans la configuration de la discipline Lettres : le cas des concours de recrutement », Pratiques, 101-102, p. 175-190.

BECKER, C. (1986) : La fabrique de Germinal. Édition du dossier préparatoire du roman, Paris/Villeneuve d'Ascq, SEDES/Presses universitaires de Lille. 
BelHadjin, A. (2009) : « Nouvelles approches de la lecture en lycée professionnel », in : M. Butlen \& V. Houdart-Merot (dirs), Interpréter et transmettre la littérature aujourd'hui, Amiens, université de Cergy-Pontoise (coll. Encrage université »), p. 105-121.

BERNARD, J. (2000) : « Discours fictionnel et discours sérieux », in : G. Philippe (dir.), Récits de pensée. Études sur le roman et l'essai, Paris, SEDES, p. 33-43.

BERTRAND, J.-P., DuboIs, J. \& DURAND, P. (1983) : « Approche institutionnelle du premier surréalisme », Pratiques, 38, p. 27-53.

BERTUCCI, M.-M. (2004) : «Enseigner le français : crise de la discipline ou crise de l'identité professionnelle? », in : E. Fraisse \& V.Houdart-Merot (dirs), Les enseignants et la littérature : la transmission en question, Créteil, CRDP de l'Académie de Créteil, p. 135-152.

BESSE, H. (1984) : « Éduquer la perception interculturelle », Le Français dans le monde, 188.

Bessonnat, D. (1990) : « Paroles de personnages : bâtir une progression », Pratiques, 83, p. 5-34.

- (1996) : «Apprendre à écrire une fiche de lecture », Pratiques, 95, p. 95-121.

- (2003) : «Jouer, lire, écrire du théâtre au collège », Pratiques, 119-120, p. 193-220.

BEsSONNAT, D. \& SCHNEDECKER, C. (1990) : «Pour une littérature de seconde main au collège », Pratiques, 67, p. 35-86.

Biguet, C. \& Mougenot, M. (1985) : «Situer les textes », Le Français aujourd'hui, 72, p. 55- 59.

BiKIAlo, S. \& PÉTILlon, S. (2007) : « Une figure de construction entre grammaire et rhétorique : l'ellipse ", Pratiques, 135-136, p. 177-193.

Bilous, D. (2003) : « La mémoire intertextuelle », in : Mémoire et écriture, actes du colloque de l'université de Toulon et du Var, mai 2000, Paris, Champion, p. 323-334.

- (2004) : «Sur la mimoécriture - Essai de typologie », in : Du pastiche, de la parodie et de quelques notions connexes, réunies et présentées par P. Aron, Québec, Éd. Nota bene, p. 103-136.

Bilous, D. \& Bilous, N. (1988) : « La manière deux (pour une didactique du pastiche) », in : C. OriolBoyer. (éd.), La réécriture, Grenoble, Éd. Atelier du texte/Ceditel, p. 125-140.

BISÉNIUS-PENIN, C. (2005) : «Écriture d'invention au lycée et écriture à contraintes », Pratiques, 127-128, p. 209-229.

- (2013a) : «Écriture à contraintes et processus de création à l'université », actes du colloque de Cerisy intitulé Ateliers d'écriture littéraire, 2011, Paris, Hermann, p. 393-408.

- (2013b) : «Place et fonction de l'écriture littéraire dans un cursus universitaire », in : Pratiques d'écriture littéraire à l'Université, Paris, H. Champion, p. 91-104.

BISENIUS-PENIN, C. \& LE Bon, L. (à paraitre) : « Dispositifs culturels et atelier d'écriture au Centre Pompidou-Metz. Médiation pour les publics, médiation par les publics », actes du colloque de Paris, octobre 2013, université Sorbonne Nouvelle-Paris 3.

BISHOP, M.-F. (2005) : « Texte libre et écriture d'invention, quel apport? », Pratiques, 127-128, p. 143-153.

- (2009) : «Le grand absent de l'école primaire », in : M. Butlen \& V. Houdart-Merot (dirs), Interpréter et transmettre la littérature aujourd'hui, Paris, université de Cergy-Pontoise (coll. « Encrage université »), p. 73-92.

- (2010) : «Didactique et perspective historique. À propos d'une recherche sur les écritures de soi à l'école élémentaire ", Pratiques, 145-146, p. 231-248. 
Bishop, M.-F. \& LAcosTe, C. (2007) : « Place et statut du récit de fiction dans les "écrits intermédiaires" à l'école : carnets de lecture, carnets d'expérience et d'observations ", Pratiques, 133-134, p. 155-191.

BoIssinot, A. (2001) : « Les enjeux des disciplines », in : Perspectives actuelles de l'enseignement du français, actes du séminaire national organisé par la direction de l'Enseignement scolaire, ministère de l'Enseignement scolaire, p. 25-38.

BONNEFILLE, V. \& Noyère, A. (2007) : « Lire L'Illiade et l'Odyssée en français et en histoire en classe de sixième. Quelles interactions d'une discipline à l'autre ? », Pratiques, 133-134, p. 221-236.

BorÉ, C. (2000) : « Le brouillon, introuvable objet d'étude? », Pratiques, 105-106, p. 23-49.

- (2004) : « Discours rapportés dans les brouillons d'élèves : vrai dialogisme pour une polyphonie à construire ", Pratiques, 123-124, p. 143-169.

BorowsKI, P. (2003) : « Des gestes pour lire », La Revue française de pédagogie, 142, p. 33-43.

BOUCHARD, R. \& MEYER, J.C. (éds) (1995) : Les métalangages de la classe de français, actes du $6^{\text {e colloque }}$ de la DFLM, sept. 1995, Association internationale pour le développement de la recherche en didactique du français langue maternelle.

Bounoure, A., Declaux, M. \& Pastiaux, J. (1987) : L'enseignement du français vu par des Lycéens et leurs professeurs, Paris, Institu national de recherche pédagogique (coll. « Rapports de recherche »).

BouRdieu, P. (1971a) : « Champ du pouvoir, champ intellectuel et habitus de classe », Scolies, 1, p. 7-26.

- (1971b) : «Le marché des biens symboliques », L'Année sociologique, 22, p. 49-126.

- (1992) : Les règles de l'art. Genèse et structure du champ littéraire, Paris, Éd. Le Seuil.

BOURDIEU, P. \& PASSERON, J.-C. (1964) : Les héritiers : les étudiants et la culture, Paris, Éd. de Minuit. - (1971) : La reproduction : éléments pour une théorie du système d'enseignement, Paris, Éd. Minuit.

BOURGAIN, D. (1989) : Discours sur l'écriture. Analyse des représentations sociales de l'écriture en milieu professionnel, Lille, Atelier national de reproduction des thèses.

BRASSART, D. (1980) : «L'image de l'écrivain chez les enfants d'un CM2 », Pratiques, n27, p. 5-13.

Brassard, D. G., Garcia-Debanc, C., Halté, J.-F., Lebrun, M., Petitjean, A., Legros, G. \& Ropé, F. (dirs) (1990) : Perspectives didactiques en français, actes du colloque de Cerisy Didactique et pédagogie du français, recherches actuelles, Cresef (coll. «Didactique des textes »).

BREMOND, C. \& PAVEL, T. (1999) : De Barthes à Balzac. Fictions d'un critique, critique d'une fiction, Paris, A. Michel.

BRILLANT-ANNEQUin, A. \& MASSOL, J.-F. (dirs) (2005) : Le pari de la littérature. Quelles littératures de l'école au lycée ?, Grenoble, CRDP de l'Académie de Grenoble.

BRONCKART, J.-P. (2013) : «Quelques réflexions pour un redéploiement de la didactique des langues ", in : Les Cahiers Théodile, 5, p. 15-37.

BRUNOT, F. (1895) : «Explication française : observations générales », Revue universitaire, 18 févr., p. 113-128, et 15 mars, p. 263-287.

Bucheton, D. (1999) : « Les postures de lecture des élèves de collèges », in : P. Demougin, \& J.-F. Massol (dirs), Lecture privée et lecture scolaire. La question de la littérature l'école, Grenoble, CRDP de l'Académie de Grenoble, p. 137-150.

- (2008) : L'agir enseignant : des gestes professionnels ajustés, Toulouse, Octarès. 
BuCHETON, D. \& DEZUTTER, O. (dirs) (2009) : Le développement des gestes professionnels dans l'enseignement du français : un défi pour la recherche et la formation, Bruxelles, De Boeck.

Burgos, M. (1992) : « Lecteurs experts, lecteurs “convers". De quelques lectures, en lycée professionnel et ailleurs ", Pratiques, 76, p. 78-94.

- (1993) : «Lectures privées et lectures partagées », Pratiques, 80, p. 78-94.

- (1994) : «La lecture des adolescents : identification et interprétation », L'École des Lettres $1^{\text {er }}$ cycle, 12-13, p. 37-40.

BuRgos, M. \& PRIVAT, J.-M. (1993) : « Le Goncourt des lycéens : vers une socialité littéraire ? », in : M. Poulain (dir.), Lire en France aujourd'hui, Paris, Éd. Le Cercle de la librairie. - (1996) : «Les débats de lecture : pratiques d'appropriation ou construction dialogique des textes ", in : J.-L. Dufays, L. Gemenne \& D. Ledur (1996-2005), Pour une lecture littéraire 2, Bruxelles, De Boeck/Duculot, p. 265-271.

ButLEN, M. (2004) : « Des corpus figés aux corpus éclatés : la littérature pour la jeunesse au service d'une réconciliation sociale ", Le Français aujourd'hui, 145, p. 23-31.

BUtLEN, M. \& HOUDART-MEROT, V. (dirs) (2009) : Interpréter et transmettre la littérature aujourd'hui, actes du colloque, oct. 2008, université de Cergy-Pontoise, Amiens, Encrage université Éd.

CADET, C. (1994) : « La rencontre amoureuse ou comment la lire et comment l'écrire », Pratiques, 81, p. 53-72.

CANVAT, K. (1992) : «Interprétation du texte littéraire et cadrage générique », Pratiques, 76, p. 33-53.

- (1999) : Enseigner la littérature par les genres. Pour une approche théorique et didactique de la notion de genre littéraire, Bruxelles, De Boeck/Duculot.

- (2005) : « De l'enseignement à l'apprentissage de la littérature ou des savoirs aux compétences », in : A. Brillant-Annequin \& J.-F. Massol (dirs), Le pari de la littérature, Quelles littératures de l'école au lycée ?, Grenoble, CRDP de Grenoble, p. 29-41.

Canvat, K. \& Dufays, J.-L. (1994) : « “À la fin de l'envoi, je touche”. Scènes de genres : lecture(s), duels ", Pratiques, 81, p. 73-88.

CANVAT, K. \& VANDERDORPE, C. (1996) : « La fable comme genre : essai de construction sémiotique », Pratiques, 91, p. 27-56.

CAnVAt, K. \& Legros, G. (1997) : « Enseigner la poésie moderne ?», Pratiques, 93, p. 5-29.

CARRÉ, J.-M. \& DEBYSSER, F. (1978) : Jeu, langage, créativité. Les jeux dans la classe de français, Paris, Hachette/Larousse.

CÈBe, S., Goigoux, R., Perez-BACquÉ, M. \& RAguideAu, C. (2012) : Lector et Lectrix (collège), Apprendre à comprendre les textes, Paris, Retz.

CERTEAU, M. de (1980) : L'invention du quotidien. 1. Arts de faire, Paris, Gallimard.

CEYSSON, P. \& QUET, F. (2006) : « Leçon de lecture, construction de sens, construction de soi », Lidil, 33, p. 135-159.

Chabanne, J.-C. (2013) : « Histoire des arts et jeux de langage : comment parler des œuvres ? », Le Français aujourd'hui, 182, p. 55-66.

Chabanne, J.-C., Parayre, M. \& Villagordo, E. (2011) : « Premiers pas dans la parole sur l'œuvre : observer, interpréter et guider les conduites langagières comme compétence professionnelle », Repères, 43, p. 77-102. 
CHABANNE, J.-C., \& DEZUTTER, O. (dirs) (2011) : Les gestes de régulation des apprentissages dans la classe de français, Bruxelles, De Boeck.

ChANFRAUlt-Duchet, M.-F. (2001a) : « Didactique de la littérature : aborder l'analyse des pratiques ", in : M.-J. Fourtanier, G. Langlade \& A. Rouxel, Recherches en didactique de la littérature, Presses universitaires de Rennes, p. 115-122.

- (2001b) : «L'insécurité interprétative des enseignants de lycée », Enjeux, 51-52, p. 63-81.

Chanteau, J.-P. (1990) : « Propositions pour une lecture méthodique du Mariage forcé de Molière », Le Français aujourd'hui, 90, p. 54-61.

CHAPERON, D. \& ReICHLER, C. (2000) : « L'expérience et son traitement dans la science et dans la fiction aux XVIII et XIX ${ }^{\mathrm{e}}$ siècles ", Pratiques, 107-108, p. 35-54.

CHARLE, C. (1978) : La crise littéraire à l'époque du naturalisme, Paris, Presses de l'École normale supérieure.

CHARLES, M. (1985) : L'Arbre et la source, Paris, Éd. Le Seuil.

ChARAudeau, P. (2010) : « Pour une interdisciplinarité "focalisée" dans les sciences humaines et sociales », Questions de communication, 17, p. 195-222.

- (2012) : «Pour une interdisciplinarité focalisée. Réponses aux réactions », Questions de communication, 21, p. 171-206.

Charolles, M. (1990) : « La dissertation quand même », Pratiques, 68, p. 5-16.

Charolles, M., halté, J.-F., Masseron, C. \& Petitjean, A. (1989) : Pour une didactique de l'écriture, Metz, Celted/université de Metz (coll. « Didactiques des Textes).

Charolles, M. \& CombetTes, B. (1999) : « Contribution pour une histoire récente de l'analyse de discours », Langue, Française, 121, p. 76-116.

CHARTIER, A.-M. \& HÉBRARD, J. (1989) : Discours sur la lecture (1889-1990), Centre Georges-Pompidou.

CHARTIER, R. (1998) : « Histoire et littérature », in : Au bord de la falaise. L'Histoire entre certitudes et inquiétude, Paris, A. Michel.

- (2008) : Écouter les morts avec les yeux, Leçons inaugurales du Collège de France, Paris, Collège de France/Fayard.

ChAtTon, A. (2011) : « La poétique des langues de Bernard-Marie Koltès », in : A. Petitjean (éd.), Bernard-Marie Koltès. Textes et contextes, Celted/Université Paul Verlaine-Metz (coll. « Recherches Textuelles »), p. 351-362.

CHERVEL, A. (1986) : Les Auteurs français, latins et grecs au programme de l'enseignement secondaire de 1800 à nos jours, Paris, INRP et Publications de la Sorbonne.

- (1998) : La culture scolaire, Paris, Belin.

- (2002) : « Le baccalauréat et les débuts de la dissertation littéraire (1874-1881) », Histoire de

l'éducation, 94, p. 103-139.

- (2006) : Histoire de l'enseignement du français du XVII $I^{e}$ au XXe siècle, Paris, Retz.

CheVAlard-MAndroux, I. \& TAuveron, C. (1998) : La Lecture de l'œuvre littéraire au lycée, Paris, A. Colin.

CHISS, J.-L. (2011) : « De l'école au collège : apprentissages de la lecture et conceptions de la littérature ", in : A.-M. Godard, E.-M. Havard \& E.-M. Rollat-Levasseur (dirs), L'expérience de lecture et ses médiations. Réflexions pour une didactique, Paris, Riveneuve Éd., p. 101-121. 
- (2012) : «Comprendre et interpréter : réflexion sur la lecture littéraire au cycle 3 », in : L'écrit, la lecture et l'écriture, Paris, Éd. L'Harmattan (coll. « Enfance et langage »), p. 133-144.

CICUREL, F. (2011) : « De l'analyse des interactions en classe de langue à l'agir professoral : une recherche entre linguistique interactionnelle, didactique et théories de l'action », Pratiques, 149/150, p. 41-55.

CITTON, Y. (2007) : Lire, interpréter, actualiser, Paris, Amsterdam.

Clanché, P., Debarbieux, E. \& TestanièRe, J. (dirs) (1994) : La pédagogie Freinet, mises à jour et perspectives, Presses universitaires de Bordeaux.

ClaraC, P. (1964) : L'enseignement du français, Paris, PUF.

CoIRAULT, D. \& DAVID, J. (2011) : « Interactions langue-littérature et approches didactiques. Étude de manuels de troisième du collège », Le Français aujourd'hui, 175, Paris, A. Colin, p. 25-34.

Coirier, P., Gaonac'H, D. \& Passerault, J.-M. (1996) : Psycholinguistique textuelle, Paris, A. Colin.

COLDER, C. \& GAONAC'H, D. (1998) : Lire et comprendre. Psychologie de la lecture, Paris, Hachette.

COLLES, L. (1994) : Littérature comparée et reconnaissance interculturelle, Bruxelles, De Boeck/Duculot.

Colles, L., DufAys, J.-L., FABRY \& G., MAEDER, C. (dirs) (2001) : Didactique des langues romanes. Le développement de compétences chez l'apprenant, Bruxelles, De Boeck/Duculot.

COLTIER, D. (1989) : «Introduction aux paroles de personnages : fonctions et fonctionnement », Pratiques, 64, p. 69-109.

- (1992) : «Iphigénie en $3^{\mathrm{e}:}$ du matériau fictionnel et mythique à la lecture complète de la pièce ou essais sur le théâtre », Pratiques, 74, p. 5-32.

COMBEtTEs, B. (1990) : « Énoncé, énonciation et discours rapporté », Pratiques, 65, p. 97-111.

- (1993) : «Grammaire de phrase, grammaire de texte : le cas des progressions thématiques », Pratiques, 77, p. 43-57.

- (2010) : « Entre théorie et didactique : les débuts de la linguistique de texte en France ", Verbum, 2 (XXXII), p. 219-235.

Compagnon, A. (1998) : Le démon de la théorie, Paris, Éd. Le Seuil.

- (2000a) : «Après la littérature », Le Débat, 110, p. 136-154.

- «Qu'est-ce qu'un auteur ? », cours de licence LLM 316 F2.

- (2000b) : «L'exception française », Textuel, 37, p. 41-52.

CORDONIER, N. (1990) : « La poésie de l'éclectisme critique en situation scolaire : l'exemple de Chantre d'Apollinaire », Pratiques, 67, p. 87-103.

- (1997) : «Les modes de lecture contemporains et leurs perspectives critiques », in : M. NoëlGaudreault (dirs), Didactique de la littérature. Bilan et Perspectives, Québec, Nuit Blanche (coll. « Les Cahiers du centre de recherches en littérature québecoise »), p. 215-232.

CORTIER, C. \& PARPETTE, C. (2006) : « De quelques enjeux et usages historiques du Français fondamental ", Documents pour l'histoire du français langue étrangère ou seconde, 36 (en ligne : http:// dhfles.revues.org/1178).

Coste, D. (1982) : « Apprendre la langue par la littérature ? », in : J. Peytard et al., Littérature et classe de langue, Paris, Hatier (coll. coll. « LAL »), p. 59-73.

COSTE, D., BLANCHET, Ph. dir. (2010) : Regards critiques sur la notion d'interculturalité. Pour une didactique de la pluralité linguistique et culturelle, Paris, Éd. L'Harmattan. 
CROCE-SPINELLI, H. (2007) : Gestes professionnels de l'enseignant et processus interprétatifs des élèves, thèse de doctorat, Toulouse II-Le Mirail.

DABÈNE, M. (1987) : L'adulte et l'écriture. Contribution à une didactique de l'écrit en langue maternelle, Bruxelles, De Boeck.

DAUnAY, B. (1997) : « La paraphrase dans le commentaire de texte littéraire », Pratiques, 95, p. 97-115.

- (1999) : «La lecture littéraire : les risques d'une mystification », Recherches, 30, p. 29-59.

- (2003) : «Les discours sur l'écriture d'invention et les frontières de la discipline », Recherches, 39, p. 39-68.

- (2006) : « Lecture littéraire et disqualification scolaire », Lidil, 33, p. 19-35.

- (2007) : «État des recherches en didactique de la littérature », Revue française de pédagogie, 159, «Politiques et rhétoriques de "l’Ecole juste" avant la Cinquième République ", p. 139-189.

- (2010) : « Français et Littérature : une discipline ou des discipline(s) ? », Le Français aujourd'hui, 168, Paris, A. Colin., p. 23-30.

DAunay, B. \& Denizot, N. (2007) : « Le récit, objet disciplinaire en français ? », Pratiques, $\mathrm{n}^{\circ}$ 133-134, Metz, Cresef, 1-32.

Daunay, B. \& DufaYS, J.-L. (2007) : « Méthodes de recherches en didactique de la littérature », Lettres de l'AIRDF, 40, p. 8-13.

Daunay, B. \& GrossmanN, F. (dirs) (2012) : « Vingt ans de recherches en didactique du français (1990-2010). Quelques aspects des recherches dans les revues », Repères, 46 (en ligne : http:// reperes.revues.org/82).

DE BEAUDRAP, A. R. (dir.) (2004) : Images de la littérature et de son enseignement. Étude réalisée à L'IUFM des Pays de la Loire auprès des PLC de Lettres, Nantes, CNDP-CRDPP.

- (2005) : « La littérature dans les représentations des étudiants et professeurs stagiaires », in : A. Brillant-Annequin \& J.-F. Massol (dirs), Le pari de la littérature. Quelles littératures de l'école au lycée ?, Grenoble, CRDP de l'académie de Grenoble, p. 63-72.

DE BIASI, M. (2011) : Génétique des textes, CNRS Éd.

DeLAS, D. (1983) : « L'enjeu des jeux poétiques », Pratiques, 39, p. 79-100.

DelCAMBRE, I. (1989) : « L'apprentissage du commentaire composé : comment innover ?», Pratiques, 63, p. 13-36.

- (1990) : « De l'argumentation à la dissertation. Analyse d'une démarche d'apprentissage », Pratiques, 68, p. 69-88.

DeLESALLE, S. (1970) : «L'explication de textes, fonctionnement et fonction », Langue française, 7 , p. 87-95.

- (1979) : «Primaire-secondaire. Le paradigme et le syntagme », Pratiques, 22-23, p. 129-145.

Demougin, P. \& MAssol, J.-F. (1999) : Lecture privée et lecture scolaire, Grenoble, Centre régional de documentation de l'académie de Grenoble.

DelfaU, G. \& Roche, A. (1977) : Histoire/Littérature, histoire et interprétation du fait littéraire, Paris, Éd. Le Seuil.

DeMougin, P. \& MASsOL, J.-F. (dir.s (1999) : Lecture privée et lecture scolaire, Grenoble, CRDP de l'académie de Grenoble.

Denhière, G. \& LEgros, D. (1983) : « Comprendre un texte », Revue française de pédagogie, 75, Paris, INRP, p. 85-98. 
DENIS, B. (2000) : Littérature et engagement, Paris, Éd. Le Seuil.

Denizot, N. (2005) : « L'écriture d'invention au lycée : écriture des genres ou nouveau genre scolaire? ", Pratiques, 127/128, p. 31-44.

- (2010) : «Genres littéraires et genres textuels dans la discipline français », Pratiques, 145-146, p. 211- 230 .

- (2013) : «La dissertation, un genre scolaire argumentatif ? Perspective historique », Pratiques, 157-158, p. 195-205.

DERONNE, C. (2011) : « Former des enseignants à exprimer leur expérience singulière de l'œuvre littéraire ou plastique », Repères, 43, p. 103-126 (en ligne : http://reperes.revues.org/222).

DERRIDA, J. (1967) : L'écriture et la différence, Paris, Éd. Le Seuil.

- (1972) : La dissémination, Paris, Éd. Le Seuil.

DE SINGLY, F. (1989) : Lire à 12 ans, Paris, Nathan.

DESCOTES, M. (1989) : La lecture méthodique, Toulouse, CRDP de Toulouse.

- (1990) : «La lecture méthodique : un problème de représentations ? », Le Français aujourd'hui, 90, p. 31-39.

DÉTRIE, C. (2005) : « La dynamique idiolectisante, entre singularisation et réitération », Cahiers de praxématique, 44 , p. 51-75.

DeVelay, M. (1995) : De l'apprentissage à l'enseignement, pour une épistémologie scolaire, Paris, ESF.

DEWEY, J. (1997 [1934]): Arts as Experience, Carbondale, Southern Illinois University Press.

DEZUTTER, O. (2002) : «Genre épistolaire et positionnement du scripteur : une liberté sous contrainte », Pratiques, 113-114, p. 83-94.

DezutTeR, O. \& Dufays, J.-L. (1999) : « L'apprentissage de l'écriture au secondaire dans les instructions belges de l'après-guerre », Pratiques, 101-102, p. 205-210.

DieU, A.-M., DRUART, G. \& RENARD, E. (1995) : L'enseignement du français : quelle histoire! Le cours de langue au niveau du secondaire en Belgique francophone de 1945 à 1990, Bruxelles-Lier, Van In. DOLZ, J., ROSAT, M.-C. \& SCHNEUWLY, B. (1991) : «Élaboration et évaluation de deux séquences didactiques relatives à trois types de textes ", Le Français aujourd'hui, 93, A. Colin, p. 37-47.

DOLZ, J. \& OlLAGNIER, E. (dirs) (2002) : L'énigme de la compétence en éducation, Bruxelles, De Boeck.

DonNAT, O. (2009) : «Les pratiques culturelles des Français à l'ère numérique. Éléments de synthèse 1997-2008 », Cultures Études, 5.

DOUBRowSKY, S. \& TODOROV, T. (éds) (1971) : L'enseignement de la littérature, actes du colloque de Cerisy-la-Salle, Paris, Plon.

DuBET, F. (1991) : Les lycéens, Paris, Éd. Le Seuil.

DuBoIS, J. (1978) : L'institution de la littérature, Nathan, Labor.

- (1980) : «Lecture sociologique de l'histoire littéraire », Pratiques, numéro spécial colloque de Cerisy intitulé « Pour un nouvel enseignement du français », p. 85-94.

Duchesne, A. \& Leguay, T. (1985) : Petite fabrique de littérature, Paris, Magnard.

Dufays, J.-L. (1997) : « Lire au pluriel. Pour une didactique de la diversité des lectures à l'usage des 14-15 ans ", Pratiques, 95, p. 31-52.

- (2001a) : «Quelles(s) méthodologie(s) pour les recherches en didactique de la littérature ? ", Enjeux, 51-52, Namur, p. 7-39. 
- (2001 b) : «Didactique du français et réflexion épistémologique : où en sommes nous, que voulons nous ? ", in : M. Marquillo-Larrruy (éd.), Questions d'épistémologie en didactique du français, langue maternelle, langue seconde, langue étrangère, s.l., Les Cahiers FORELL/université de Poitiers, p. 309-318.

- (2005) : «Les lectures littéraires : évolution et enjeux d'un concept », in : A. Brillant-Annequin \& J.-F. Massol (dirs), Le pari de la littérature. Quelles littératures de l'école au lycée ?, Grenoble, CRDP de l'académie de Grenoble, p. 185-195.

- (2006) : «La lecture littéraire, des "pratiques du terrain" aux modèles théoriques », Lidil, 33, université de Grenoble, p. 79-101.

- (2007) : « De la discipline déclarée à la discipline apprise : un an d'observation de deux enseignantes de français et de leurs élèves en classe de $5^{\mathrm{e}}$ secondaire ", in : E. Falardeau, C. Fisher, C. Simard, \& N. Sorin, N. (dirs), La didactique du français. Les voies actuelles, Québec, Presses universitaires de Laval, p. 63-82.

- (2010a) : Stéréotype et lecture, Mardaga (1994), Berne, P. Lang.

- (2010b) : «Discontinuités dans l'enseignement de la littérature en Belgique francophone », Le Français aujourd'hui, 168, Paris, A. Colin, p. 33-42.

- (2011) : "Quel enseignement de la lecture et de la littérature à l'heure des "compétences" ?", Pratiques, 149-150, p. 227-248.

DufAYS, J.-L. \& GEMENNE, L. (1995) : « De l'analyse textuelle à l'appropriation personnelle des textes ", Le Français aujourd'hui, 112, p. 72-79.

Dufays, J.-L., GEMENNE, L. \& Ledur, D. (1996a) : Pour une lecture littéraire 1. Approches historique et théorique. Propositions pour la classe de français, Bruxelles, De Boeck/Duculot.

- (1996 b) : Pour une lecture littéraire 2. Bilan et confrontations, actes du colloque de Louvain-laneuve, mai 1995, Bruxelles, De Boeck/Duculot.

DufAYS, J.-L. \& RosiER, J.-M. (1999) : « La littérature dans les programmes de français de l'enseignement secondaire en Belgique : essai de mise en perspective historique ", Enjeux, 43-44, Namur, Faculté Notre-Dame de la Paix, p. 22-143.

Dufiet, J.-P. \& Petitjean, A. (2013) : «Candide de Jean Tardieu : étude sémio-linguistique d'une adaptation de l'œuvre de Voltaire ", in : Approches linguistiques des textes dramatiques, Paris, Classiques Garnier.

DuhAmEL, B. (1993) : «S'entretenir de leurs lectures », Pratiques, 80, p. 56-77.

- (1984) : « DES LOUPS ET DES LECTEURS OU : quand une équipe d'enseignants essaie d'observer les compétences en lecture des élèves au CM2 et en $6^{\mathrm{e}}$ ", Pratiques, 44, p. 70-82.

DUHAMEL, B. \& MASSERON, C. (1987) : « Trois machines romanesques », Pratiques, 55, p. 100-125.

DUMORTIER, J.-L. (2006) : « Conduite esthétique, jugement critique et écriture de soi », Repères, 34, p. $185-214$.

DUMORTIER, J.-L., DisPy, M. \& VAN BEVEREn, J. (2011) : « Pour le discours incitatif. Qu'apprendre aux maitres à dire à leurs élèves pour inciter ces derniers à lire les œuvres littéraires en leur prêtant la qualité d'attention requise par l'intention artistique », Repères, 43, p. 31-52.

DUPONT, D., REUTER, Y. \& RosIER, J.-M. (1988) : Manuel d'histoire littéraire. Introduction au champ littéraire contemporain, Bruxelles, De Boeck/Duculot.

- (1990) : Histoire littéraire, Bruxelles, De Boeck/Duculot.

DUPONT, D. \& ROSIER, J.-M. (1983) : « Faire de l'histoire littéraire autrement : Le cas Montaigne », Pratiques, 38, p. 5-82. 
ECO, U. (1985 [1979]) : Lector in fabula ou la coopération interprétative dans les textes narratifs, trad. de l'italien, Paris, Grasset.

- (2011-2013) : Confessions d'un jeune romancier, Paris, Grasset.

ElALouf, M.-L. (2009) : « Les limites de l'interprétation : le rôle dévolu à la syntaxe », in : M. Butlen \& V. Houdart-Merot (dirs), Interpréter et transmettre la littérature aujourd'hui, université de CergyPontoise, Encrage université Éd., p. 135-151.

ELGIN, C. (1992) : «Comprendre l'art et la science », in : R. Pouivet (éd.), Lire Goodman, Combas, Éd. de l'Éclat.

EMERY-BRUNEAU, J. (2011) : " Parcours de sujets-lecteurs et transformations du rapport à la lecture littéraire : d'une pratique singulière à quelles intentions didactiques? », in : R. Goigoux, M.-C. Pollet (dirs), Didactiques de la lecture de la maternelle à l'université, Namur, Presses universitaires de Namur, p. 158-181.

Etchegoyen, A. (1990) : Le capital Lettres. Des littéraires pour l'entreprise, Paris, F. Bourin.

ÉTIENNE, B. (2011) : « De la dérive techniciste à la tectonique des textes », Le Français aujourd'hui, 175, Paris, A. Colin, p. 11-24.

FABRE, D. (1993) : Par écrit. Ethnologie des écritures quotidiennes, Paris, Éd. de la Maison des sciences de l'homme.

FALARDEAU, E. (2003) : « Pour une mise à distance des stéréotypes socioculturels : l'exemple de L'Écume des jours ", Pratiques, 117-118, p. 165-170.

FALARDEAU, E., FiSHER, C., SimARD, C. \& SORIN, N. (dirs) : La didactique du français. Les voies actuelles, Québec, Presses universitaires de Laval.

FALARDEAU, E. \& SIMARD, D. (2011) : « La prise en compte du rapport à la culture dans le discours des enseignants sur les œuvres littéraires », Repères, 43, p. 53-73.

FAYOL, M. et al. (1992) : Psychologie cognitive de la lecture, Paris, PUF.

FisH, S. (1980-2007) : Quand lire c'est faire. L'autorité des communautés interprétatives, Paris, Éd. Les Prairies ordinaires.

FonTANille, J. (1999) : Sémiotique et littérature, Essais de méthode, Paris, PUF.

FouCAult, M. (1969) : L'archéologie du savoir, Paris, Gallimard.

FoURNIER, J.-M. \& VECK, B. (1997) : « La lecture littéraire. Un dispositif articulé du collège au lycée », Pratiques, 95, p. 7-30.

FOURTANIER, M.-J., LANGLADE, G. \& ROUXEL, A. (2001) : Recherches en didactique de la littérature, Rennes, Presses universitaires de Rennes.

FRAISSE, E. (1985) : « L'invention d'une littérature scolaire : les manuels de morceaux choisis de 1872 à 1923 ", Études de linguistique appliquée, 59, p. 102-109.

- (1997) : Les anthologies en France, Paris, PUF.

FraisSe, E. \& HOUdART-MEROT, V. (dirs) (2004) : Les enseignants et la littérature : la transmission en question, actes du colloque de Cergy-Pontoise, SCEREN-CRDP de l'académie de Créteil.

FRIOT, B. (1996) : « La littérature de jeunesse au collège : ouverture et réticences dans les textes officiels ", in : P.-M. Beaude, A. Petitjean \& J.-M. Privat (dirs), La scolarisation de la littérature de jeunesse, Cresef (coll. « Didactique des textes », p. 49-59.

GADAMER, H-G. (1976 [1960]) : Vérité et méthode, Paris, Éd. Le Seuil. 
GAGNÉ, G., LAZURE, R., SPRENGER-CHAROLLES, L. \& ROPÉ, F. (1989) : Recherches en didactique et acquisition du français langue maternelle (1970-1984), t. 1, Cadre conceptuel, thésaurus et lexique des mots-clés ; t. 2, Répertoire bibliographique, Bruxelles/Paris, De Boeck/Wesmael/Editions universitaires.

GAGNON, J.-C. (1985) : « La lecture du texte littéraire comme document authentique et la culture langagière ", in : M. Thérien \& G. Fortier (dirs), Didactique de la lecture au secondaire, Québec, VilleMarie, p. 201- 220.

GALLY, M. (2006) : Le bûcher des humanités. Le sacrifice des langues anciennes et des lettres est un crime de civilisation, Paris, A. Colin.

GenEtTe, G. (1969) : « Rhétorique et enseignement », in : Figures II, Paris, Éd. Le Seuil, p. 23-42.

- (1982) : Palimpsestes, la littérature au second degré, Paris, Éd. Le Seuil.

- (1991) : Fiction et diction, Paris, Éd. Le Seuil.

- (1997) : L'œuvre de l'art 2. La relation esthétique, Paris, Éd. Le Seuil.

GeNINASCA, J. (1997) : La parole littéraire, Paris, PUF.

GODARD, A. (2011) : « Des stratégies de lecture à l'expérience des lecteurs », in : A.-M. Godard, E.M., Havard, E.-.M., Rollat-Levasseur (dirs), L'expérience de lecture et ses médiations. Réflexions pour une didactique, Paris, Riveneuve Éd., p. 19-36.

Goigoux, R. (2000) : Les élèves en grandes difficultés de lecture et les enseignements adaptés, Paris, CNEFEI.

GolDENSTEIN, J.-P. (1976) : «"Mateo Falcone” (II) : travaux d'approche », Pratiques, 11-12, p. 94-108. - (1978a) : « Du monde entier au cœur du mot. Propositions de travail sur le recueil de poèmes de Blaise Cendrars », Pratiques, 20, p. 3-20.

- (1978b) : «La pédagogie du jeu poétique », Pratiques, 21, p. 48-56.

- (1983a) : « Enseigner la littérature ?», Pratiques, 38, p. 3-8.

- (1983b) : « Le tri de la postérité : approches de quelques mécanismes », Pratiques, 38, p. 11-25.

- (1990) : Entrées en littérature, Paris, Hachette.

Goodman, N. (1990 [1968]) : Langages de l'art. Une approche de la théorie des symboles, trad. de l'anglais, Nîmes, J. Chambon.

Goulemot, J.-M. (1979) : « Histoire littéraire, science des œuvres ? ", in : Le Goff et al., La Nouvelle histoire, Paris, CEPL (coll. Les Encyclopédies du savoir moderne).

- (1985) : « De la lecture comme production de sens », in : Pratiques de lecture, Paris, Rivages.

Gouvard, J.-M. (dir.) (2005) : De la langue au style, Lyon, Presses universitaires de Lyon.

GRANGE, M., \& LEROUX, M. (2005) : « La pédagogie sans dessus dessous. Les programmes de français de collèges ", Le Débat, 135, p. 22-36.

GREIMAS, A.-J. (1976) : « Sémiotique narrative et textuelle », Pratiques, 11-12, p. 5-2.

- (1987) : De l'Imperfection, Périgueux, P. Fanlac.

GRÉSILLON, A. (1988) : « Les manuscrits littéraires : le texte dans tous ses états », Pratiques, 57,

p. 107-122.

- (1994) : Eléments de critique génétique, Paris, PUF.

GRICE, H.P. (1979 [1975]) : « Logique et conversation », trad. de l'anglais, Communications, 30, p. 57-72.

GUERNIER, M.-C. (2006) : «Variations autour de ce qu'il doit rester d'une lecture. Que nous dit la fiche de lecture?", Lidil, 33, p. 103-115. 
GUIRAUD, P. (1963) : Essais de stylistique, Paris, Klincksieck.

HalTÉ, J.-F. (1978) : « Je n'écris pas sur les oiseaux », Pratiques, 21, p. 35-47.

- (1985) : L'enseignement du français dans le travail en projet, thèse Nouveau Régime, université de Besançon.

- (1987) : «Écriture, littérature, formation », Cahiers du CRELEF, 25, p. 81-103.

- (1992) : La didactique du français, Paris, PUF (coll. « Que sais-je ?»).

- (dir.) (1991) : Inter-Actions, Metz, université de Metz (coll. « coll. Didactiques des textes »).

- (1995-2005) : «Interaction : une problématique à la frontière », in : de J.-L. Chiss, J. David, \& Y. Reuter (dirs), Didactique du Français. Fondements d'une discipline, Bruxelles, De Boeck, p. 61-75.

Halté, J.-F., Michel, R. \& Petitjean, A. (1974) : « Essai d'analyse structurale du “Chat noir” d’E. Poe. Pour une application pédagogique », Pratiques, 1- 2, p. 5-28.

Halté, J.-F. \& Petitjean, A. (1974) : « Pour une théorie de l'idéologie d'un manuel scolaire, Le Lagarde et Michard : le cas Diderot », Pratiques, 1/-2, p. 43-64.

HAMBURGER, K. (1977) : Logique des genres littéraires, Paris, Éd. Le Seuil.

Hamon, P. (1973) : « Un discours contraint », Poétique, 16, p. 411-445.

- (1984) : Texte et idéologie, Paris, PUF (coll. « Écriture »).

HeINICH, N. (1998) : Ce que l'art fait à la sociologie, Paris, Éd. de Minuit.

- (2000) : Être écrivain, Paris, Éd. La Découverte.

HERSCHBERg PIERROT, A. (2005) : Le style en mouvement. Littérature et art, Paris, Belin.

- (2007) : «Style et genèse des œuvres », Pratiques, 135-136, p. 163-176.

HOUDART-MÉROT, V. (1998) : La culture littéraire au lycée depuis 1880, Rennes, PUR/ADAPT Éd.

- (2003) : Interpréter et transmettre la littérature aujourd'hui, Amiens, Encrage Université Éd.

- (2004a) : «Intentions et ruses de la transmission : tentative de définition », in : E. Fraisse, \&

V. Houdart-Merot (dirs), Les enseignants et la littérature : la transmission en question, Créteil, CRDP de l'académie de Créteil, p. 17-30.

- (2004b) : Réécriture et écriture d'invention au lycée, Paris, Hachette.

- (2004c). « De la critique d'admiration à la lecture "scriptible" ", in : A. Rouxel \& G. Langlade (dirs), Le sujet lecteur. Lecture subjective et enseignement de la littérature, Rennes, PUR, p. 223-232.

- (2013) : «L'émergence conflictuelle des ateliers d'écriture dans les universités françaises », in :

V. Houdart-Mérot \& C. Mougenot (dirs), Pratiques d'écriture littéraire à l'université, Paris,

H. Champion, p. 41-60.

HoudART-MÉRot, V. \& MOUgenot, C., (dirs) (2013) : Pratiques d'écriture littéraire à l'université, actes du colloque de Cergy-Pontoise, 2010, Paris, H. Champion.

Houdebine, J.-L. (1971) : « Sur l'idéologie du “Lagarde et Michard”, Littérature/Science/idéologie, 1, p. 10-16.

Hubat-Blanc, A.-M. (1996) : «Quelques exemples et quelques analyses de résistances d'élèves à l'étude littéraire », in : J.-L. Dufays, L. Gemenne \& D. Ledur, Pour une lecture littéraire 2. Bilan et confrontations, actes du colloque de Louvain-la-neuven, mai 1995, Bruxelles, De Boeck/Duculot, p. 319-327.

HuYHN, J.-A. (2004) : « Écriture d'invention et "identité” du sujet lecteur », in : A. Rouxel \& G. Langlade (dirs) (2004), Le sujet lecteur. Lecture subjective et enseignement de la littérature, Rennes, PUR, p. 305-316.

IDT, G. (1977) : « Pour une "histoire littéraire" tout de même », Poétique, 30, p. 167-174. 
INISAN, J.-F., CHARDon, O. \& RoELENS, F. (2007) : « Écrire le journal intime d'un personnage du XVIII ${ }^{\mathrm{e}}$ siècle. Une expérience interdisciplinaire en classe de quatrième », Pratiques, 133-134, p. 193-220.

ISER, W. (1986 [1976]) : L'acte de lecture. Théorie de l'effet esthétique, trad. de l'allemand, Paris, Mardaga.

JAKOBSON, R. (1963 [1960]) : «Linguistique et poétique », in : Essais de linguistique générale, Paris, Éd. de Minuit, p. 209-248.

JARRETY, M. (2000) : Propositions pour les enseignements littéraires, Paris, PUF.

JAUBERT, A. (1990) : La lecture pragmatique, Paris, Hachette.

JAUBERT, M. \& REBIÈRE, M. (2011) : « Des gestes professionnels pour aider à mieux comprendre les textes au collège ", in : J.-C. Chabanne \& $\mathrm{O}$. Dezutter, $\mathrm{O}$. (dirs), Les gestes de régulation des apprentissages dans la classe de français, Bruxelles, De Boeck, p. 107-128.

JAUSS, H.-R. (1978) : Pour une esthétique de la réception, Paris, Gallimard.

JENNY, L. (1990) : La parole singulière, Paris, Belin.

JEY, M. (1998) : La littérature au lycée : invention d'une discipline (1880-1925), Metz, université Paul Verlaine-Metz (coll. « «Recherches textuelles » ).

- (1999) : «La littérature au lycée en France à partir des textes officiels de 1947 à 1981 », Enjeux, 43-44, p. 144-165.

- (2000) : «Crise du français et réforme de l'enseignement secondaire (1902-1914) », Études de linguistique appliquée, 118, p. 163-177.

JooLE, P. (2009) : « Le carnet de "lecteur littéraire" », in : M. Butlen \& V. Houdart-Merot (dirs), Interpréter et transmettre la littérature aujourd'hui, université de Cergy-Pontoise, Encrage université, p. 291-306.

JORDY, J. (2009) : « Au lycée : interprétation collective et texte du lecteur », in Butlen, M. \& Houdart-Merot, V., dir (2009) : Interpréter et transmettre la littérature aujourd'hui, Amiens, université de Cergy-Pontoise/Encrage Éd., p. 93-103.

JoRRO, A. (2002) : Professionnaliser le métier d'enseignant, Issy-les-Moulineaux, ESF.

JoRRo, A. \& CROCÉ-SPINELLI, H. (2010) : « Le développement des gestes professionnels en classe de français. Le cas de situations de lecture interprétative ", Pratiques, 145-146, p. 125-140.

JosTE, A. (2002) : Contre-expertise d'une trahison, la réforme du français au lycée, Paris, Éd. Mille et une nuits.

JouVE, V. (1992) : L'effet-personnage dans les romans, Paris, PUF.

- (1993) : La lecture, Paris, Hachette.

- (2001) : Poétique des valeurs, Paris, PUF (coll. «Écriture »).

- (2003) : «La dénonciation du malentendu. Enquête sur les discours de réfutation », in:

B. Clément \& M. Escola (dirs), Le Malentendu. Généalogie du geste herméneutique, Vincennes, Presses universitaires de Vincennes, p. 191-201.

- (2005) : L'expérience de lecture, Paris, Éd. L'Improviste.

- (2010) : Pourquoi étudier la littérature, Paris, A. Colin.

- (2011) : «Quel statut accorder à la lecture dans l'enseignement de la littérature ? ", in : A.-

M. Godard, A.-M. Havard \& E.-M. Rollat-Levasseur (dirs), L'expérience de lecture et ses médiations. Réflexion pour une didactique, Paris, Riveneuve Éd., p. 37-47.

JURT, J. (1980) : La réception de la littérature par la critique journalistique. Lectures de Bernanos (1926-1936), Paris, J.-M. La Place. 
- (1983) : « “L’Esthétique de la réception”. Une nouvelle approche de la littérature ?», Lettres Romanes, 3, Université Catholique de Louvain.

KeRBRAT-ORECHIONNI, C. (1980) : L'énonciation, de la subjectivité dans le langage, Paris, A. Colin.

KirPalani, M.-C. (2000) : « Approche d'un genre : la nouvelle », Pratiques, 107-108, p. 145-204.

KLINKERBERG, J.-M. (1998) : « L'analyse institutionnelle de la littérature en Belgique francophone : où en est-on?", Textyles, 15, « L'institution littéraire », p. 7-11.

KuENTZ, P. (1972) : «L'envers du texte », Littérature, 7, p. 3-26.

- (1974) : « Le tête à texte », Esprit, 12.

LABROSSE, C. (1985) : Lire au XVIII siècle. La Nouvelle Héloïse et ses lecteurs, Lyon, PUL.

Lacoue-Labarthe, P. \& Nancy, J.-L. (1978) : L'absolu littéraire, Paris, Éd. Le Seuil.

LAFARGE, C. (1983) : La valeur littéraire. Figuration littéraire et usages sociaux des fictions, Paris, Fayard.

LAFONTAINE, D. (2002) : « Les compétences à l'épreuve : l'évaluation en lecture », Enjeux, 55, p. 54-66.

LAHIRE, B. (1993) : « Lectures populaires : les modes d'appropriation des textes », Revue française de pédagogie, 104, p. 17-26.

- (1997) : Les manières d'étudier, Paris, Documentation Française.

- (1998) : «De l'expérience littéraire : lecture, rêverie et actes manqués », in : L'Homme pluriel, Paris, Nathan.

- (2006) : La condition littéraire, Paris, Éd. La Découverte.

LAMrany El Alaoui, R. (2005) : « Le support littéraire. Entre réception et écriture d'invention. Présentation de onze productions d'élèves marocains en fin de scolarité », thèse, université Paul Verlaine-Metz.

LANGLADE, G. (2001a) : « La lecture littéraire : savoirs, réflexion et sentiments », in : Perspectives actuelles de l'enseignement $d u$ français, actes du séminaire national organisé par la direction de l'Enseignement scolaire, ministère de l'Enseignement scolaire, p. 143-152.

- (2001b) : «Et le sujet lecteur dans tout ça ? », Enjeux, 51-52, p. 53-62.

- (2004a) : «Sortir du formalisme, accueillir les lecteurs réels », Le Français aujourd'hui, 145, p. 85-96.

- (2004b) : « Le sujet lecteur auteur de la singularité de l'œuvre », in: A. Rouxel \& G. Langlade (dirs), Le sujet lecteur. Lecture subjective et enseignement de la littérature, Rennes, PUR, p. 81-91.

LANE, P. (1984) : « Propositions pour évaluer les compétences de lecture des élèves », Pratiques, 44, p. 83- 89 .

LANSON, G. (1902) : «Contre la rhétorique et les mauvaises humanités », in : L'Université et la société moderne, Paris, A. Colin.

- (1903) : «Les Études modernes dans l'enseignement secondaire », in : L'Éducation de la démocratie, Paris, F. Alcan.

- (1904) : "L'histoire littéraire et la sociologie », Revue de métaphysique et de morale, XII, p. 621-642.

Le Cadre européen commun de référence pour les langues (2008) : Apprendre, Enseigner, Évaluer, Paris, Didier.

LAPARRA, M. (1991) : « Problèmes de lecture posés par l'écriture des textes historiques à visée didactique ", Pratiques, 69, p. 97-124.

- (2010) : « Lectures lycéennes du Colonel Fabert », in : J-M. Privat \& M. Scarpa (dirs), Horizons 
ethnocritiques, Nancy, PUN.

- (2011) : « Le pays où "les rats ne mangent pas les chats" ou l'histoire de Gavroche et de l'éléphant », Pratiques, 151-152, p. 207-225.

LAUDET, P. (2009) : « Place et enjeux de la littérature dans les nouveaux programmes des collèges ", in : actes des séminaires inter-académiques sur les nouveaux programmes de lycée, mars 2010, Eduscol, p. 2-11.

- (2011) : «L'explication de texte littéraire : un exercice à revivifier », intervention au séminaire inter-académique sur les nouveaux programmes de français au collège, du 20 janvier au 7 mars 2009, Eduscol.

LEBRUN, M. (2004) : « L'émergence et le choc des subjectivités de lecteurs de la maternelle au lycée grâce à l'espace interprétatif ouvert par les comités de lecture ", in : A. Rouxel, \& G. Langlade (dirs), Le sujet lecteur. Lecture subjective et enseignement de la littérature, Rennes, PUR, p. 329-341

LEBrun, M. \& Roy, M. (1999) : « Langue, discours, littérature. Panorama de l'enseignement de la littérature au Québec, du Rapport Parent aux réformes des années 1980 », Enjeux, 43-44, p. 166-190.

LeCLAire-HALTÉ, A. (2003) : « Textes d'action et valeurs dans les robinsonnades : étude et pistes didactiques », Pratiques, 117-118, p. 77-115.

- (2004a) : Robinsonnades et valeurs en littérature de jeunesse contemporaine, Metz, université de Metz (coll. Didactiques des textes).

- (2004b) : " Un album "polyphonique" au cycle III : Histoire à quatre voix d'Anthony Browne ", Pratiques, 123-124; p. 113-142.

- (2010) : «Interactions didactiques et valeurs : échanges autour du message d'un album. Études de cas ", Pratiques, 147-148, p. 155-172.

LEDUR, D. (1996) : « Lecture littéraire et enseignement professionnel : faut-il former des "héritiers" ? », in : J.-L. Dufays, L. Gemenne \& D. Ledur, Pour une lecture littéraire 2. Bilan et confrontations, actes du colloque de Louvain-la-Neuve, mai 1995, Bruxelles, De Boeck/Duculot, p. 328-338.

LEenhardT, J. \& JószA, P. (1999 [1982]) : Lire la lecture, rééd., Paris/Montréal, Éd. L’Harmattan.

LEGOFF, F. (2004) : « Métadiscours en écriture d'invention et modes d'investissement du sujet lecteur ", in : A. Rouxel \& G. Langlade (dirs), Le sujet lecteur. Lecture subjective et enseignement de la littérature, Rennes, PUR, p. 293-304.

- (2005) : «L'écriture d'invention au lycée : ce que disent les professeurs de leurs pratiques », Pratiques, 127-128, p. 60-74.

- (2009) : « Enquête sur un écrit de savoir au lycée : la dissertation littéraire », Pratiques, p. 143-144, p. 11-126.

Legros, G., Monballin, M. \& VANDER Brempt, M. (1991) : « Le cercle des poètes rebattus », Enjeux, 24, p. 5-24.

LORIES, D. (éd.) (1988) : Philosophie analytique et esthétique, Paris, Méridien Klincksieck.

LOTMAN, I. (1973) : La structure du texte artistique, Paris, Gallimard.

LOUICHON, B. ( 2008) : « Enquête sur le rapport des enseignants aux programmes de 2002 et les pratiques d'enseignement de la littérature au cycle 3 ", Repères, 37, p. 51-68.

MACHEREY, P. (1970) : Pour une théorie de la production littéraire, Paris, F. Maspero.

- (2010) : «Science, philosophie, littérature », Textuel, 37, p. 133-142. 
MAINGUENEAU, D.(1986) : Eléments de linguistique pour le texte littéraire, Paris, Dunod.

- (1999) : «Réflexions sur la "grammaire de discours" au collège », Le Français aujourd'hui, 128, p. 20-28.

- (2003) : « Les apports de l'analyse du discours à la didactique de la littérature », Le Français aujourd'hui, 141, p. 73-82.

- (2004) : Le discours littéraire. Paratopie et scène d'énonciation, Paris, A. Colin.

- (2011) : " Lecture et surlecture », in : A. Godard, A.-M. Havard \& E.-M. Rollinat-Levasseur (dirs), L'expérience de lecture et ses médiations, Paris, Riveneuve Éd., p. 49-60.

- (2013) : «Genres de discours et web : existe-t-il des genres web ?», in : C. Barats (dir.), Manuel d'analyse du web, Paris, A. Colin, p. 74-93.

MANESSE, D. \& GRellet, I. (1994) : La littérature du collège, Paris, INRP/Nathan.

MANSUY, M., (éd.) (1977) : L'enseignement de la littérature : crise et perspectives, actes du colloque de Strasbourg, déc. 1975, Paris, Nathan.

MARGHESCOU, M. (1974) : Le concept de littérarité. Essai sur les possibilités théoriques d'une science de la littérature, La Haye/Paris, Mouton ; $2^{\mathrm{e}}$ éd. revue : (2009) : Le concept de littérarité. Critique de la métalittérature, Paris, Kimé.

MARLAIR, S. \& DufAYS, J.-L.( 2011) : « (Faire), découvrir un texte littéraire en classe : ce que les variations du geste enseignant révèlent de la relation esthétique au texte ", in : J.-C. Chabanne \& O. Dezutter (dirs), Les gestes de régulation des apprentissages dans la classe de français, Bruxelles, De Boeck, p. 83-106.

MARQUILLO-LARRUY, M. (éd.) (2001) : « Questions d'épistémologie en didactique du français », Les Cahiers FORELL, 15.

MAsSeron, C. (1980) : «L'image de l'écrivain dans le secondaire », Pratiques, 27, p. 14-22.

- (1990) : «Élaboration d'un texte long : l'exemple du genre fantastique », Pratiques, 86, p. 3-56.

- (1996) : « Séquences didactiques ? (faire) faire écrire des récits de création en $5^{\mathrm{e}}$ », Pratiques, 92, p. 5-55.

- (1994) : «Écrire des récits d'énigme criminelle », Pratiques, 83, p. 35-77.

- (2003a) : «Conseiller un film d'horreur. Justifications de jugements de valeurs dans quelques copies de seconde », Pratiques, 117-118, p. 219-240.

- (2003b) : «Valeurs éducatives et systèmes de valeurs à travers quelques écrits d'apprentissage », Pratiques, 117-118, p. 145-164.

MASSERON, C. \& SCHNEDECKER, C. (1988) : «Le mode de désignation des personnages », Pratiques, 60, p. 98-123.

Masseron, C. \& Petitjean B. (1979) : « Pour une définition du personnage : l'exemple de Germinal », Pratiques, 22-23, p. 69-96.

MASSOL, J.-F. (2001) : Euvres intégrales pour les lycées. Genres et titres contemporains, Paris/Grenoble, Delagrave/CRDP de Grenoble.

- (2004) : De l'institution scolaire de la littérature française (1870-1925), Paris, Éd. littéraires et linguistiques de l'université de Grenoble.

- (1999) : Lecture privée et lecture scolaire, Grenoble, CRDP de l'académie de Grenoble.

MAтнis, G. (2001) : «Le réglage de la distance dans la lecture littéraire : catharsis et identification esthétique ", Enjeux, 51-52, p. 83-92.

MAuger, G., PoliaK, C. \& PudAl, B. (1999) : Histoires de lecteurs, Paris, Nathan. 
MELANÇON, J. (1993) : La littérature au CEGEP (1968-1978). Le statut de la littérature dans l'enseignement collégial, Québec, Éd. Nuit Blanche.

MÉNAND-DoumAZANE, F. (2011) : «G. Flaubert, C. F. Ramuz : lectures en spirale. Ethnocritique du texte littéraire », Pratiques, 151-152, p. 187-206.

MERLEAU-PonTY, M. (1960) : « Le langage indirect et les voix du silence », in : Signes, Paris, Gallimard, p. 96-97.

Michel, R. (1997) : «Étude d'une forme fixe. Le sonnet », Pratiques, 93, p. 117-124.

- (1998) : « Lecture méthodique ou méthode de lecture à l'usage des élèves de lycée : un objet didactique non identifié ", Pratiques, 97-98, p. 59-103.

- (2001) : " Lecture cursive et lecture analytique. Du goût et du plaisir de lire ", Pratiques, 109-110, p. 179-205.

- (2004) : « Pour une lecture polyphonique. Assia Djebar : langage tangage, langage, tatouage », Pratiques, 123-124, p. 75-111.

- (2005) : «Expérience de lecture et expérience esthétique : du plaisir et de l'émotion », in :

V. Jouve (éd.), L'expérience de lecture, Paris, Éd. L'Improviste, p. 425-468.

- (2011) : “ “Il n'y a jamais que des contextes”. Les communautés interprétatives de Stanley Fish », Pratiques, 151-152, p. 49-72.

Milcent-Lawson, S. (2013) : «Poétique du défigement chez Giono et Beckett », Pratiques, 159-160, p. 129-146.

MitTERRAND, H. (1986) : Émile Zola, Carnets d'enquête. Une ethnographie inédite de la France, Paris, Plon (coll. « Terre humaine »).

- (1992) : « Les obsédés de l'objectif. L'enseignement du français en question », Le Débat, 71, p. $164-172$.

MOIRAND, S. (1982) : Enseigner à communiquer en langue étrangère, Paris, Hachette.

MoIsAn, C. (1987) : Qu'est-ce que l'histoire littéraire?, Paris, PUF.

MOLINIER, G. \& CAHNÉ, P. (dirs) (1994) : Qu'est-ce que le style, Paris, PUF.

Molino, J. (1984) : « L'expérience d'I. A. Richards », Poétique, 59, Paris, Éd. Le Seuil, p. 347-367.

- (1989) : « Interpréter », in : C. Reichler (éd.), L'interprétation des textes, Paris, Éd. de Minuit, p. 9-52.

Monballin, M. \& Legros, G. (1994) : « CEuvres romanesques et théâtrales en fin de secondaire : un singulier pluriel », Enjeux, 32, p. 7-22.

MonTE, M. (2012) : « Théories linguistiques et littéraires et ateliers d'écriture », Pratiques, 155-156, p. 205-222.

MounIN, G. (1976) : Linguistique et traduction, Bruxelles, Dessart/Mardaga.

NATUReL, M. (1995) : Pour la littérature, de l'extrait à l'œuvre, Paris, Clé internationale.

NEVEU, F. (2005) : «L'idiolecte, entre linguistique et herméneutique », Cahiers de praxématique, 44, p. 25-49.

NoËL-Gaudreault, M. (dir.) (1997) : Didactique de la littérature. Bilan et perspectives, Québec, Éd. Nuit Blanche (coll. « Les Cahiers du Centre de recherches en littérature québecoise »).

NonNON, E. (1992) : «Fonctions de l'aide et du questionnement de l'enseignant dans la lecture et la compréhension de textes ", Recherches, 17, p. 97-132. 
- (1998) : «Quelle transposition des théories du texte en formation des enseignants ? », Pratiques, 97-98, p. 153-170.

OlLIER, C. (1975) : « Gestes de lecture », Pratiques, 7-8, p. 21-24.

OLSON, D. R. (1998) : L'univers de l'écrit.Comment la culture écrite donne forme à la pensée, Paris, Retz.

ORIOL-BOYER, C. (dir.) (1988) : La réécriture, Grenoble, Éd. Atelier du texte/Ceditel.

ORIOL-BOYER, C. \& BILOUS, D. (dirs) (2013) : Ateliers d'écriture littéraire, Paris, Hermann.

PAGÉ, M. (1985) : « Lecture et interaction, lecture/texte, contribution à l'élaboration d'un modèle interactionniste de la lecture », in : Didactique de la lecture au secondaire, Montréal, Éd. Ville Marie.

PAGES, A. (2000) : «L'espace littéraire du naturalisme », Pratiques, 107-108, p. 89-114.

PASTIAUX-ThiRIAT, G. (1997) : « La recherche en didactique des textes littéraires dans la banque de données DAF/DAFTEL », in : M. Noël-Gaudreault (dir.), Didactique de la littérature. Bilan et Perspectives, Québec, Éd. Nuit Blanche (coll. « Les Cahiers du Centre de recherches en littérature québecoise »), p. 233-249.

PAVEAU, M.-A. (2010) : «Interdiscours et intertexte. Généalogie scientifique d'une paire de faux jumeaux », in : D. Ablali \& M. Kastberg Sjöblom (éds), Linguistique et littérature. Cluny, 40 ans après, actes du colloque de Besançon, Besançon, Presses universitaires de Franche-comté (coll. « Linguistique et sémiotique »), p. 93-105.

- (2011) : «L'analyse linguistique du texte littéraire. Une fausse évidence », Le Français aujourd'hui, 175, Paris, A. Colin, p. 83-94.

- (2013a) : «Genre de discours et technologie discursive.Tweet, twittécriture et twittérature », Pratiques, 157-158, p. 7-30.

- (2013b) : «La mémoire discursive », in : Langage et morale. Une éthique des vertus discursives, Limoges, Lambert-Lucas, p. 171-204.

- (à paraitre) : "En naviguant en écrivant. Réflexions sur les textualités numériques ", in :J.-M. Adam (éd.), Faire texte. Frontières textuelles et opérations de textualisation, Besançon, PUFC, p. 367-385.

PAVEL, T. (1988) : L’Univers de la fiction, Paris, Éd. Le Seuil.

PenNaC, D. (1992) : Comme un roman, Paris, Gallimard.

Penloup, M.-C. (1999) : L'écriture extrascolaire des collégiens, Paris, ESF.

PERRIN, C. (1996) : « Du projet d'année à la séquence : l'étude du Colonel Chabert en $3^{\mathrm{e}}$ », Pratiques, 92, p. 83-106.

PERRIN, L. (2004) : « Polyphonie et autres formes d'hétérogénéité énonciative : Bakhtine, Bailly, Ducrot... », Pratiques, 123-124, p. 7-26.

- (2013) : «De l'analysibilité au défigement des expressions figées. La leçon de Giono dans Les Âmes fortes ", Pratiques, 159-160, p. 109-126.

Petitjean, A. (1982) : Pratiques d'écriture, Paris, Nathan-Cedic (coll. « Textes et non-textes »).

- (1984) : « Pratiquer le théâtre à l'école », Pratiques, 41, p. 3-35.

- (1984) : « Pastiche et parodie, enjeux théoriques et pédagogiques », Pratiques, 42, p. 3-33.

- (1987) : « Exercices : analyses et productions de textes descriptifs », Pratiques, 56, p. 89-100.

- (1988) : «Approches du conte philosophique à partir de l'exemple de Candide », Pratiques, 59, p. 73-106.

- (1990) : « Pour une didactique de la littérature », in : D.-G. Brassart et al. (dirs), Perspectives didactiques en français, actes du Colloque de Cerisy Didactique et pédagogie du français, recherches 
actuelles, Cresef (coll. « Didactique des textes »), p. 101-127.

- (1994) : «Écrire des récits d'aventure », Pratiques, 83, p. 79-124.

- (1998a) : « Le statut de la littérature dans les instructions officielles du secondaire

(1986-1994)», Enjeux, 43-44, p. 91-121.

- (1998b) : «Enseignement/apprentissage de l'écriture et transposition didactique », Pratiques,

97-98, p. 105-132.

- (1999) : «Valeurs, savoirs et textes dans les Instruction officielles du lycée », Pratiques, 101-102,

p. $117-138$.

- (2001a) : « Nouveaux programmes et recherches en didactique de la littérature », in : Recherches en didactique de la littérature, textes réunis par M.-J. Fourtanier, G. Langlade \& A. Rouxel, Rennes, PUR, p. 57-59.

— (2001b) : « La description scolaire au secondaire (collège) de 1960 à 1997 », Pratiques, 109/110, p. $125-164$.

- (2003a) : « Histoire de l'écriture d'invention au lycée », Pratiques, 117-118, p. 181-207.

- (2003b) : « Problématisation du personnage dramatique », Pratiques, 119-120, p. 67-90.

- (2005) : «Écriture d'invention au lycée et acquisition de savoir et de savoir faire », Pratiques, 127-128, p. 75-96.

- (2009) : "Genres littéraires et descriptions linguistiques ", in : C. Hudelot \& C. Jacquet-Pfau (éds), Sciences du langage et demandes sociales, actes du colloque de l'Association des sciences du Langage, Limoges, Lambert-Lucas, p. 101-113.

- (2010) : «Linguistique et littérature : le style en question », in : D. Ablali \& M. Kastberg Sjöblom (éds), Linguistique et littérature. Cluny, 40 ans après, actes du colloque de Besançon, Besançon, PUFC (coll. « Linguistique et sémiotique »), p. 239-248.

- (2011) : « Pour une biographie sociologique : l'exemple de Bernard-Marie Koltès », in :

A. Petitjean, Bernard-Marie Koltès. Textes et contextes, Metz, Université Paul Verlaine-Metz (coll. « Recherches textuelles »), p. 155-168.

Petijean, A. \& FAVART, F. (2012) : « Faits de langue et effets de voix populaires dans les fictions romanesques ", in : actes du colloque international de Dijon Quand les genres de discours provoquent la grammaire... et réciproquement, textes réunis par C. Despierres \& M. Krazem, Limoges, LambertLucas, p. 77-87.

Petitjean, A. \& Halté, J.-F. (1974) : « Pour une théorie de l'idéologie d'un manuel scolaire, Le Lagarde et Michard : Le cas Diderot », Pratiques, 1-2, p. 43-64.

Petitjean, A, Halté, J.-F. \& Michel, R. (1974) : « Essai d'analyse structurale de Chat noir d'E.A. Poe, pour une application pédagogique », Pratiques, 1-2, p. 5-28.

Petitjean, A. \& Privat, J.-M. (dirs) (1998) : Histoire de l'enseignement du français et textes officiels, Metz, université Paul Verlaine-Metz (coll. « Didactique des textes »).

- (2007) : Les voix du peuple et leurs fictions, Metz, université Paul Verlaine-Metz (coll. «Recherches textuelles»).

Petitjean, A. \& Rabatel, A. (2007) : « Le style en question », Pratiques, 135-136, p. 3-14.

PetitjeAn, A.-M. (2013) : La littérature sur le métier. Études comparées des pratiques créatives d'écriture littéraire dans les universités de France, aux États-Unis et au Québec, thèse Nouveau Régime, université de Cergy-Pontoise.

Peytard, J. (1975) : « Le personnage dominant dans Le lieutenant Kije de I. Tynianov », Pratiques, 5, p. 85-94.

- (1982) : «Sémiotique du texte littéraire et didactique du FLE », Études de linguistique appliquée, 45, Didier Érudition, p. 91-103. 
- (1988) : «Des usages de la littérature en classelangue », Le Français dans le monde, numéro spécial, « Littérature et enseignement », p. 8-17.

PhiLIPPE, G. (2000) : « Les divergences énonciatives dans les récits de fiction », Langue française, 128 , p. 30-51.

- (2005) : «Traitement stylistique et idiolectal des singularités langagières », Cahiers de praxématique, 44 , p. 77-91.

- (2009) : «La langue littéraire et la langue parlée », in : G. Philippe \& J. Piat (dirs), La langue littéraire Une histoire de la prose en France de Gustave Flaubert à Claude Simon, Paris, Fayard, p. 57-89.

PhILIPPE, G. \& PIAT, J. (dirs) (2009) : La langue littéraire. Une histoire de la prose en France de Gustave Flaubert à Claude Simon, Paris, Fayard.

PICARD, M. (1986) : La lecture comme jeu, Paris, Éd. de Minuit.

PlANE, S. (2000) : « Éléments pour un usage didactique du traitement de texte. Écrire, réécrire et réviser sur ordinateur ", Pratiques, 105-106, p. 159-181.

- (2001) : «La réception des nouvelles approches de l'écriture d'invention au lycée : le difficile passage de l'écriture du lycéen à l'écriture de l'auteur ", Lidil, 23, p. 67-82.

Popovic, P. (2011) : « La sociocritique, histoire, concepts, voies d'avenir », Pratiques, 151-152, p. 7-38.

PORCHER, L. (1988) : « Programme, progrès, progression, projets dans enseignement/apprentissage d'une culture étrangère ", Études de linguistique appliquée, 69.

PORCHER, L. \& ZARATE, G. (2001) : Éducation et communication interculturelle, Paris, PUF.

Pouivet, R. (1999) : L'Ontologie de l'œuvre d'art. Une introduction, Nîmes, J. Chambon.

Poulain, M. (dir.) (1988) : Pour une sociologie de la lecture. Lecture et lecteur dans la France contemporaine, Paris, Editions du Cercle de la Librairie.

Privat, J.-M. (1993) : «L'institution des lecteurs », Pratiques, 80, p. 7-34.

- (1995) : «Culture littéraire et métalangages culturels », in : Les Métalangages de la classe de français, textes réunis et présentés par R. Bouchard et J.-C. Meyer, actes du $6^{\mathrm{e}}$ colloque de la DFLM, p. 17-24.

- (1997) : «Introduction à la lecture ethno-critique en $3^{\mathrm{e}}$ », Pratiques, 95, p. 53-95.

PRIVAT, J.-M. \& ReUter, Y. (dirs) (1991) : Lectures et médiations culturelles, actes du colloque de Villeurbanne, Lyon, PUL.

PRIVAT, J.-M. \& VinSON, M.-C. (1996) : « La fiche de lecture ou la bureaucratisation d'une technique d'animation culturelle », Pratiques, 90, p. 83-94.

- (1999) : «Le statut du livre et du lecteur dans les instructions officielles du primaire et du secondaire ", Pratiques, 101-102, p. 105-115.

- (2008) : « Façons de lire, façons de faire », Pratiques, 137-38, p. 199-216.

RABATEL, A. (1998) : Une histoire du point de vue, Metz, université Paul Verlaine-Metz (coll. « Recherches textuelles »).

- (2003) : « Le problème du point de vue dans le texte de théâtre », Pratiques, 119-120, p. 7-33.

- (2004a) : Argumenter en racontant. Une introduction à la lecture et à l'écriture littéraires, Bruxelles, De Boeck.

- (2004b) : «Effacements argumentatifs et effets argumentatifs indirects dans l'incipit du Mort qu'il faut de Semprun ", Semen, 17, p. 112-132.

- (2005) : « La construction inférentielle des valeurs. Propositions pour une pragmatique énonciative des textes littéraires », Cahiers de narratologie, 12, « Récit et éthique », p. 1-18. 
- (2006) : « La lecture comme activité de construction », Lidil, 33, p. 55-77.

- (2007) : «La dialectique du singulier et du social dans les processus de singularisation : style(s), idiolecte, ethos ", Pratiques, 135-136, p. 15-34.

- (2008a) : Homo narrans, t. 1, Les points de vue et la logique de la narration; t. 2, Dialogisme et polyphonie dans le récit, Limoges, Lambert-Lucas.

- (2008b) : La construction textuelle du point de vue, Lausanne, Delachaux et Niestlé.

RASTIER, F. (1972) : « Un concept dans le discours des études littéraires », Littérature, 7, p. 87-101.

- (1989) : Sens et textualité, Paris, Hachette.

- (2013) : Apprendre pour transmettre. L'éducation contre l'idéologie managériale, Paris, PUF

(coll. « Souffrance et théorie »).

REICHLER, C. (1992) : « Une nouvelle approche en histoire littéraire », Le Français aujourd'hui, 97, p. 39-44.

ReUTER, Y. (1981) : «Le champ littéraire : textes et institutions », Pratiques, 32, p. 5-29.

- (1982) : «Les pratiques thématiques dans le secondaire », in : J.-F. Halté \& A. Petitjean (dirs), Pour un nouvel enseignement du français, Bruxelles, De Boeck/Duculot, p. 67-77.

- (1987) : « Descriptions de femmes (Un atelier de descripture) », Pratiques, 55, p. 47-60.

- (1990) : «Définir les biens littéraires », Pratiques, 67, p. 5-14.

- (1992) : «Comprendre, interpréter, expliquer des textes en situation scolaire. À propos d'Angèle

", Pratiques, 76, p. 7-25.

- (1994) : «La notion de scène : construction théorique et intérêts didactiques », Pratiques, 81, p. 5-26.

- (1995) : «La lecture littéraire : éléments de définition », Le Français aujourd'hui, 112, p. 65-71.

- (1996a) : «Éléments de réflexion sur la place et les fonctions de la littérature dans la didactique du français à l'école primaire ", Repères, 16, p. 7-25.

- (1996b) : Enseigner et apprendre à écrire, Paris, ESF.

- (1999) : «L'enseignement-apprentissage de la littérature en questions », Enjeux, 43-44,

p. 191-203.

- (2000) : « La prise en compte de l'inscription sociale dans l'enseignement-apprentissage de la littérature ", Pratiques, 107-108, p. 55-69.

- (2001) : Comprendre et interpréter le littéraire à l'école, Paris, INRP.

REUTER, Y. \& LAHANIER-REUTER, D. (2007) : « L'analyse de la discipline : quelques problèmes pour la recherche en didactique ", in : E. Falardeau, C. Fisher, C. Simard, \& N. Sorin, (dirs), La didactique du français. Les voies actuelles, Québec, Presses universitaires de Laval, p. 27-42.

ReUTER, Y. (éd.) (2007a) : « Disciplines scolaires », in : Y. Reuter, C. Cohen-Azria, B. Daunay, I. Delcambre \& D. Lahanier-Reuter, Dictionnaire des concepts fondamentaux des didactiques, Bruxelles, De Boeck, p. 85-89.

- (2007b) : «Forme scolaire », in : Y. Reuter, C. Cohen-Azria, B. Daunay, I. Delcambre \&

D. Lahanier-Reuter, Dictionnaire des concepts fondamentaux des didactiques, Bruxelles, De Boeck, p. 111-115.

RicARdou, J. (1978) : « Écrire en classe », Pratiques, 20, p. 23-70.

RICHARDS, Ivor A. (1929): Pratical Criticism, Londres.

RICGUR, P. (1985) : « Mimésis III », in : Temps et récit, t. 1, Paris, Éd. Le Seuil.

- (1986) : Du texte à l'action. Essais d'herméneutique II, PAris, Éd. Le Seuil (coll. « Points Essais »).

RIGAT, F. (2012) : Aspects linguistiques du texte expographique, Torino, Trauben. 
RIVENC, P. (1964) : « Deux méthodes audio-visuelles d'enseignement du français comme langue étrangère ", in : Recherches et Techniques nouvelles au service de l'enseignement des langues vivantes, Strasbourg, Conseil de l'Europe.

RoBINE, N. (1984) : Les jeunes travailleurs et la lecture, Paris, Documentation Française.

RORTY, R. (1992) : «Le parcours du pragmatiste », in : U. Eco, Interprétation et surinterprétation, Paris, PUF, p. 81-99.

ROsIER, J.-M. (1998) : « Réflexions didactiques sur la littérature francophone de Belgique », Textyles, 15, «L'institution littéraire », p. 166-174.

ROUXEL, A. (1996) : Enseigner la lecture littéraire, Rennes, PUR.

- (2001) :» Lecture cursive/lecture analytique : construire la complémentarité », in : Recherches en didactique de la littérature, textes réunis par M.-J. Fourtanier, G. Langlade \& A. Rouxel, Rennes, PUR, p. 75-79.

- (2005) : «Identité disciplinaire : éclatement ou cohérence », in : A. Brillant-Annequin \& J.-F. Massol (dirs), Le pari de la littérature. Quelles littératures de l'école au lycée ?, Grenoble, CRDP de Grenoble, p. 22-28.

Rouxel, A., \& LANGLADE, G. (dirs) (2004) : Le sujet lecteur. Lecture subjective et enseignement de la littérature, Rennes, PUR.

RUDLER, G. (1902) : L'Explication française : principes et applications, Paris, A. Colin.

SAGNET, H. (2009) : «Internet, nouvel espace de légitimation adolescente des œuvres ? L'exemple des fanfictions sur Fascination », Lecture jeune, 129, « Les classiques de la littérature pour adolescents » (en ligne : http://www.lecturejeunesse.com).

SANGSUE, D. (2007) : La relation parodique, Paris, Corti.

SALLENAVE, D. (1995) : Lettres mortes, de l'enseignement des lettres en général et de la culture générale en particulier, Paris, Michalon.

SCARPA, M. (2000) : « Des avant-textes au roman : l'exemple du Ventre de Paris. Approche génétique et ethnocritique », Pratiques, 107-108, p. 71-88.

SCHAEFFER, J.-M. (1992) : L'Art de l'âge moderne. L'esthétique et la philosophie de l'art du XVIII siècle à nos jours, Paris, Gallimard.

- (1996) : Les Célibataires de l'art. Pour une esthétique sans mythes, Paris, Gallimard.

- (1999) : Pourquoi la fiction, Paris, Éd. Le Seuil.

- (2010) : « Le traitement cognitif de la narration », 215-232.

- (2011) : Petite écologie des études littéraires, Pourquoi et comment la littérature, Vincennes,

T. Marchaisse.

SCHMIDT, S.-J. (1973) : « La communication littéraire », in : Stratégies discursives, Lyon, PUL, p. 19-31.

Simone, R. (2012) : Pris dans la toile. L'esprit au temps du web, Paris, Gallimard.

SMITH, F. (1975) : Comment les enfants apprennent à lire, Paris, Retz.

- (1986) : Devenir lecteur, Paris, A. Colin/Bourrelier.

SchmitT, Michel, P. (2006) : « École et dégoût littéraire », Lidill, 33, p. 161-170.

SCHNEDECKER, C. (1990) : « Un genre descriptif : le portrait », Pratiques, 66, p. 59-106. 
SCHNEUWLY, B. (2007) : « Le "Français" une discipline scolaire autonome, ouverte et articulée », in : E. Falardeau, C. Fisher, C. Simard \& N. Sorin (dirs), La didactique du français. Les voies actuelles, Québec, Presses universitaires de Laval, p. 9-26.

SEARLE, J. (1982 [1975]) : « Le statut logique du discours de la fiction », in : Sens et Expression, trad. de l'américain, Paris, Éd. de Minuit, p. 101-119.

- (1985 [1983]) : L'intentionalité, trad. de l'américain, Paris, Éd. de Minuit.

SÉrTÉ, Y. (2000) : « La théorie littéraire questionnée par l'histoire », Textuel, 37, p. 181-199.

SÉOUD, A. (1997) : Pour une didactique de la littérature, Paris, Crédif/Hatier/Didier (coll. « LAL »).

SHUSTERMAN, R. (1999 [1994]) : La Fin de l'expérience esthétique, trad. de l'américain, Pau, PUP (coll. «Quad»).

SITRI, F. (2003) : « La diffusion de la "typologie des textes" dans les manuels scolaires : formes et enjeux », in : R. Amossy \& D. Maingueneau (dirs), L'analyse du discours dans les études littéraires, colloque de Cerisy, Toulouse, Presses universitaires du Mirail, p. 389-403.

Schмiтt, M. P. (20006) : «École et dégoût littéraire », Lidil, 33, p. 161-170.

SPERBER, D. \& WiLSON, D. (1989 [1986]) : La pertinence, trad. de l'anglais, Paris, Éd. de Minuit.

SPRENGer-Charolles, L. (1983) : " Analyse d'un dialogue didactique : l'explication de texte ", Pratiques, 40, p. 51-76.

SPRENGER-Charolles, L \& ColÉ, P. (2013) : Lecture et dyslexie. Approche cognitive, $2^{\mathrm{e}}$ éd., Paris, Dunod. SULEIMAN, S. R. (1983) : Le roman à thèse, Paris, PUF.

TAUVERON, C. (2001) : « Littérature de jeunesse ou nouvelle jeunesse pour la littérature et son enseignement? ", in : Perspectives actuelles de l'enseignement du français, actes du séminaire national organisé par la direction de l'Enseignement scolaire, ministère de l'Enseignement scolaire, p. 193-200.

- (2002) : Lire la littérature à l'école, Paris, Hatier.

- (2009) : "L'interprétation à l'école : des sources théoriques à une modélisation didactique ", in : M. Butlen \& V. Houdart-Merot (dirs), Interpréter et transmettre la littérature aujourd'hui, Encrage, université de Cergy-Ponyoise, p. 53-75.

TAUVERon, A.-M. (2000) : « Le réseau intertextuel de L'œuvre de Zola. Une démarche de lecture/ écriture ", Pratiques, 107-108, p. 115-144.

THÉRIEN, M. (1997) : « De la définition du littéraire et des œuvres à proposer aux jeunes », in : M. Noêl-Gaudrault (dir.), Didactique de la littérature. Bilan et perspective, Québec, Éd. Nuit blanche.

THÉRIEN, M. \& ForTIER, G. (dirs) (1985) : Didactique de la lecture au secondaire, Québec, Éd. VilleMarie.

THIBAUDEAU, J. (1971) : « Notes sur quelques manuels de littérature française », Littérature/Science/ idéologie, 1, p. 6-9.

- «École/Langue/Texte (entretien) », Pratiques, 3/4, p. 61-92.

ToDorov, T. (1965) : Théorie de la litérature (textes des formalistes russes), Paris, Éd. Le Seuil.

- (1978) : «La notion de littérature », in : Les genres du discours, Paris, Éd. Le Seuil.

- (2007) : La littérature en péril, Paris, Flammarion.

VAUDREY-LUIGI, S. (2011) : " De la signature stylistique à la reconnaissance d'un style d'auteur », Le Français aujourd'hui, 175, p. 37-45. 
VAugEoIs, D. (dir.) (2005) : « L'écrit sur l'art : un genre littéraire? », Figure de l'Art, 9, Pau, Publication de l'université de Pau.

VECK, B. (dir.) (1988) : Production de sens. Lire/Ecrire en classe de seconde, Paris, INRP (coll. « Rapports de recherches en classe de seconde $")$.

- (dir.) (1990) : Enseignement du français dans le second cycle. Trois savoirs pour une discipline, Paris, INRP.

- (dir.) (1994) : La culture littéraire au lycée : des humanités aux méthodes, Paris, INRDP.

VECK, B., ROBERT-LAZĖs, \& C., ROBERT, M. (1996) : Français au baccalauréat. Observatoire des listes d'oral. Session 1996, Paris, INRDP.

VERNET, C. (1995) : « La littérature policière de jeunesse : caractéristiques des genres, propositions didactiques ", Pratiques, 88, p. 81-122.

ViALA, A. (1989) : « Pour une périodisation du champ littéraire », in : C. Clément (dir.), Histoire littéraire, théories, méthodes, pratiques, Québec, Les Presses universitaires de Laval, p. 93-124.

- (2000) : « Théories littéraires, théorie du texte et histoire », Textuel, 37, p. 201-223.

- (2001) : « Les nouveaux programmes de lycée : éléments pour un débat », in : Recherches en didactique de la littérature. Rencontres de Rennes, textes réunis par M-J. Fourtanier, G. Langlade \& A. Rouxel, Rennnes, PUR, p. 47-55.

- (2004) : « Le littéraire, son enseignement et le social », Le Français aujourd'hui, 145, p. 5-14.

Viala, A., Chervel, A., SARrazin, B. \& Rohou, J., (1990) : « Naissance et évolution de la dissertation ", Pratiques, 68, p. 107-118.

VIGNE, E. (2008) : Le Livre et l'Éditeur, Paris, Klincksieck.

VINCENT, G. (dir) (1994) : L'Éducation prisonnière de la forme scolaire ? Scolarisation et socialisation dans les sociétés industrielles, Lyon, PUL.

Vincent-Buffault, A. (1986) : Histoire des larmes. XVIII - XIX $X^{e}$ siècles, Paris, Petite Bibliothèque Payot.

VINSON, M.-C. (1987) : « Descriptions et point de vue : un travail de lecture/écriture au collège », Pratiques, 56, p. 89-99.

- (2006) : «L'index, une technologie de l'intellect », Pratiques, 131-132, p. 199- 216.

VinSON, M.-C. \& LELIÈVRE-PoRTALIER, D. (1993) : « La bouquinerie au collège : un nouveau marché de lectures ", Pratiques, 80, p. 35-53.

Vouilloux, B. (1992) : « Le Texte et l'image : où commence et comment finit une interdiscipline? », Littérature, 87, p. 95-98.

- (1998) : « Pour une théorie descriptiviste du style », Poétique, 114, Paris, Éd. Le Seuil, p. 233-254.

- (2004) : «Critique : le genre et l'espèce », in : L'œuvre en souffrance, entre poétique et esthétique, Paris, Belin.

- (2005) : « Les prédicats stylistiques », in : J.-M. Gouvard (dir.), De la langue au style, Lyon, PUL

(coll. « Texte et langue »), p. 315-355.

- (2006) : « Le jeu des valeurs. Au-delà de l'esthétique », Revue des Sciences Humaines, 283, p. 43-62.

VouRzAY, M.-H. (1998) : « Jalons pour une histoire de la description scolaire : deux "modèles didactiques" de la rédaction/description pour le premier cycle du secondaire ", Pratiques, 99, p. 27-69.

_ (2001) : « La description scolaire au secondaire (collège) de 1900 à 1960 », Pratiques, 109-110, p. 67-123. 
VultuR, I. (2005) : « La réception de la "Recherche" : une question de genre ? », Poétique, 142, Paris, Éd. Le Seuil, p. 239-254.

WeInLANd, C. \& Puygrenier-Renault, L. (1997) : L'enseignement du français au collège, BertrandLacoste.

WIEVIORKA, M. (2013) : L'impératif numérique ou la nouvelle ère des sciences humaines et sociales ?, Paris, CNRS Éd.

\section{Revues citées}

Actes de la recherche en sciences sociales, 123 (1998) : «Genèse de la croyance Littéraire », Paris, Éd. Le Seuil.

Cahiers de praxématique, 44 (2005), «L’idiolecte. Du singulier dans le langage », Montpellier III.

Communications, 30 (1979) : «Logique et conversation », Paris, Éd. Le Seuil.

Cultures Études, 5. (2009) : « Les pratiques culturelles des Français à l'ère numérique », Paris, DEPS.

Enjeux, 32 (1994) : «Corpus et lectures littéraires », Namur, Faculté Notre-Dame de la Paix.

Enjeux, 43-44 (1999) : «Littérature : les programmes francophones », Namur, Faculté Notre-Dame de la Paix.

Enjeux, 49 (2000) : « Enseignement de la littérature et compétence de communication », Namur, Faculté Notre-Dame de la Paix.

Enjeux, 51/52 (2001) : « Recherches en didactique de la littérature », Namur, Faculté Notre-Dame de la Paix.

Enjeux, 55 (2002) : « Former des enseignants de lecture », Namur, Faculté Notre-Dame de la Paix.

Enjeux, 57 (2003) : «Littérature et écriture d'invention », Namur, Faculté Notre-Dame de la Paix.

Esprit, 12. (1974) : « Lecture 1 : l'espace du texte », Paris.

Études de linguistique appliquée, 45 (1982) : «Littérature à enseigner », Paris, Didier Érudition.

Études de linguistique appliquée, 52 (1983) : «Littérature de jeunesse », Paris, Didier Érudition.

Études de linguistique appliquée, 118 (2000) : « La crise du français », Paris, Didier Érudition.

Formules, 5 (2001) : « Pastiches, collages et autres réécritures », Paris, Noésie.

Histoire de l'éducation, 94 (2002) : numéro spécial, « Évaluer, sélectionner, certifier. $\mathrm{XVI}^{\mathrm{e}}-\mathrm{XX}^{\mathrm{e}}$ siècles ", Paris, INRP.

L'Année sociologique, 22 (1971), Paris, PUF.

La Nouvelle Critique (1969) : numéro spécial, « Linguistique et Littérature », colloque de Cluny.

Langages, 31 (1973) : « Sémiotiques textuelles », Paris, Larousse.

Langue française, 7 (1970) : «La description linguistique des textes littéraires », Paris, Larousse.

Langue française, 121 (1999) : " Phrase, Texte, discours », Paris, Larousse.

Le Débat, 71(1992 : « Dossier Langue, Littérature, Humanités », Paris, Gallimard.

Le Débat, 110 (2000) : « 20 ans », Paris, Gallimard.

Le Débat, 170 (2012) : « Le livre, le numérique », Paris, Gallimard. 
Le Français aujourd'hui, 49 (1980) : «L'histoire dans la classe de français », Paris, A. Colin.

Le Français aujourd'hui, 72 (1985) : « Histoire littéraire 1 », Paris, A. Colin.

Le Français aujourd'hui, 73 (1986) : « Histoire littéraire 2 », Paris, A. Colin.

Le Français aujourd'hui, 90 (1990) : «Lecture(s) méthodiques », Paris, A. Colin.

Le Français aujourd'hui, 93 (1991) : « Concevoir l'écrit », Paris, A. Colin.

Le Français aujourd'hui, 97 (1992) : «Le groupement de textes », Paris, A. Colin.

Le Français aujourd'hui, 112 (1995) : « Lecteurs, lectures », Paris, A. Colin.

Le Français aujourd'hui, 127 (1999) : « Ecritures créatives », Paris, A. Colin.

Le Français aujourd'hui, Hors série (1999) : « Lecteurs, Littérature, Enseignement », Paris, A. Colin.

Le Français aujourd'hui, 128 (1999) : «L'énonciation : questions de discours », Paris, A. Colin.

Le Français aujourd'hui, 137 (2002) : «L'attention aux textes », Paris, A. Colin.

Le Français aujourd'hui, 141 (2003) : « Enseigner la langue de l'école au lycée », Paris, A. Colin.

Le Français aujourd'hui, 145 (2004) : « Le littéraire et le social », Paris, A. Colin.

Le Français aujourd'hui, 149 (2005) : « La Littérature de jeunesse : repères, enjeux et pratiques », Paris, A. Colin.

Le Français aujourd'hui, 153 (2006) : « Enseigner l'écriture littéraire », Paris, A. Colin.

Le Français aujourd'hui, 155 (2006) : « Lecture des textes fondateurs », Paris, A. Colin.

Le Français aujourd'hui, 168 (2010) : «Continuités et ruptures dans l'enseignement de la littérature, Paris, A. Colin.

Le Français aujourd'hui, 172 (2011) : » Corpus littéraires en question », Paris, A. Colin.

Le Français aujourd'hui, 175 (2011) : " Littérature et linguistique : dialogue ou coexistence ?», Paris, A. Colin.

Le Français aujourd'hui, 178 (2012) : « L'enseignement des lettres et le numérique », Paris, A. Colin.

Le Français aujourd'hui, 182 (2013) : « Histoire des arts : de la notion à la discipline », Paris, A.

Colin.

Le Français dans le monde (1988) : numéro spécial, « Littérature et enseignement. La perspective du lecteur ", Paris, Larousse.

Les Cahiers du CRELEF, 25 (1987) : «Questionnement pédagogique et texte littéraire », Besançon, université de Besançon.

Lidil, 23 (2001) : « Les nouveaux écrits à l'école : nouveaux programmes, nouvelles pratiques, nouveaux savoirs », Lidilem, université de Grenoble.

Lidil, 33 (2006) : «La réception des textes littéraires. Une didactique en construction », Lidilem, université de Grenoble.

Littérature, 7 (1972) : « Le discours de l'école sur les textes », Paris, Larousse.

Littérature, 42 (1980) : «L'institution littéraire I », Paris, Larousse.

Littérature, 44 (1981) : « L'institution littéraire II » », Paris, Larousse.

Littérature, 87 : « La moire et l'image », Paris, Larousse. 1, 
Littérature/Science/idéologie, 1, Paris.

Modèles linguistiques, tome xxxI, vol 60 (2009) : « L'écriture mimétique I », Paris, Éd. Les Dauphins.

Modèles linguistiques, tome XXXI, vol. 61 (2010) : « L'écriture mimétique II », Paris, Éd. Les

Dauphins.

Poétique, 16 (1973) : « Le discours réaliste », Paris, Éd. Le Seuil.

Poétique, 30 (1977) : « Enseignements », Paris, Éd. Le Seuil.

Poétique, 59 (1984) : Paris, Éd. Le Seuil.

Poétique, 114 (1998) : Paris, Éd. Le Seuil.

Poétique, 142 (2005) : Paris, Éd. Le Seuil.

Pratiques, 1/2 (1974), Metz, Cresef.

Pratiques, $3 / 4$ (1974), Metz, Cresef.

Pratiques, 5 (1975), Metz, Cresef.

Pratiques, 7/8 (1975) : « Lire », Metz, Cresef.

Pratiques, 10, (1976) : « Enjeux des textes », Metz, Cresef

Pratiques, 11/12 (1976) : " Récit 1 », Metz, Cresef.

Pratiques, 13, (1976) : « Textes - linguitique », Metz, Cresef.

Pratiques, 20 (1978) : « Les mots ont la parole », Metz, Cresef.

Pratiques, 21 (1978) : « Proésies », Metz, Cresef.

Pratiques, 22-23 (1979) : « Lectures suivies », Metz, Cresef.

Pratiques, 27 (1980) : «L'écrivain aujourd'hui », Metz, Cresef.

Pratiques (1980) : numéro spécial, « Pour un nouvel enseignement du français », colloque de Cerisy, Metz, Cresef.

Pratiques, 29 (1981) : «La composition française », Metz, Cresef.

Pratiques, 32 (1981) : « La littérature et ses institutions », Metz, Cresef.

Pratiques, 35 (1982) : « La lecture », Metz, Cresef.

Pratiques, 36 (1982) : « Travailler en projet », Metz, Cresef.

Pratiques, 38 (1983) : « Enseigner la littérature », Metz, Cresef.

Pratiques, 39 (1983) : « Le bricolage poétique », Metz, Cresef.

Pratiques, 40 (1983) : « La Communication », Metz, Cresef.

Pratiques, 41 (1984) : « L'écriture théâtrale », Metz, Cresef.

Pratiques, 42 (1984) : « L'écritue-imitation », Metz, Cresef.

Pratiques, 45 (1985) : « Les récits de vie », Metz, Cresef.

Pratiques, 47 (1985) : « Littérature de jeunesse », Metz, Cresef.

Pratiques, 50 (1986) : « Les paralittératures », Metz, Cresef.

Pratiques, 53 (1987) : « Pédagogie différenciée », Metz, Cresef. 
Pratiques, 54 (1987) : « Les mauvais genres », Metz, Cresef.

Pratiques, 55 (1987) : « Les textes descriptifs », Metz, Cresef.

Pratiques, 56 (1987) : « Les types de textes », Metz, Cresef.

Pratiques, 57 (1988) : «L'organisation des textes », Metz, Cresef.

Pratiques, 59 (1988) : « Les genres du récit », Metz, Cresef.

Pratiques, 60 (1997) : « Le personnage », Metz, Cresef.

Pratiques, 61 (1989) : « Ateliers d'écriture », Metz, Cresef.

Pratiques, 62 (1989) : «Classer les textes », Metz, Cresef.

Pratiques, 63 (1989) : «L'innovation pédagogique », Metz, Cresef.

Pratiques, 64 (1989) : « Paroles de personnages », Metz, Cresef.

Pratiques, 65 (1989) : « Dialogues de romans », Metz, Cresef.

Pratiques, 66 (1990) : « Didactique des genres », Metz, Cresef.

Pratiques, 67 (1990) : « Pratiques des textes littéraires », Metz, Cresef.

Pratiques, 68 (1990) : « La dissertation », Metz, Cresef.

Pratiques, 69 (1991) : « Textes et Histoire », Metz, Cresef.

Pratiques, 74 (1992) : « Pratiques textuelles théâtrales », Metz, Cresef.

Pratiques, 76 (1992) : «L'interprétation des textes », Metz, Cresef.

Pratiques, 77 (1993) : «Écriture et langue », Metz, Cresef.

Pratiques, 78 (1993) : « Didactique du récit », Metz, Cresef.

Pratiques, 80 (1993) : «Pratiques de lecteurs », Metz, Cresef.

Pratiques, 81 (1994) : " Scènes romanesques », Metz, Cresef.

Pratiques, 83 (1994) : «Écrire des récits », Metz, Cresef.

Pratiques, 85 (1995) : «Cohésion textuelle », Metz, Cresef.

Pratiques, 86 (1995) : « Lecture/écriture », Metz, Cresef.

Pratiques, 88 (1995) : « La littérature de jeunesse au collège », Metz, Cresef.

Pratiques, 89 (1996) : « Ecriture et créativité », Metz, Cresef.

Pratiques, 90 (1996) : « Des méthodes en français », Metz, Cresef.

Pratiques, 91 (1996) : « Les fables de La Fontaine », Metz, Cresef.

Pratiques, 92 (1996) : « Séquences didactiques », Metz, Cresef.

Pratiques, 93 (1997) : « Enseigner la poésie moderne », Metz, Cresef.

Pratiques, 95 (1996) : « La lecture littéraire en $3^{\text {ème} /} 2^{\mathrm{de}}$ », Metz, Cresef.

Pratiques, 97-98 (1998) : « La transposition didactique en français », Metz, Cresef.

Pratiques, 99 (1998) : « La description », Metz, Cresef.

Pratiques, 101-102 : «Textes officiels et enseignement du français », Metz, Cresef.

Pratiques, 105-106 : (2000) : « La réécriture », Metz, Cresef. 
Pratiques, 107/108 (2000) : « Les nouveaux programmes du lycée », Metz, Cresef.

Pratiques, 109/110 (2001) : « Histoire de la description scolaire », Metz, Cresef.

Pratiques, 113-114, 2002 : « Images du scripteur et rapports à l'écriture » Metz, Cresef,

Pratiques, 117/118 (2003) : « Textes et valeurs », Metz, Cresef.

Pratiques, 119/120 (2003) : « Les écritures théâtrales », Metz, Cresef.

Pratiques, 123-124 (2004) : « Polyphonie », Metz, Cresef.

Pratiques, 127/128 (2005) : «L'écriture d'invention », Metz, Cresef.

Pratiques, 131-132 (2006) : « La littératie. Autour de Jack Goody », Metz, Cresef.

Pratiques, 133-134 (2007) : « Récits, et disciplines scolaires », Metz, Cresef.

Pratiques, 135-136 (2007) : «Questions de style », Metz, Cresef.

Pratiques, 137-138 (2008) : " La didactique du français. Hommage à Jean-François Halté », Metz, Cresef.

Pratiques, 143-144 (2009) : «Écrits de savoirs », Metz, Cresef.

Pratiques, 145-146 (2010) : « Didactique du français (1)», Metz, Cresef.

Pratiques, 149-150 (2011) : « Didactique du français (2) », Metz, Cresef,

Pratiques, 151-152 (2011) : « Anthropologie de la littérature », Metz, Cresef.

Pratiques, 155- 156 (2012) : « Lexique et écriture », Metz, Cresef.

Pratiques, 155- 156 (2012) : « Lexique et écriture », Metz, Cresef.

Pratiques, 157-158 (2013) : « Théories et pratiques des genres », Metz, Cresef.

Pratiques, 159-160 (2013) : «Le figement en débat », Metz, Cresef.

Questions de communication, 17 (2010) : « Les cultures des sciences en Europe », Nancy, PUN.

Questions de communication, 18 (2010) : « Les usagers des TIC », Nancy, PUN.

Questions de communication, 21 (2012) : « 10 ans déjà, 10 questions de communication », Nancy, PUN-Éditions universitaires de Lorraine.

Recherches, 17 (1992) : «Le Mal de lire : apprentissages ratés », Lille, AFEF.

Recherches, 30 (1999) : « Parler des textes », Lille, AFEF.

Recherches, 39 (2003) : « Écriture d'invention », Lille, AFEF.

Repères, 13 (1996) : « Lecture et écritures littéraires à l'école », Paris, INRP.

Repères, 19 (1999) : « Comprendre et interpréter les textes à l'école », Paris, INRP.

Repères, 34 (2006) : « L'écriture de soi et l'école, École Normale Supérieure de Lyon, Institut français de l'éducation.

Repères, 37, (2008) : « Pratiques effectives de la littérature à l'école et au collège », Paris, INRP.

Repères, 40, (2009) : «Écrire avec, sur, de la littérature », Paris, INRP.

Repères, 43, (2011) : «Parler et écrire sur les œuvres : une approche interdidactique des enseignements artistiques et culturels ", École Normale Supérieure de Lyon, Institut français de l'éducation. 
Repères, 46, (2012) : « Vingt ans de recherches en didactique du français (1990-2010). Quelques aspects des recherches dans les revues ", École Normale Supérieure de Lyon, Institut français de l'éducation.

Revue française de pédagogie, 75 (1983) : «Comprendre un texte », Paris, INRP.

Revue française de pédagogie, 113 (1995) : « Lecture-écriture », Paris, INRP.

Revue française de pédagogie, 142, (2003) : Paris, INRP.

Revue française de pédagogie, 159 (2007) : « Politiques et rhétoriques de l'“École juste” avant la Cinquième République », Paris, INRP.

Revue de métaphysique et de morale, XII, 1904, Paris.

Revue des Sciences Humaines, 283 (2006) : « La valeur », Presses universitaires du Septentrion.

Semen, 17 (2004) : «Argumentation et prise de position : pratiques discursives », université de Franche-Comté.

Scolies, 1 (1971), Paris.

Textuel, 20 (1987) : «Comment expliquer. L'explication de textes », Revue de l'UFR de Lettres, («Sciences des Textes et Documents »), Paris, Université Paris VII.

Textuel, 37 (2010) : « Où en est la théorie littéraire », Paris, Revue de l'UFR de Lettres («Sciences des Textes et Documents »), Paris 7, Paris VII.

Textyles, 15 (1998) : « L'Institution littéraire », Bruxelles, Le Cri Éd.

Verbum, (2010) : tome XxxII, 2 : «Linguistique textuelle : état des lieux », Nancy, PUN.

\section{RÉSUMÉS}

L'article a pour objectif de faire un bilan des recherches consacrées à la «lecture littéraire » au niveau du secondaire. Pour ce faire, la revue Pratiques a été privilgiée, sans négliger pour autant d'autres publications parues depuis quarante ans. Dans une première partie, il s'agit d'expliciter les raisons pour lesquelles l'enseignement de la littérature est une discipline qui connait un état de crise quasi structurel depuis sa création. Ce faisant, on procède à une brève rétrospective de l'enseignement de la littérature depuis qu'à la fin du XIx ${ }^{e}$ siècle il s'est progressivement substitué à la rhétorique. Il en ressort que l'état de crise de cet enseignement est permanent mais a connu diverses variations conjoncturelles. Dans une seconde partie, on examine les apports théoriques de différents champs de référence concernant les textes littéraires et leurs lectures ainsi que les réponses didactiques apportées aux problèmes que pose l'enseignement/apprentissage de la lecture des textes littéraires. Dans une troisième partie,on met en perspective la «lecture littéraire » tant par rapport à l'enseignement/apprentissage de l'écriture que par rapport à des problématiques (textes et histoire; textes et valeurs) qui la prolongent. Dans une quatrième partie, on donne un aperçu de l'état actuel des théories de référence ainsi que des orientations présentes des recherches en didactique de la littérature dont celles qui ont cours au sein de la revue Pratiques. Dans la conclusion, on interroge la notion de «compétence » afin de mesurer sa pertinence pour l'enseignement/apprentissage de la « lecture littéraire ».

The purpose of this article is to asses the specifie researches on the teaching of the "literary reading" at the secondary level. The journal Pratiques has been chosen to analyse these researches for the last 40 years, however other publications edited by the same time have not been discriminated. In the first part of the article, we try to explain the reasons why the teaching 
of literature is a subject which has faced a quasi-structural crisis since its introduction. In so doing, we propose to make a retrospective review of the teaching of literature since it has gradually replaced rhetoric from the end of nineteenth century. It emerges from this study that the state of crisis of the teaching of literature is permanent and has branched into numerous conjectural forms. In the second part, we examine the theorical contributions of different fields of reference concerning the literary texts and their study as well the didactic answers to the problems which are posed by the study of the literary texts. In the third part, we focus on the "literary reading" in relation to the teaching/learning of writing and also with regard to the problematics such texts and history, texts and values which in extension of the "literay reading". In the fourth part, we are going to give a general survey of the current state of the theories of reference, as well as the present direction of the researches in didactic of the literature, including those currently appearing in the journal Pratiques. In conclusion, the notion of "competence" will be discursed so as to weigh its relevance to the teaching/learning of the "literary reading".

\section{INDEX}

Mots-clés : histoire de la didactique de la littérature, lecture littéraire, 40 années de recherches dans la revue Pratiques

Keywords : history of didactic of literature, literary reading, 40 years history of researchs in the review Pratiques

\section{AUTEUR}

\section{ANDRÉ PETITJEAN}

CREM (EA 3476), université de Lorraine 